Florida International University FIU Digital Commons

$11-19-2007$

\title{
Robust and High Current Cold Electron Source Based on Carbon Nanotube Field Emitters and Electron Multiplier Microchannel Plate
}

Raghunandan Seelaboyina

Florida International University

DOI: $10.25148 /$ etd.FI10022548

Follow this and additional works at: https://digitalcommons.fiu.edu/etd

\section{Recommended Citation}

Seelaboyina, Raghunandan, "Robust and High Current Cold Electron Source Based on Carbon Nanotube Field Emitters and Electron Multiplier Microchannel Plate" (2007). FIU Electronic Theses and Dissertations. 217.

https://digitalcommons.fiu.edu/etd/217 


\section{FLORIDA INTERNATIONAL UNIVERSITY}

Miami, Florida

ROBUST AND HIGH CURRENT COLD ELECTRON SOURCE BASED ON CARBON NANOTUBE FIELD EMITTERS

AND ELECTRON MULTIPLIER MICROCHANNEL PLATE

A dissertation submitted in partial fulfillment of the

requirements for the degree of

DOCTOR OF PHILOSOPHY

in

MECHANICAL ENGINEERING

by

Raghunandan Seelaboyina

2007 
To: Interim Dean Amir Mirmiran

College of Engineering and Computing

This dissertation, written by Raghunandan Seelaboyina, and entitled Robust and High Current Cold Electron Source Based on Carbon Nanotube Field Emitters and Electron Multiplier Microchannel Plate, having been approved in respect to style and intellectual content, is referred to you for judgment.

We have read this dissertation and recommend that it be approved.

Surendra K. Saxena

W. Kinzy Jones

Roberto R. Panepucci

Wonbong Choi, Major Professor

Date of Defense: November 19, 2007

The dissertation of Raghunandan Seelaboyina is approved.

Interim Dean Amir Mirmiran

College of Engineering and Computing

Dean George Walker

University Graduate School

Florida International University, 2007 


\section{ACKNOWLEDGMENTS}

This research work is a synergetic product of many minds to whom I would like to express my sincere gratitude.

I am extremely grateful for the inspiration, wisdom, insights, and constant encouragement of my major advisor Dr. Wonbong Choi. He has been a key force for this research work and it would not have been possible without his never-failing support, and guidance.

I would like to express my sincere gratitude to my committee members, Dr. Surendra K Saxena, Dr. W. Kinzy Jones and Dr. Roberto R Panepucci for their invaluable suggestions, assistance and attention in the completion of my Ph.D. work. I am very much thankful to them for providing me the resources and facilities during the course of this work. Their suggestions towards my research work helped me in solving several problems.

I sincerely acknowledge Dr. Jucheol Park and Dr. Dong Hun Kang of Samsung Advanced Institute of Technology for their assistance in TEM analysis of my samples. I sincerely acknowledge the assistance of Neal Ricks, Richard Castillo and Dr. Yanging Liu of Advanced Materials Research Institute, FIU for assisting me with sample preparation, sputtering, and electron microscopy work. I am also thankful to Dr. Andriy Durygin, of Center for Study of Matter at Extreme Conditions for his assistance with LabView programming and measurement circuitry.

I would like to acknowledge the financial support of Air Force Office of Scientific Research (AFOSR grant number-FA 9550-05-1-0232) for this work. 
I would like to thank all the former and present members of my research group Dr. Young Chul Choi, Dr. Do Hyun Kim, Dr. Somenath Roy, Harindra, Ved Verma, Jun Huang, Srinivasarao Bodepalli for their assistance and discussions which had helped me in solving various problems in course of this research work. I am thankful to Srinivas $\mathrm{R}$ Kulkarni for his assistance in the CAD drawings, Feng Zeng for his assistance with materials, punching machine and suggestions and Mechanical and Materials Department faculty and staff that had helped in numerous occasions and last but not the least to all my dear friends and Dr. Hexiong Yang.

I would like to express my deep sense of gratitude to my beloved parents, sister Neelu, brother Kranthi, fiancé Hema and family members for their constant love, insights, inspiration unwavering support and patience. 


\author{
ABSTRACT OF THE DISSERTATION \\ ROBUST AND HIGH CURRENT COLD ELECTRON SOURCE BASED ON \\ CARBON NANOTUBE FIELD EMITTERS AND \\ ELECTRON MULTIPLIER MICROCHANNEL PLATE
}

by

Raghunandan Seelaboyina

Florida International University, 2007

Miami, Florida

Professor Wonbong Choi, Major Professor

The aim of this research was to demonstrate a high current and stable field emission (FE) source based on carbon nanotubes (CNTs) and electron multiplier microchannel plate (MCP) and design efficient field emitters. In recent years various CNT based FE devices have been demonstrated including field emission displays, $\mathrm{x}$-ray source and many more. However to use CNTs as source in high powered microwave (HPM) devices higher and stable current in the range of few milli-amperes to amperes is required. To achieve such high current we developed a novel technique of introducing a MCP between CNT cathode and anode. MCP is an array of electron multipliers; it operates by avalanche multiplication of secondary electrons, which are generated when electrons strike channel walls of MCP. FE current from CNTs is enhanced due to avalanche multiplication of secondary electrons and in addition MCP also protects CNTs from irreversible damage during vacuum arcing.

Conventional MCP is not suitable for this purpose due to the lower secondary emission properties of their materials. To achieve higher and stable currents we have 
designed and fabricated a unique ceramic MCP consisting of high SEY materials. The MCP was fabricated utilizing optimum design parameters, which include channel dimensions and material properties obtained from charged particle optics (CPO) simulation. Child Langmuir law, which gives the optimum current density from an electron source, was taken into account during the system design and experiments. Each MCP channel consisted of $\mathrm{MgO}$ coated CNTs which was chosen from various material systems due to its very high SEY. With MCP inserted between CNT cathode and anode stable and higher emission current was achieved. It was $~ 25$ times higher than without MCP. A brighter emission image was also evidenced due to enhanced emission current. The obtained results are a significant technological advance and this research holds promise for electron source in new generation lightweight, efficient and compact microwave devices for telecommunications in satellites or space applications. As part of this work novel emitters consisting of multistage geometry with improved FE properties were was also developed. 
TABLE OF CONTENTS

CHAPTER

PAGE

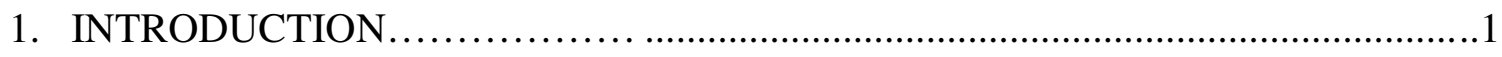

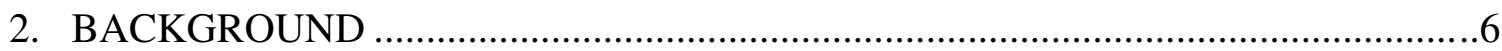

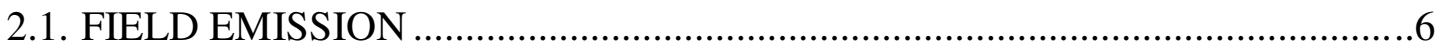

2.1.1. FOWLER-NORDHEIM (FN) THEORY ...........................................6

2.2. CARBON NANOTUBE (CNTS) FIELD EMITERS .................................... 10

2.3. TUNGSTEN OXIDE $\left(\mathrm{WO}_{\mathrm{X}}\right)$ MULTISTAGE FIELD EMITTERS ................... 14

2.4. ELECTRON MULTIPLIER MICROCHANNEL PLATE (MCP) .................... 18

2.5. SECONDARY ELECTRON EMISSION ................................................ 20

2.5.1.INSULATOR ELECTRON EMISSION AND SEMIEMPIRICAL

MODELS ................................................................................... 23

2.6. MICROCHANNEL PLATE SIMULATION ...............................................26

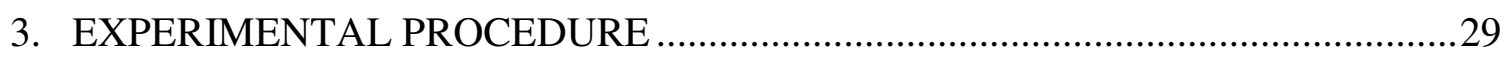

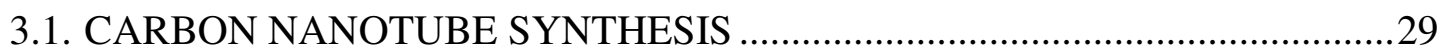

3.1.1. thin-MWNT SYNTHESIS ............................................................29

3.1.2. MWNT ARRAY TOWER STRUCTURE SYNTHESIS ........................31

3.1.3. TUNGSTEN OXIDE NANOWIRE MULTISTAGE FIELD EMITTERS............................................................................. 33

3.2. FIELD EMISSION MEASUREMENT EXPERIMENTAL PROCEDURE ......35

3.3. MICROCHANNEL PLATE FABRICATION ............................................... 37

3.3.1. GROWTH OF CARBON NANOTUBES ALONG THE MCP CHANNELS AND SURFACE ........................................................4 40

3.4. SECONDARY ELECTRON EMISSION (SEE) CHARECTERIZATION .......43

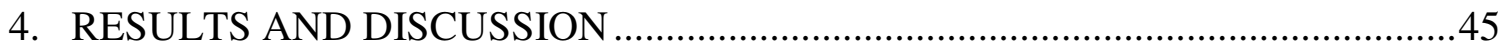

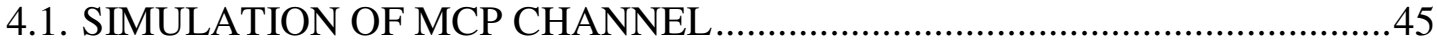

4.1.1. SIMULATION OF A STRAIGHT MCP CHANNEL ….......................47

4.1.2. SIMULATION OF A TILTED MCP CHANNEL ..................................49

4.1.3. SIMULATION OF A MCP CHANNEL WITH VARIOUS MATERIAL SYSTEMS ....................................................................52

4.2. SECONDARY ELECTRON EMISSION CHARECTERIZATION .................54

4.2.1. MgO-COATED CARBON NANOTUBES (CNTs) SECONDARY ELECTRON EMISSION ................................................................59

4.3. SYNTHESIS AND FIELD EMISION CHARECTERIZATION OF thin-

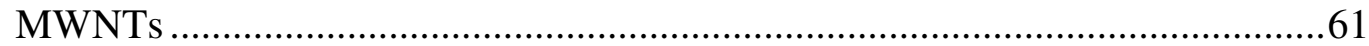

4.3.1. SYNTHESIS AND FIELD EMISSION CHARECTERIZATION OF TUNGSTEN OXIDE MULTISTAGE EMITTERS .................................6

4.3.2. SYNTHESIS AND FIELD EMISSION CHARECTERIZATION OF CNT MULTISTAGE PILLAR ARRAYS . .76 


\subsection{FABRICATION AND AMPLIFICATION CHARECTERIZATION OF A}

NEW MICROCHANNEL BASED ON HIGH SECONDARY YIELD

$\mathrm{MgO}-\mathrm{COVERD}$ CNT

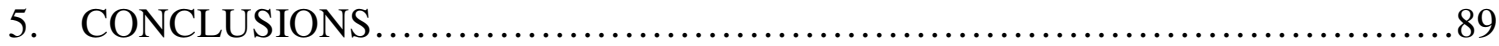

5.1 MICRO CHANNEL PLATE SIMULATION .............................. 89

5.2 SECONDARY EMISSION CHARACTERIZATION ..........................90

5.3 SYNTHESIS OF EFFICIENT FIELD EMITTERS..........................90

5.3 .1 thin-MWNTs..................................................... 90

5.3 .2 MULTISTAGE CNTs............................................. 91

5.3.3 MULTISTAGE TUNGSTEN OXIDE NANOWIRES ................... 91

5. 4 FABRICATION OF NOVEL HIGH SECONDARY EMISSION MCP..... 92

6. RECOMMENDATIONS FOR FUTURE WORK ............................93

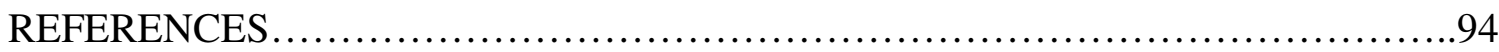

APPENDICES........................................................... 101

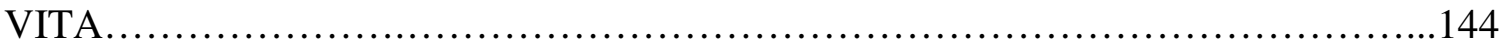




\section{LIST OF FIGURES}

FIGURE

PAGE

2..1 Potential energy $\mathrm{u}(\mathrm{x})$ of an electron in ev as a function of its distance $\mathrm{x}$ from the metal surface, is the image force potential, is the external applied potential, $u(x)$ is total potential, $\varphi$ is work function, up is potential energy of electron in the metal

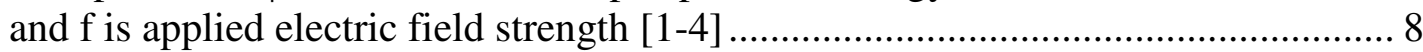

2.2 Tem images of various types of carbon nanotubes, SWNT (left), double wall CNT(middle), MWNT (right) [6]

2.3 Potential energy diagram for planar and microtip emitter (left), field enhancement at the emitter tip (right) [20]

2.4 Schematic of single-stage and multi-stage emitter geometry

2.5 Schematic of hemi-ellipsoid on a plane geometry of the electrochemically sharpened tungsten tip (geometrical/mathematical model), where $l$ is the semi-major axis length and $\rho$ is the semi-minor axis length (left) schematic of experimental configuration and a simple model of two-stage emitter, where 1 is the length and $r$ is radius of the nanowire. 1 is the length and $r$ is the radius of electrochemically sharpened tungsten tip (right)

2.6 Cross sectional image of mcp and structure of channel cross section (inset) (a) illustration of electron multiplication along the channel (b)

2.7 Electron beam interaction with a solid surface.

2.8 Total yield i.e. backscattered $(\eta)$ and secondary $(\delta)$ as a function of incident beam energy $(e)$

2.9 Dependence of the see yield $(\delta)$ on the primary electron beam energy, for different electron escape depth [56]....

2.10 (a) Zigzag modes in a non-tilted cylindrical channel for two primary electrons. the electric field (e) is along the channel axis, (b) a hopping mode in a tilted channel $\left(15^{\circ}\right)$ for two primary electrons. $E_{t}$ and $E_{n}$ are electric field components in the tangential and normal direction, respectively [47]

3.1 SEM images of (a) MWNT array tower structures synthesized on porous silicon (b) thin- MWNTs synthesized on $\mathrm{Nb} / \mathrm{SiO}_{2} /$ si substrates.

3.2 Schematic of porous silicon fabrication experimental setup 
3.3 Etching setup schematic (left) sem image as etched tungsten wire (middle) and tungsten oxide multistage i.e. nanowires on sharp tungsten tip (right)....

3.4 Field emission measurements circuit schematic, CNT (top) and $\mathrm{WO}_{\mathrm{x}}$ (bottom), please refer figure 2.5 in chapter for the meaning of various parameters

3.5 Field emission (FE) measurement system, vacuum chamber $\left(\sim 10^{-7}\right.$ torr $)$ and various measurement meters

3.6 Labview interface showing the program used to measured the I-V characteristics of the field emitters

3.7 (a) Aluminum oxide/alumina $\left(\mathrm{Al}_{2} \mathrm{O}_{3}\right)$ templates (green tape) with micron sized

(b) sintering cycle for alumina green body

3.8 Cross-sectional view of microchannel plate and its channel

3.9 Microchannel plate consisting of MgO-coated carbon nanotubes, smaller inset shows the uniform growth of nanotubes along the channel and larger inset shows the $\mathrm{MgO}$-coated nanotubes

3.10 Secondary electron emission measurement cell schematic

4.1 Trajectories of primary and secondary electrons for various aspect ratios mcp channels10, 20, 30, 40, 50 (left-right) 48

4.2 Secondary emission yields $(\delta)$ as a function of channel aspect ratio.

4.3 Trajectories of primary and secondary electrons for a tilted channel $\left(5^{\circ}\right)$

4.4 Secondary emission yields $(\delta)$ as a function of channel tilt .52

4.5 Various material system secondary emissive yields

4.6 SEM images of elecrophoretically deposited $\mathrm{MgO}$ from sol-gel solution (top) and dispersant on a flat metal surface

4.7 Secondary electron mission characterization of $\mathrm{MgO}$ prepared by sol-gel and suspensions

4.8 SEE yield of $\mathrm{MgO} / \mathrm{CNT}$ as a function of the relative kinetic energy of a primary electron. 
4.9 SEM images of thin-MWNTS with (a) $15 \mathrm{mg}$ and (b) $7.5 \mathrm{mg}$ of $\mathrm{Al}_{2} \mathrm{O}_{3}$ nanoparticles in catalyst solution (c) TEM image of thin-MWNTS (d) Raman spectrum of thin-MWNTs (laser excitation wavelength of $785 \mathrm{~nm}$ ) with Ar-Ne laser and inset radial breathing mode (RBM) peaks, numbers correspond to inner tube diameters.

4.10 (a) I-V plot of thin-MWNTs, (inset) FN plot (b) normalized emission current as a function of time measured at $400 \mathrm{~V}$ (inset) emission image.. ..................64

4.11 (a) Emission current vs. Applied voltage plot of thin- MWNTs in MCP biased condition and without MCP (inset FN plot \& emission image with well defined spots, $\mathrm{V}_{\mathrm{a}}=1500, \mathrm{~V}_{\mathrm{bot}}=100, \& \mathrm{~V}_{\text {top }}=500 \mathrm{~V}$ ) (b) schematic of measurement circuit. Distance between anode-cathode (right) \& distance between MCP-cathode (left) is 380

4.12 (a) SEM image of tungsten oxide nanowires grown on electrochemically etched tungsten tip (two-stage emitter geometry) (b) high magnification tem image of the $\mathrm{W}_{18} \mathrm{O}_{49}$ nanowires (monoclinic, $\mathrm{P} 2 / \mathrm{m}$, jcpds card number 84-1516), inset shows the two dimensional fourier transform pattern of the TEM image

4.13 (a) Field emission measurements i.e. Field vs. Current of tungsten oxide multistage (two-stage) field emitter and its corresponding FN plot shown in inset. R1: linear current voltage dependence, R2: slow current variation (saturation region), R3: rapid rise of voltage region, respectively. Field enhancement factor $(\beta)$ for these three regions $\sim 2900,19800$ and 3500 respectively (b) schematic of hemi-ellipsoid on a plane geometry of the electrochemically sharpened tungsten tip (geometrical/ mathematical model), where l' is the semi-major axis length and pis the semi-minor axis length (c) schematic of experimental configuration and a simple model of two-stage emitter, where 1 is the length and $\mathrm{r}$ is radius of the nanowire. $\mathrm{L}$ is the length and $\mathrm{r}$ is the radius of electrochemically sharpened tungsten tip.

4.14 (a) Field emission behavior of tungsten oxide multistage (two-stage) field emitter under various vacuum conditions i.e. $10^{-6}-10^{-3}$ torr (b) solid triangles indicate measurements made at $10^{-6}$ torr and solid stars indicate emission recovery measurement at $10^{-6}$ torr after measurements at $10^{-3}$ torr

4.15 Aligned carbon nanotube arrays grown on porous silicon .76

4.16 SEM images of (a) Vertically aligned/oriented MWNT arrays grown on porous silicon (b) plasma etched MWNTs (c) carbon nanotube multistage structure i.e. thin-MWNT and SWNT on top of MWNT (d) Schematic of the multistage structure and field emission measurement schematic 
4.17 HRTEM images of (a) MWNT and (b) thin-MWNT (c) Raman spectrum ( $\lambda: 514$ $\mathrm{nm}$ ) of multistage CNTs with Argon ion ( $\mathrm{Ar}+$ ) laser, inset radial breathing mode (RBM) peaks, inset numbers correspond to inner tube diameters of thin-MWNTs and SWNT.

4.18 Field emission plot i.e. emission current vs. applied field, for multistage and only MWNT arrays, turn-on field (Et) was $\sim 1.5$ times lower and emission current was $\sim 32$ times higher for multistage in comparison to only MWNT arrays, inset corresponding FN plot.

4.19 SEM image of (a) Ceramic $\left(\mathrm{Al}_{2} \mathrm{O}_{3}\right)$ microchannel plate (MCP) with $\mathrm{MgO}$-coated CNTs (b) crossection of MCP channel showing uniform layer of MgO-coated CNTs (c) magnified view of MgO-coated CNTs along the channel and (d) representative EDX spectrum of one of the several ponits taken along the channel with $\mathrm{MgO}$-coated CNTs showing the presence of $\mathrm{Mg}, \mathrm{O}, \mathrm{C}$ and

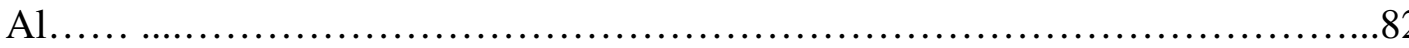

4.20 (a) HRTEM image of MgO-covered CNT, higher amount of $\mathrm{MgO}$ coating can be seen on side of the CNT from the image; which might be the nanotube surface facing away from the channel surface (b) high angle annular dark-field (HAADF) image of MgO-covered CNT (shown in figure 2a) acquired for 120 minutes (c) EDX spectrum i.e. line scan along the $\mathrm{MgO}$-covered CNT showing the presence of $\mathrm{MgO}$, higher amount of $\mathrm{MgO}$ coating on one side of nanotube can also be observed from the scan.............................................. 83

4.21 (a) Schematic of the CNT and MCP cold electron source (b) CNT emitters Field..84

4.22 (a) Field emission data (I-V) with and without MCP between CNT cathode and anode and only MCP (inset) showing the turn-on voltage (b) bright and focused emission with MCP over the CNT cathode. Increase in brightness is due to amplification of input current by secondary electron generation and well defined spots are due to focusing achieved from MCP channels (c) schematic of measurement circuit with and without MCP

4.23 Simulation results various materials systems considering them as the emissive layer along the channel that had the similar dimensions as the fabricated MCP in the present work. Among the material systems MgO-coated CNTs show the highest secondary yield. This shows that high multiplication achieved for the

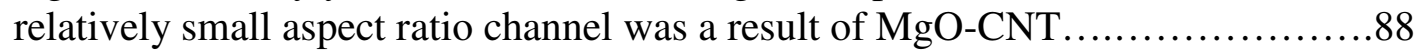




\section{LIST OF SYMBOLS}

j

$\mathrm{F}$

$\mathrm{D}$

$\mathrm{N}$

$N\left(E_{x}\right) \quad$ Number of electrons per second having the energies between $E_{x} \& E_{x}+d E_{x}$.

$\mathrm{E}_{\mathrm{x}} \quad$ Kinetic energy.

$\mathrm{p}_{\mathrm{x}} \quad$ Momentum component normal to the surface.

m Free electron rest mass.

$\mathrm{U}(\mathrm{x}) \quad$ Total potential.

$\Phi \quad$ Work function.

e Electron charge.

$\mathrm{U}_{\mathrm{p}} \quad$ Potential energy of electron.

$\mathrm{X}$

$v(\mathrm{y}) \quad$ Nordheim function or field dependent correction factor.

$\beta \quad$ Field enhancement factor.

i or I Current.

T Temperature.

L" Semi-major axis length.

$\rho \quad$ Semi-minor axis length.

L Length of tungsten emitter.

R Radius of tungsten emitter.

$l \quad$ Length of nanowire. 


\begin{tabular}{|c|c|}
\hline$r$ & Radius of nanowire. \\
\hline$i_{s}$ & Secondary current. \\
\hline$i_{p}$ & Primary current. \\
\hline$i_{t}$ & Specimen current. \\
\hline$\delta$ & Yield/Gain. \\
\hline $\mathrm{E}_{1}$ & First crossover energy. \\
\hline $\mathrm{E}_{2}$ & Second crossover energy. \\
\hline$\varepsilon$ & Energy required to generate a secondary electron. \\
\hline $\mathrm{B}$ & Probability for a secondary electron to reach the surface. \\
\hline $\mathrm{E}_{\mathrm{g}}$ & Band gap. \\
\hline $\mathrm{d}$ & Penetration depth. \\
\hline A & Primary absorption coefficient. \\
\hline$\alpha$ & Secondary absorption coefficient. \\
\hline$\rho_{\mathrm{m}}$ & Mass density. \\
\hline $\mathrm{E}_{\mathrm{p}}$ & Energy of primary electron. \\
\hline$f_{\text {mean }}$ & Maximum secondary electron yield. \\
\hline$a_{m}$ & Material constant. \\
\hline cs & Cosine angle of primary electron. \\
\hline $\mathrm{e}_{\max }$ & Maximum energy of primary electron. \\
\hline $\mathrm{E}_{1}$ & Local electric field at the emitter tip. \\
\hline $\mathrm{E}_{\mathrm{m}}$ & Macro electric field. \\
\hline $\mathrm{FN}$ & Fowler Nordheim. \\
\hline $\mathrm{b}$ & Slope of FN plot. \\
\hline
\end{tabular}


$d_{t} \quad$ Diameter of the nanotubes.

$\omega_{\text {RBM }} \quad$ Wavelength of RBM peaks. 


\section{INTRODUCTION}

The potential of utilizing carbon nanotubes (CNTs) field emission (FE) property has been an attractive feature for various devices [1-4]. To further extend this unique property as an electron source in high current applications such as microwave devices for communications, high total current $(\sim 1-10 \mathrm{~mA})$ and stability are required. This can be achieved by placing a secondary emission device such as microchannel (MCP) over the CNT emitters [5]. MCP is an array of microscopic channels that function as electron multipliers. They are utilized primarily as an amplification element in various image intensification devices including night vision, X-ray diagnostics systems and also in astronomy nuclear science and e-beam fusion studies and recently in high efficiency field emission display. MCP operates by avalanche multiplication of secondary electrons that are generated when incident electrons strike the channel walls. A voltage applied across the ends of the MCP channels creates a field accelerating the secondary electrons along the channel leading to avalanche multiplication [6-9]. They exist in two major modifications: discontinuous dynode and continuous dynode. In the former one the different dynodes are biased at higher potential than the previous one and the process of multiplication is achieved at successive dynodes. Finally the total current is measured at the anode. The later one consists of a continuous channel to which different bias is applied to inlet and outlet of the channel [6].

Conventional MCPs cannot be used for the purpose of amplifying field emission current as they are fabricated from lead silicate-glass which has relatively low secondary emission (SE). It restricts their amplifying capability which is typically in the range of few pico-amperes [6]. Thus they are inappropriate for amplifying high currents obtained 
from CNT and other field emitters. The gain i.e. ratio of output to input current of a MCP is a function of channels aspect ratio (length/diameter). To achieve a high gain the aspect ratio should be more than 40 and such channels are tough to fabricate [11-13]. Higher gain can also be achieved by applying high voltage; however, there is a practical limit for voltage that can be applied across a vacuum gap without breaking down [12]. It is governed by Child-Langmuir law, a relation involving current and applied voltage across the cathode and the anode. Application of higher voltage may also create ion feedback that can cause vacuum arcing. The most efficient method to achieve higher gain is to utilize a material with high secondary emission (SE) such as MgO covered CNTs. Recent reports have shown extremely high SE from $\mathrm{MgO}-\mathrm{CNT}$ system which is higher than any other known materials [14-15]. MgO, which is an insulator, has been shown to have relatively high secondary emission yield (SEY) (secondary/primary electron current). In insulators the lack of electron-electron interaction during the transport of the generated secondaries combined with their low electron affinity results in high SEY, which is explained by various theoretical considerations [16]. The lack of electron interaction leads to attenuation lengths $\sim 100 \mathrm{~nm}$ for secondary electrons in uncharged insulators and the low affinity permits them to escape easily into vacuum [16]. When CNTs are coated with $\mathrm{MgO}$, extremely high SEY value as high as 22000 [14] (dependent on various parameters) has been recently reported. It is extremely higher than independent SEY of CNT ( 1.2-2) or $\mathrm{MgO}(4-25)$ [13].

In recent years Govyadinov et al. [17] and Delendik et al. [18] have demonstrated a MCP based on anodic alumina (AAO). However, preparation of MCP from AAO and coating the channels with conductive and emissive layers is a laborious process. Yi et al. 
[9] has demonstrated alumina based MCP for high efficiency field emission display with $\mathrm{CuO}-\mathrm{SiO}_{2}$ material system. Its primary function was to improve the brightness of field emission display. Currently there are no available MCPs capable of amplifying input currents in $\mathrm{mA}-\mathrm{A}$ range. The motivation of this study was thus to develop a MCP capable of amplifying the input currents from field emitters in the range of few mA-A.

To the best of our knowledge this is the first study that demonstrates a MCP with MgO-CNT material system. In this work we demonstrate the fabrication of a new MCP consisting of $\mathrm{MgO}$ coated $\mathrm{CNT}$, show its current amplification when combined with CNT field emitters and provide a theoretical analysis to further improve the MCP performance.

In addition, this work also consisted of fabrication of efficient field emitter from carbon nanotubes and tungsten oxide nanowires. The emitters had a unique geometry which we termed as multistage. It consisted of smaller emitters on a larger one i.e. tungsten oxide nanowires on a sharp tungsten tip or thin-MWNTs/SWNT on MWNT. This geometry has shown to improve the field concentration at the emitter apex thus leading to lower threshold voltage and higher emission current. Apart from superior field emission properties the oxide emitters had a relatively stable emission current in various vacuum level i.e. $10^{-3}-10^{7}$ Torr. Emission of conventional metallic emitters is degraded irreversibly in low vacuum and oxygen environments because of enhanced surface reactions such as ion back sputtering, physical etching and so on [19-21]. Ion back sputtering and physical etching especially at the emitter tip surface degrades the local geometry and can lead to irreversible damage of the emitters. Oxide materials are chemically inert, robust and have relatively high melting temperature which leads to their stability in oxygen and low vacuum conditions i.e. $10^{-4}$ to $10^{-5}$ Torr. So, field emitters 
based on oxide materials have been reported as alternative candidates for stable field emission in low vacuum conditions [22-23].

Among the oxide emitters, tungsten oxide is widely used in various applications such as high pressure discharge lamps, electron guns and scanning tunneling microscopy tips. Tungsten oxide has also been demonstrated to be suitable for various other applications such as electrochromic, optochromic and gasochromic coatings for smart windows, information display and various sensors [24-25]. Tungsten oxide is an n-type semiconductor with a work function in the range of 5.59-5.7 eV [26-28] which makes it attractive for the stated applications. Since the first report on tungsten oxide by Fumio et al. in 1971 [29], several other researchers have synthesized tungsten oxide nanowires [3034]. Some of these synthesis methods include thermal treatment of tungsten films [30], infrared irradiation heating of tungsten foils [31] and thermal oxidation approach [34]. Recently Zhou et al. has reported the synthesis of tungsten oxide nano-tip arrays by a two-step, high temperature, catalyst-free, physical vapor deposition [32]. Though field emission properties of tungsten oxide nanowires have been studied, to the best of our knowledge there are no reports on multistage field enhancement effect and their emission performance in various vacuum conditions. The experimental results of the multistage field enhancement effect of these tungsten oxide emitters were also validated from our theoretical calculations. The nanotube multistage emitter tower arrays were grown on porous silicon nano-template which assisted in achieving vertical alignment. These well spaced emitters were designed to minimize the field screening effect thus improving the emission current. To the best of our knowledge this was also the first work in nanotube multistage emitters. 
This dissertation work is structured as follows

- Chapter 1: Introduction.

- Chapter 2: This chapter provides the definitions, theory, and literature review, related to field emission, CNT field emitters, tungsten oxide $\left(\mathrm{WO}_{\mathrm{x}}\right)$ multistage field emitters, electron multiplier microchannel plate (MCP), secondary electron emission (SEE) of insulators and MCP simulation. The information presented in this chapter will be used in later chapters for interpretation and analysis of the experimental data.

- Chapter 3: Experimental procedure.

- Chapter 4: This chapter begins with section 4.1, which outlines the simulation of a microchannel plate channel with various aspect ratios, tilt and material parameters. Section 4.2 outlines the characterization secondary electron emission of various materials used in this work. Section 4.3 outlines the synthesis and field emission characterization of single stage thin multiwall carbon nanotube and the multi-stage tungsten oxide and nanotube field emitters. Finally section 4.5 outlines the fabrication of the novel microchannel plate with high secondary yield materials and the amplification of field emission current from nanotube emitters through it.

- Chapter 5: Conclusion and recommendations for future work.

- Appendix includes various experimental procedures, code and results of MCP simulation, Lab View block diagrams and technical papers published in peerreviewed journals and conference proceedings, during the course of this dissertation work are presented. 


\section{BACKGROUND}

This chapter provides the definitions, theory, and literature review, related to field emission, carbon nanotube $(\mathrm{CNTs})$ field emitters, tungsten oxide $\left(\mathrm{WO}_{\mathrm{x}}\right)$ multistage field emitters, electron multiplier microchannel plate (MCP), secondary electron emission (SEE) of insulators and MCP simulation. The information presented in this chapter will be used in later chapters for interpretation and analysis of the experimental data.

\subsection{FIELD EMISSION}

Field emission (FE) is an electron emission process in which electrons tunnel from a deformed potential barrier of a condensed phase i.e. metal, insulator or semiconductor under the application of high electric fields $10^{7}-10^{8} \mathrm{~V} / \mathrm{cm}$, it is due to quantummechanical effects. In order to produce such high field using reasonable potentials the emitter is usually formed into a tip with the apex radius in the range of few angstroms ( $\AA$ ) to several microns $(\mu \mathrm{m})$. The high electric field narrows the potential barrier at the metalvacuum interface sufficiently for the electrons to have a significant probability for tunneling from the metal into vacuum [35-38].

\subsubsection{FOWLER-NORDHEIM (FN) THEORY}

This section provides a brief description of FE process and the derivation of FowlerNordheim (FN) equation. Quantitative description of the FE process is given by FN theory [39] which gives a relation between current density $(j)$ as function of applied electric field $(F)$. For this the probability of electron to tunnel through the potential barrier has to be determined which depends on barrier transparency (D) and the total 
number of electrons $(N)$ incident on the barrier from within the metal. Following are the assumptions made for deriving the $\mathrm{FN}$ equation

1. Temperature of the metal is $0{ }^{\circ} \mathrm{K}$.

2. Surface is assumed to be smooth and plane, i.e. one dimensional problem is considered. It is assumed because in most cases the thickness of the potential barrier in the applied field range of $10^{7}-10^{8} \mathrm{~V} / \mathrm{cm}$ is several orders less than the emitter radius. Thus external field can be taken to be uniform along the surface.

3. Free electron approximation inside the metal with Fermi-Dirac statistics.

4. Potential with in the metal is considered constant. Potential barrier close to the surface in vacuum consists of an image-force potential $\left(-e^{2} / 4 x\right.$ is the Coulomb attraction towards the surface of an electron outside due to its induced charge in the metal) and potential due to applied electric field $-e F x$.

Under the above considerations current density is given by the equation,

$$
j=e \int_{0}^{\infty} n\left(E_{x}\right) D\left(E_{x}, F\right) d E_{x}
$$

Where $e$ is the electron charge, $n\left(E_{x}\right)$ is the number of electrons per second having the energies between $E_{x}$ and $E_{x}+d E_{x}$, incident on the barrier with a area of one square $\mathrm{cm}$ within the metal, $E_{x}=p_{x}^{2} / 2 m$ is the part of electron kinetic energy carried by the momentum component $p_{x}$ normal to the surface, $m$ is the free electron rest mass, and $F$ is the applied electric field. The barrier transparency is calculated using the WentzelKramers-Brillouin (WKB) approximation which is a semi-classical method. The potential function for an applied electric field is given by (fig 2.1) [35-38]. 


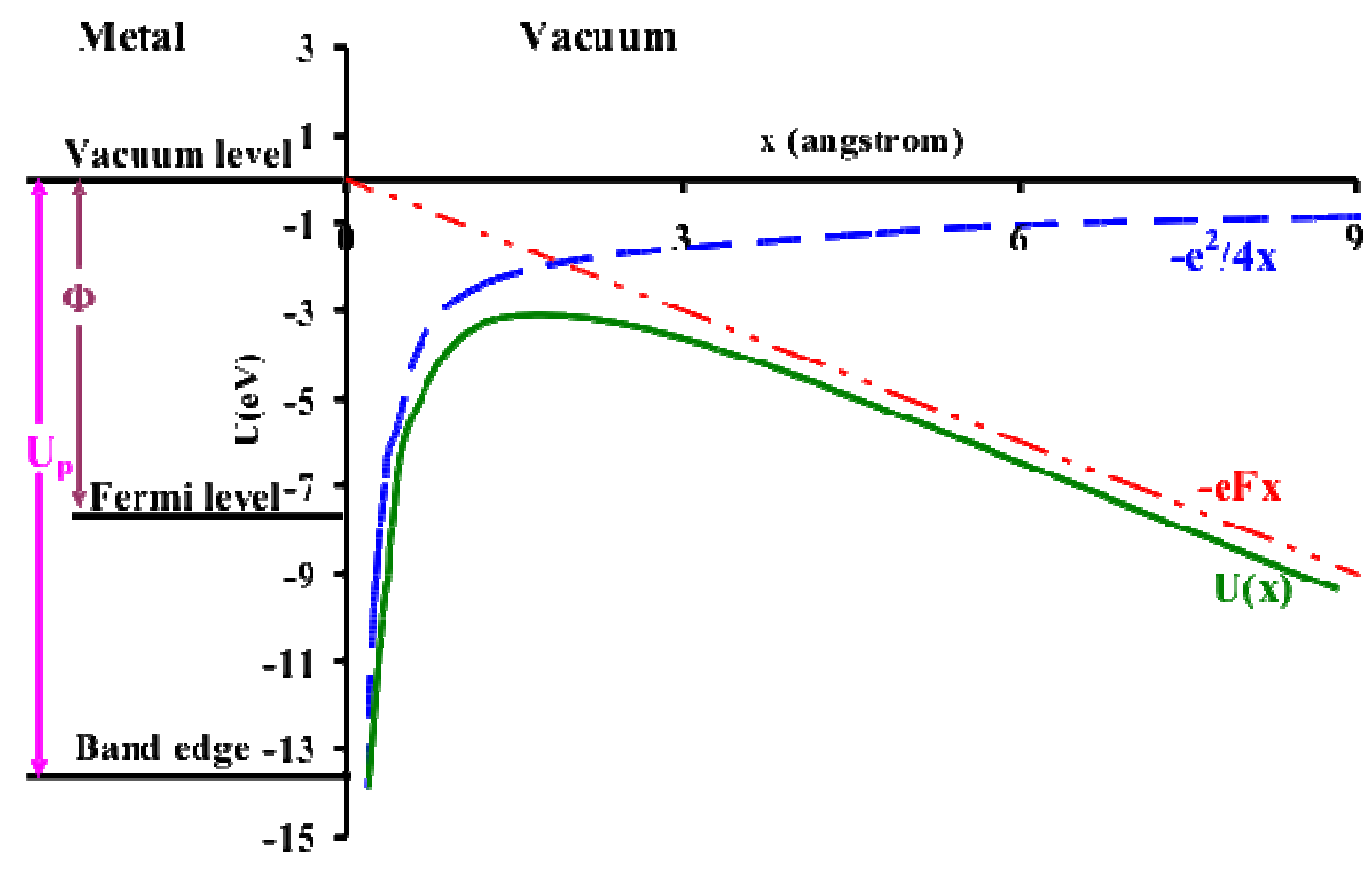

Figure 2. 1 Potential energy $\mathrm{U}(\mathrm{x})$ of an electron in $\mathrm{eV}$ as a function of its distance $\mathrm{x}$ from the metal surface, $-\mathrm{e}^{2} / 4 \mathrm{x}$ is the image force potential, $-\mathrm{eFx}$ is the external applied potential, $\mathrm{U}(\mathrm{x})$ is total potential, $\Phi$ is work function, $\mathrm{Up}$ is potential energy of electron in the metal and $\mathrm{F}$ is applied electric field strength [35-38].

$$
U(x)=\left[-\frac{e^{2}}{4 x}\right]-e F x
$$

For such potential barrier transparency is given by

$$
D\left(E_{x}, F\right)=\exp \left[-\frac{8 \Pi(2 m)}{3 h e}^{1 / 2}\right]{\frac{\left|E_{x}\right|^{3 / 2}}{F}}^{3 / 2} \vartheta(y)
$$

Where $\vartheta(y)$ is the Nordheim function or field dependent correction factor 


$$
\begin{array}{r}
\vartheta(y)=0.95-y^{2} \text { or } \cos \left(\frac{1}{2} \Pi y\right) \\
y=\frac{\left(e^{3} F\right)^{1 / 2}}{\phi}=\frac{3.79 \times 10^{-4} F^{1 / 2}}{\phi}
\end{array}
$$

Using equation (2.3) the field emission current density $(j)$ at $T=0$ is given by the classical FN formula,

$$
j=1.54 \times 10^{-6} \frac{F^{2}}{t^{2}(y) \phi}\left[-6.83 \times 10^{7} \frac{\phi^{2 / 3}}{F} \vartheta(y)\right]
$$

It is customary to express the field at the tip by $F=\beta V$ where $\beta$ is the field enhancement factor in $\mathrm{cm}^{-1}$, the field emission current is given by the classical FN formula,

$$
i=1.54 \times 10^{-6} \frac{(\beta V)^{2}}{t^{2}(y) \phi} \exp \left[-6.83 \times 10^{7} \frac{\phi^{3 / 2}}{\beta V} \vartheta(y)\right]
$$

Where $t^{2}(y)=\vartheta(y)-(2 y / 3)(d \vartheta(y) / d y) \cong 1, \phi$ is in $\mathrm{eV}, F$ in $V / \mathrm{cm}$ and $j$ in $A / \mathrm{cm}^{2}$. The FN equation (2.7) can be rewritten as,

$$
\ln \left(\frac{i}{V^{2}}\right)=\ln \left(1.54 \times 10^{-6} \frac{\beta^{2}}{\phi}\right)-6.87 \times 10^{7} \frac{\phi^{3 / 2}}{\beta V} \vartheta(y)
$$

Thus by plotting emission current in the form of $\ln \left(i / V^{2}\right) v s .1 / V$, a straight line should be obtained. From the plot $\beta$ can be determined using the slope which is proportional to $\phi^{3 / 2} \vartheta(y) / \beta$ (if value of $\phi$ is known) and emission area can be determined from intercept $\beta^{2} / \phi[35-38]$. These formulas would be used in the later chapters to analyze the data obtained from carbon nanotube and tungsten oxide field emitters. 


\subsection{CARBON NANOTUBE (CNTs) FIELD EMITTERS}

This section provides a brief description of CNTs, their uniqueness as field emitters, fabrication/synthesis methods and their various FE applications. Carbon nanotubes consists of either one cylindrical graphene sheet, termed single-wall nanotube (SWNT) or several nested cylinders with an interlayer spacing of $0.34-0.36 \mathrm{~nm}$, termed multi-wall carbon nanotube (MWNT). SWNTs and MWNTs lengths are usually well over $1 \mu \mathrm{m}$ with diameters ranging from $1 \mathrm{~nm}$ for SWNTs to $\sim 30 \mathrm{~nm}$ for MWNTs. TEM images of various CNTs is shown in figure 2.2 [40] Nanotubes are usually closed at both ends by fullerenelike half spheres that contain both pentagons and hexagons [41]. They have been envisioned as one of the promising materials for field emitters, because of their high aspect ratio $(\sim 1000)$ and atomically sharp apex, which enhances local field and lowers threshold field for electron emission $(\sim 0.5-1 \mathrm{~V} / \mu \mathrm{m})$ [42]. They also possess high electrical and thermal conductivity and high chemical and temperature stability all these unique properties make CNT a robust emitter with high emission stability [41]. FE from

CNTs was first reported for an individual multiwall carbon nanotube (MWNT) by Rinzler et al [42]. In later years the mechanism of electron emission from CNTs was confirmed to be FE process [43]. It has been well documented that CNTs have better FE properties than other sharp metal based emitters (table 1). 


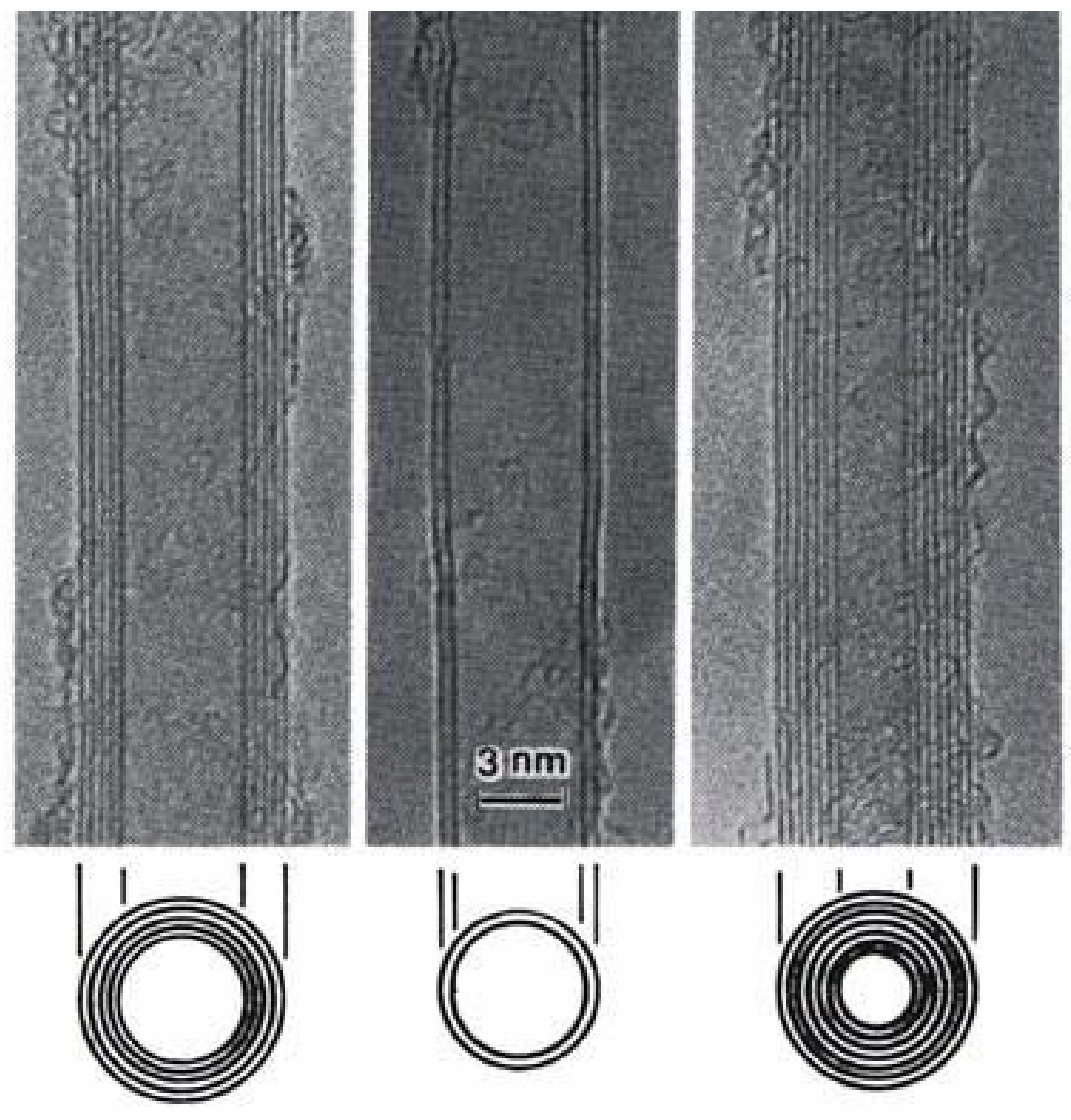

Figure 2. 2 TEM images of various types of carbon nanotubes, MWNT (left), Double wall CNT (middle), MWNT (right) [40].

Some of the drawbacks with metal based field emitters are high current fluctuations, high fabrication cost and limited life time owing to field-induced sharpening and ion sputtering, which leads to destruction of the emitters. CNT is robust and highly crystalline covalent structure which is less susceptible to surface migration of carbon atoms because of their strong $\mathrm{C}-\mathrm{C}$ covalent bonding [44]. A single $\mathrm{CNT}$ is capable of emitting $\sim 1-2 \mu \mathrm{A}(\mathrm{SWNT})$ or $\sim 3-5 \mu \mathrm{A}(\mathrm{MWNT})$ before saturating [45]. Saturation has been attributed either, to the presence of adsorbates at the apex of nanotube which enhance emission at low fields and are removed at high fields, or to the presence of 
resistance in series with the emitter i.e. nanotube/substrate electrical contact and internal resistance of CNT. However rapid thermal annealing (RTA) of the CNTs at $800^{\circ} \mathrm{C}$ has shown an increase in emission current by more than one order, due to improved crystallinity of the nanotube walls and improved nanotube/substrate contact [45].

Table 2.1 Properties of various field emitters [46].

Threshold electric field required for a $10 \mathrm{~mA} / \mathrm{cm}^{2}$ emission current density for different materials

\begin{tabular}{|c|c|c|c|c|c|}
\hline Cathode material & \multicolumn{5}{|c|}{$\begin{array}{l}\text { Threshold field }(\mathrm{V} / \mu \mathrm{m}) \text { for a } \\
\text { current density of } 10 \mathrm{~mA} / \mathrm{cm}^{2}\end{array}$} \\
\hline Mo tips & \multicolumn{5}{|c|}{$50-100$} \\
\hline Si tips & \multicolumn{5}{|c|}{$50-100$} \\
\hline p-type diamond & \multicolumn{5}{|c|}{160} \\
\hline Defective CVD diamond & \multicolumn{5}{|c|}{$30-120$} \\
\hline Amorphic diamond & \multicolumn{5}{|c|}{$20-40$} \\
\hline Cesium-coated diamond & \multicolumn{5}{|c|}{$20-30$} \\
\hline Graphite powders & \multicolumn{5}{|c|}{$10-20$} \\
\hline Nano-diamond & \multirow{2}{*}{\multicolumn{5}{|c|}{$\begin{array}{l}3-5\left(\text { unstable }>30 \mathrm{~mA} / \mathrm{cm}^{2}\right) \\
1-2\left(\text { stable }>4000 \mathrm{~mA} / \mathrm{cm}^{2}\right)\end{array}$}} \\
\hline Assorted carbon nanotubes & & & & & \\
\hline \multicolumn{6}{|c|}{ Effect of bundle length on the field emission properties of SWNTs } \\
\hline Average length $(\mu \mathrm{m})$ & $>10$ & $>10$ & 4.0 & 1.5 & 0.5 \\
\hline Field enhancement factor $\beta$ & 2423 & 2345 & 1021 & 623 & 602 \\
\hline Field for $10 \mu \mathrm{A} / \mathrm{cm}^{2}(\mathrm{~V} / \mu \mathrm{m})$ & 1.2 & 0.9 & 2.0 & 3.6 & 4.0 \\
\hline
\end{tabular}

Apart from these unique properties, vertically aligned CNTs (Fig.1a) and tower array structures that are vital for vacuum microelectronic applications have been demonstrated [47-49]. The vertically oriented high aspect ratio CNT structures will provide high field enhancement and low threshold voltage for electron emission. CNT based cathode operation depends on several parameters including; type of CNTs i.e. SWNT or MWNT, 
aspect ratio, orientation, uniformity, distance and density. CNTs have been synthesized by using various methods those include laser ablation, arc-discharge, microwave, ultrasonication and chemical vapor deposition (CVD). CVD is mostly used as it can produce large quantities of CNTs, which are obtained when a hydrocarbon feedstock gas is heated to $600-1000^{\circ} \mathrm{C}$ in the presence of a transition metal catalyst. Vertically aligned CNTs synthesis has also been demonstrated by application of electric field [50-52], plasma enhanced CVD (PECVD) and nano template assisted thermal CVD [48].

Ever since the first report on CNT FE there have been several reports on various devices. Some of the most promising ones are field emission displays (FED) [50], electron guns for next generation scanning electron microscopes (SEM) and transmission electron microscopes (TEM) [52], backlight for liquid crystal displays (LCD) [53-54], miniature X-ray $[3,55]$ and source for high powered microwave (HPM) devices [4]. Although there has been significant advancement in CNT based FE devices, there still remain some obstacles in realizing the devices. One of those issues achieving a moderately stable and high emission current. In this work we have utilized an electron multiplier microchannel plate to enhance the FE current from the emitters. MCP has been recently used in FED to improve the picture quality [9]; however there are no reports on using a MCP with CNT field emitters for enhancing the FE current. In the later chapters the emitter fabrication/synthesis, characterization, emission stability measurements and a modestly reliable and robust method for emission enhancement with an electron multiplier microchannel plate (MCP) will be discussed. 


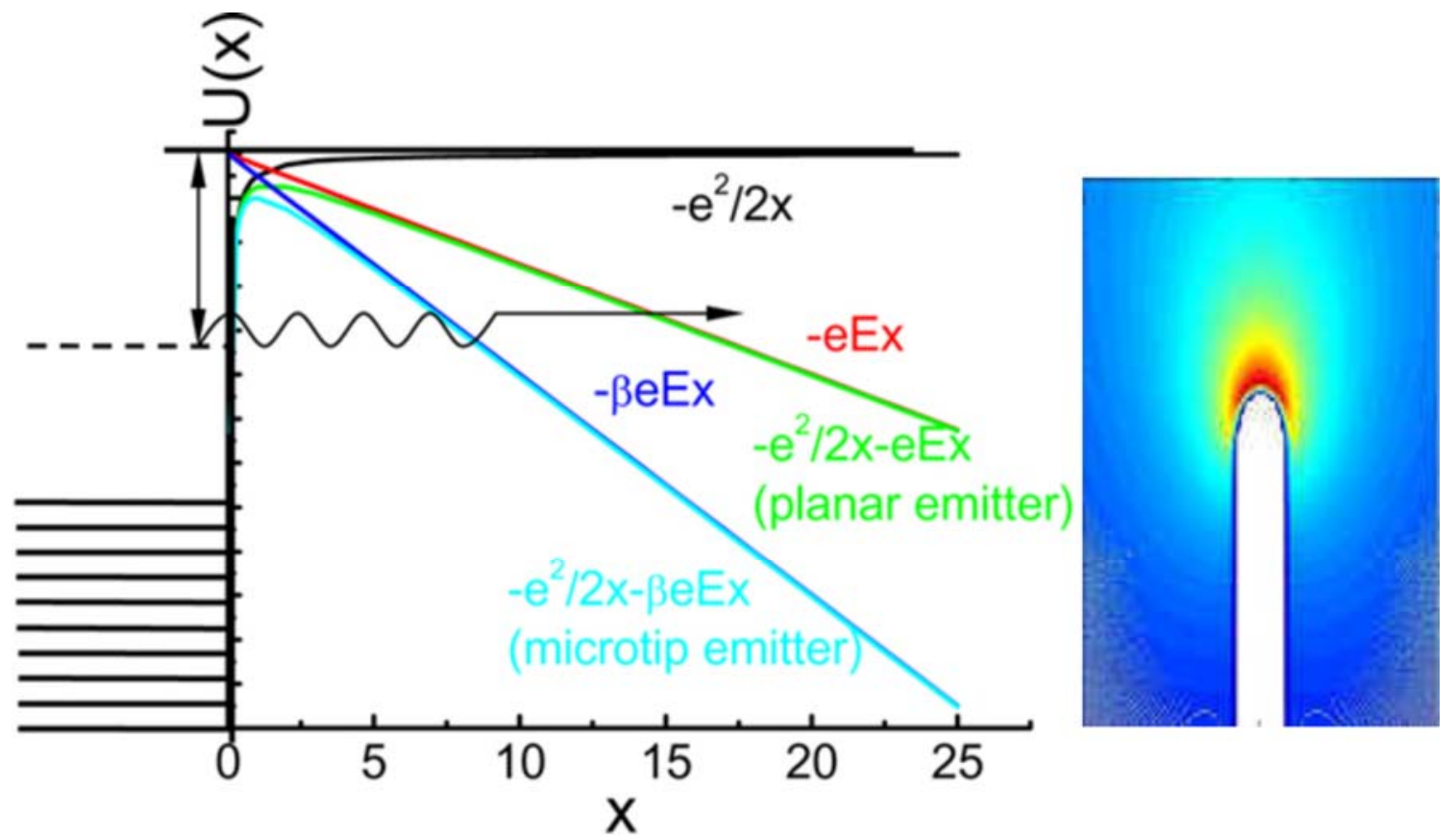

Figure 2. 3 Potential energy diagram for planar and microtip emitter (left), field enhancement at the emitter tip (right) [20].

\subsection{TUNGSTEN OXIDE (WOX $)$ MULTISTAGE FIELD EMITTERS}

This section provides a brief description of oxide based multistage emitters and their advantage. To date field emitters based on various nanomaterials have been considered for several vacuum microelectronic applications. These emitters utilize local field enhancement at the apex of each nanoscale protrusion or micro-tip to lower the threshold voltage for field emission. They have been demonstrated in various applications such as field emission displays, microwave sources and many more. However their emission property is degraded irreversibly in low vacuum and oxygen environments because of enhanced surface reactions such as ion back sputtering, physical etching and so on [19- 
21]. Ion back sputtering and physical etching especially at the emitter tip surface degrades the local geometry and can lead to irreversible damage of the emitters.

Oxide materials are chemically inert, robust and have relatively high melting temperature which leads to their stability in oxygen and low vacuum conditions i.e. $10^{-4}$ to $10^{-5}$ Torr. So, field emitters based on oxide materials have been reported as alternative candidates for stable field emission in low vacuum conditions [22-23]. Among the oxide emitters, tungsten oxide is widely used in various applications such as high pressure discharge lamps, electron guns and scanning tunneling microscopy tips. Tungsten oxide has also been demonstrated to be suitable for various other applications such as electrochromic, optochromic and gasochromic coatings for smart windows, information display and various sensors [24-25].

Tungsten oxide is an n-type semiconductor with a work function in the range of 5.59$5.7 \mathrm{eV}$ [26-28] which makes it attractive for the stated applications. Since the first report on tungsten oxide by Fumio et al. in 1971 [29], several other researchers have synthesized tungsten oxide nanowires [30-33]. Some of these synthesis methods include thermal treatment of tungsten films [30], infrared irradiation heating of tungsten foils [31] and thermal oxidation approach [32]. Recently Zhou et al. has reported the synthesis of tungsten oxide nano-tip arrays by a two-step, high temperature, catalyst-free, physical vapor deposition [34]. Though field emission properties of tungsten oxide nanowires have been studied, this is the first study on multistage geometry and the emission performance of such emitters in various vacuum conditions. A multistage emitter is defined as a nano-protrusion such as nanowire or nanotube on top of sharp tip, as seen in 
figure2.4. The multistage emitter geometry will lead to high field concentration thus high field enhancement and consequently high field emission.

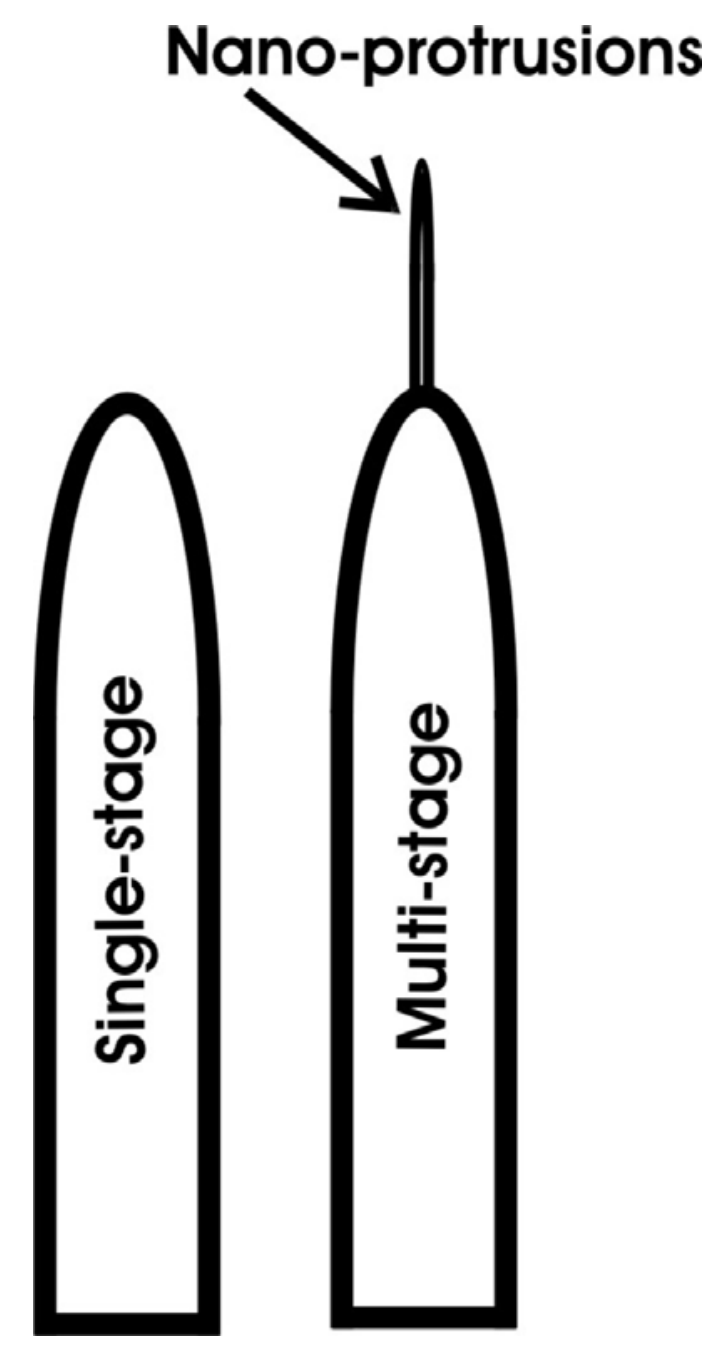

Figure 2. 4 Schematic of single-stage and multi-stage emitter geometry.

Multistage effect on field enhancement can be determined from the field enhancement factor $(\beta)$, which tells how much the applied electric field is enhanced at the tip (figure 2.3). The $\beta$ value of sharp tungsten tip without any nanowires on top of it can be calculated considering a hemi-ellipsoid on a plane geometry [56], which is given by 


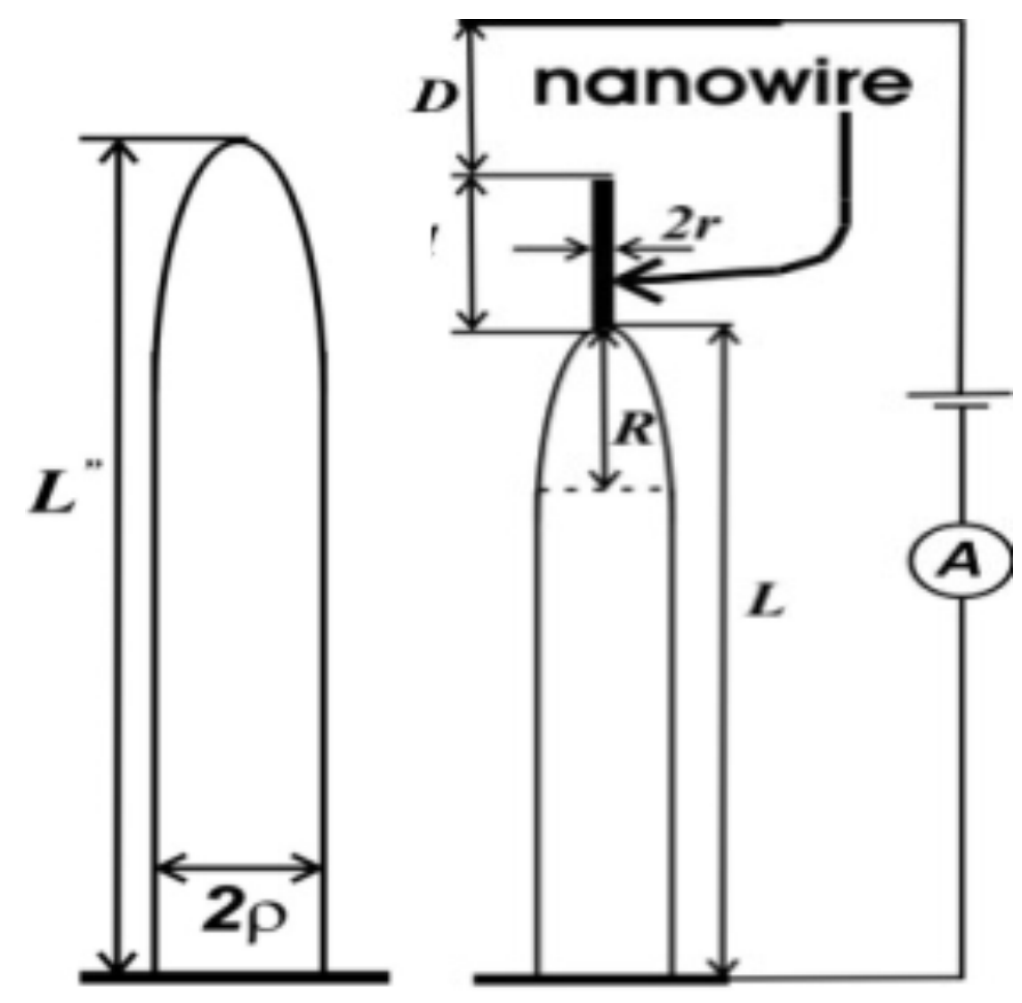

Figure 2. 5 Schematic of hemi-ellipsoid on a plane geometry of the electrochemically sharpened tungsten tip (geometrical/mathematical model), where $L^{\prime \prime}$ is the semi-major axis length and $\rho$ is the semi-minor axis length (left) Schematic of experimental configuration and a simple model of two-stage emitter, where $l$ is the length and $r$ is radius of the nanowire. $L$ is the length and $\mathrm{R}$ is the radius of electrochemically sharpened tungsten tip (right).

$$
\begin{gathered}
\beta=\zeta^{3} /[\{v \ln (v+\zeta)\}-\zeta] \\
\zeta=\left(v^{2}-1\right)^{1 / 2} \\
v=L / \rho
\end{gathered}
$$


Where $L$ is the semi-major axis length and $\rho$ is the semi-minor axis length (figure 2.5). $\beta$ for a multistage field emitter i.e. tungsten oxide nanowires on tungsten tip can be calculated using equation 2.32

$$
\beta_{t o t}=\beta_{n w} \beta_{t i p}=\frac{l}{(l+D)}\left(1+\frac{D}{r}\right) \frac{h}{(h+D)}\left(1+\frac{D}{R^{\prime}}\right)
$$

Where $l, r$ are the length and radius of the nanowire, while $L$ is the length, $R$ is the radius

of electrochemically sharpened tungsten tip and $\mathrm{D}$ is the inter-electrode distance. $R^{\prime}=R+l$ and $h=L+l$ are effective radius and length of the emitter i.e. nanowire and electrochemically sharpened tungsten tip [57]. In the later chapters these formulas would be used to analyze the experimental data and also the fabrication/synthesis method of the emitter and its emission characterization in various vacuum conditions will be discussed.

\subsection{ELECTRON MULTIPLIER MICROCHANNEL PLATE (MCP)}

This section provides a brief description on currently available MCP, novelty of our ceramic MCP, and its application to enhance FE current from field emitters. A microchannel plate is an array of miniature electron multipliers (figure 2.6). Typical channel diameters are in the range of 10-500 microns with an aspect ratio (length/diameter) of 40-100. Channel axes are normal or biased at an angle [6]. In the later chapter the affect of aspect ratio and channel tilt/angle on electron multiplication or total yield would be analyzed using charged particle optics (CPO). MCP was originally developed as an amplification element for image intensification devices, later on it has been utilized in several applications which including high efficiency field emission displays and many pulse counting applications [6]. At present, the main material for 
manufacturing MCP is lead-silicate glass, which has a limited gain due to the low secondary emissive yield [8-9]. High gain from microchannel plate can be obtained by using high secondary electron yield materials such as $\mathrm{MgO}$ and $\mathrm{SiO}_{2}$, increasing aspect ratio, applying high voltage [12]. In this work a novel ceramic MCP has been designed and fabricated which is capable of achieving high secondary emissive yield. The design of an individual channel i.e. an electron multiplier is as shown in figure 2.6a inset. In a MCP, each channel acts as a continuous or discontinuous dynode that supports an avalanche multiplication of electrons when bias potential is applied across its length (figure 2.5b). A high secondary emission can be achieved by using $\mathrm{MgO}, \mathrm{SiO}_{2}$ and other insulators as secondary emissive layer. Function of the resistive/metallic layer is to replenish electrons to SEE layer and also to apply a potential so as to accelerate the generated secondary electrons along the channel. The concept of secondary electron emission and the theory of secondary electron emission from insulators are provided in section 2.5. In the later chapters the design and fabrication of MCP will be discussed.

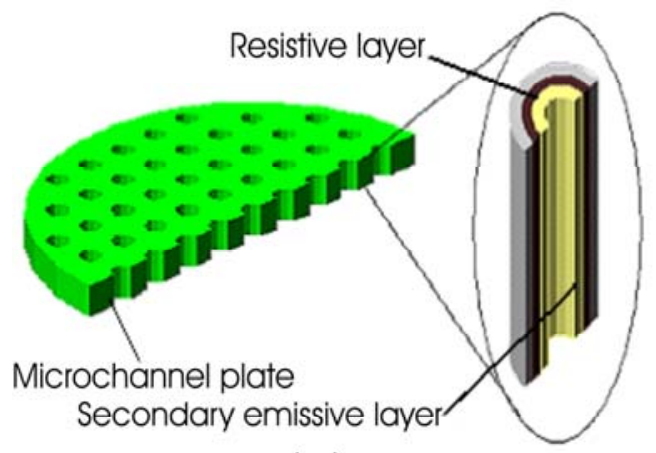

(a)

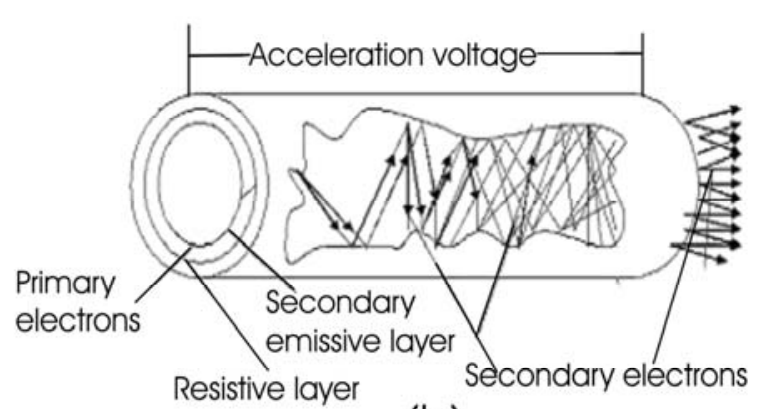

(b)

Figure 2. 6 Cross sectional image of MCP and structure of channel cross section (inset)

(a) Illustration of electron multiplication along the channel (b). 


\subsection{SECONDARY ELECTRON EMISSION}

This section provides a brief description of secondary electron emission. When electrically charged beam of particles which can be electrons, protons, or ions with sufficient kinetic energy hit the surface of a solid, the latter emits various kinds of electrons and X-rays which are shown in figure 2.7. Secondary electrons (SEs) are those which have a kinetic energy of $50 \mathrm{eV}$ or less, they are the basis of the most widely used imaging mode in the scanning electron microscope (SEM). They also play an important role in other areas which include particle accelerators and high efficiency field emission displays $[6,8]$.

The phenomenon of secondary electron emission was first discovered by Austin and Starke [59], during their study of cathode rays reflection from a metal surface. They observed that the metal target was emitting more number of electrons than it received. This was proof that primary electrons (bombarding electrons) liberated additional ones from the material itself [59]. Later on the phenomenon was thoroughly studied by Lenard and several other researchers for various metals, semiconductors and insulators. Also a database consisting of secondary emissive yield profiles for incident electron energies up to $50 \mathrm{keV}$, covering 51 elements and 42 compounds, and representing over 80 years of published data from several researchers has been compiled by Joy et al $[58,60]$. 


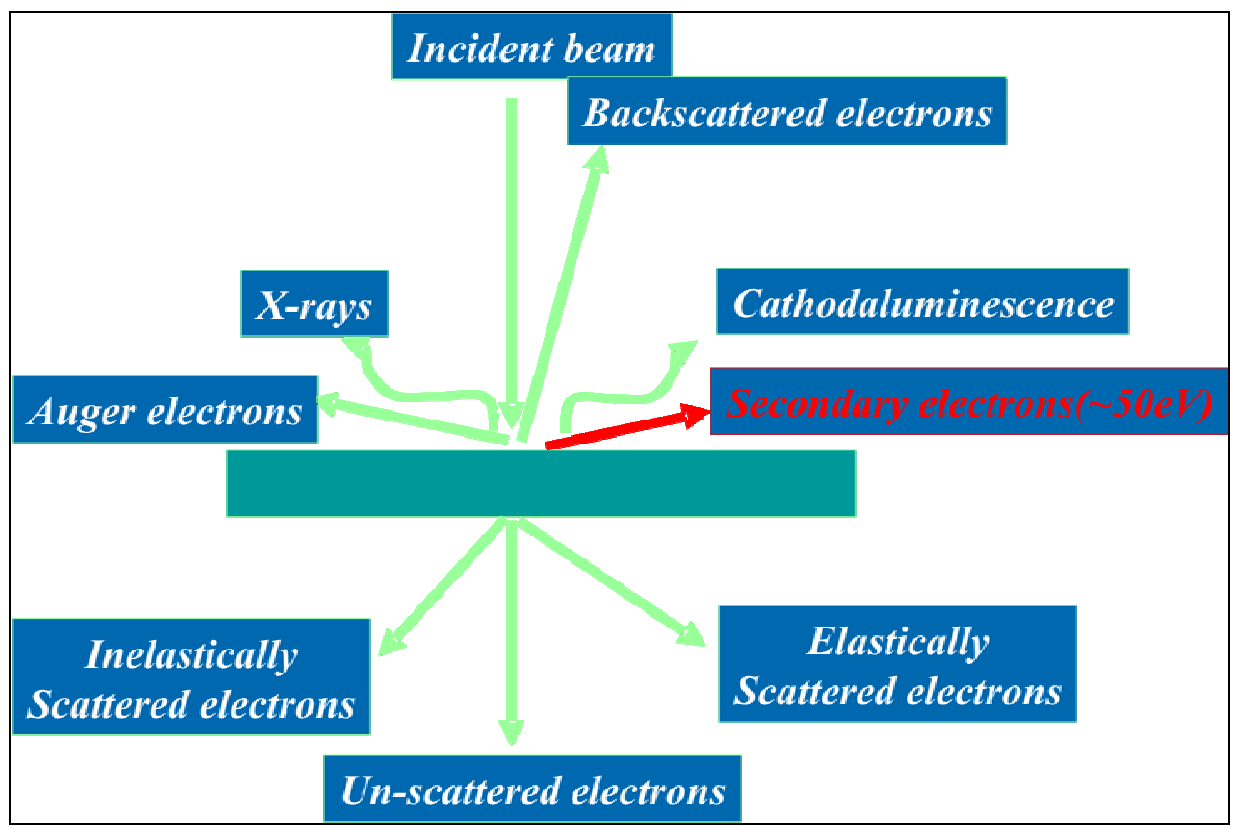

Figure 2. 7 Electron beam interaction with a solid surface.

Parameter describing the secondary electron emission (SEE) is the yield $(\delta)$ which is defined as the ratio of secondary electron $\left(i_{s}\right)$ to primary electron $\left(i_{p}\right)$ current given by equation 2.9. Variation of $\delta$ with the incident electron beam energy (figure 2.8) is the information that is obtained in the study of secondary electron emission using various methods [61-63]. $E_{1}$ and $E_{2}$ are the incident beam energies, usually known as first and second crossover energies, where total yield is greater than " 1 ", usually $\mathrm{E}_{2}$ is the range of 1-10 keV. The important parameters from the figure include maximum secondary emissive yield $\left(\delta_{\max }\right)$ with its associated energy $\left(E_{\max }\right)$. 


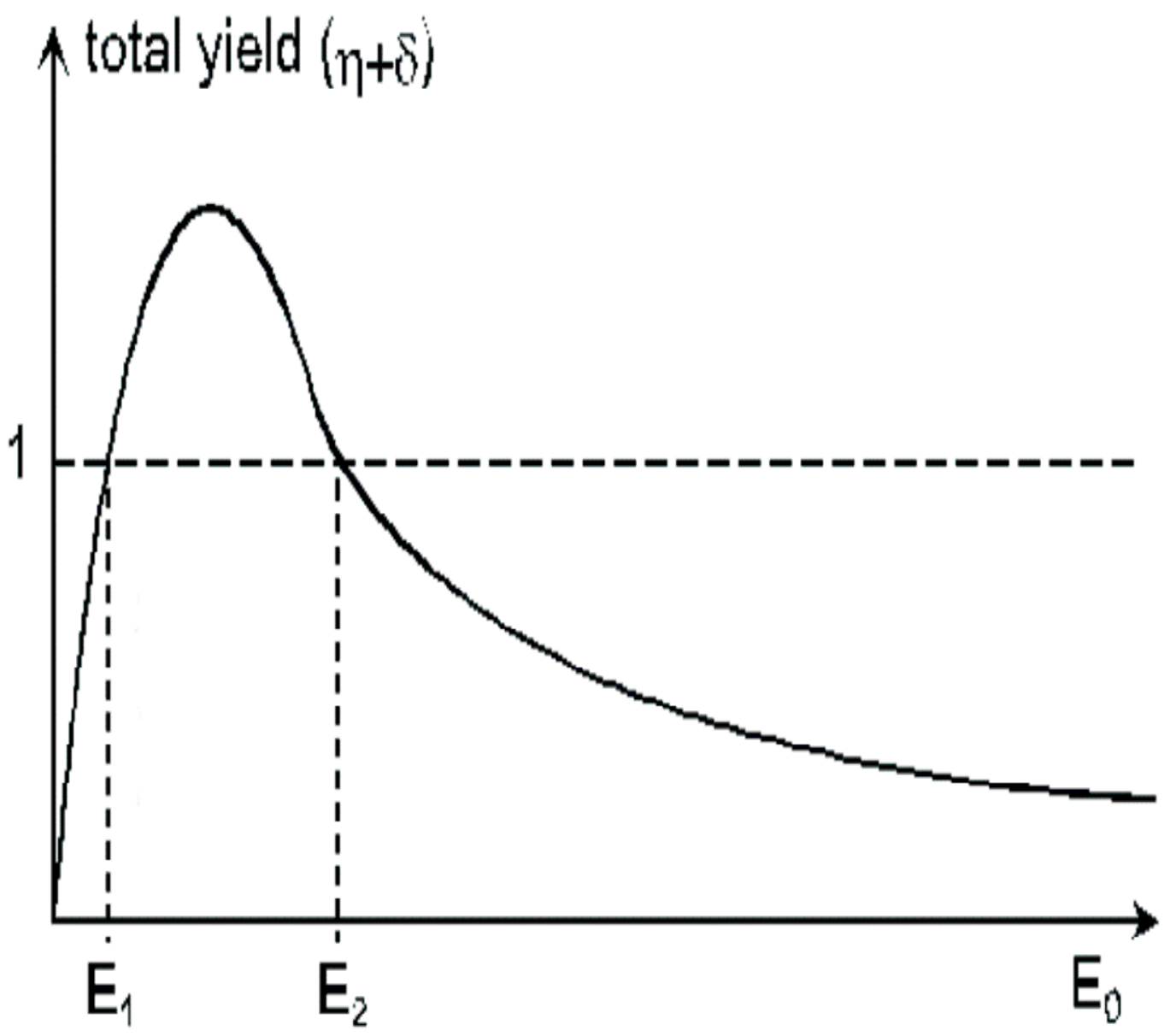

Figure 2. 8 Total yield i.e. backscattered $(\eta)$ and secondary $(\delta)$ as a function of incident beam energy $(E)$.

$$
\delta=\frac{i_{S}}{i_{p}} \text { or } 1-\frac{i_{t}}{i_{p}}
$$

Where $i_{S}, i_{p}$ and $i_{t}$ are secondary, primary and specimen current respectively, $i_{t}$ is the current which is transmitted across the sample. In this work since we have used $\mathrm{MgO}$ which is an insulator with maximum secondary yield $\sim 22$ compared to other materials [61-73], so only the theory for insulators is accentuated. 


\subsubsection{INSULATOR ELECTRON EMISSION AND SEMIEMPIRICAL MODELS}

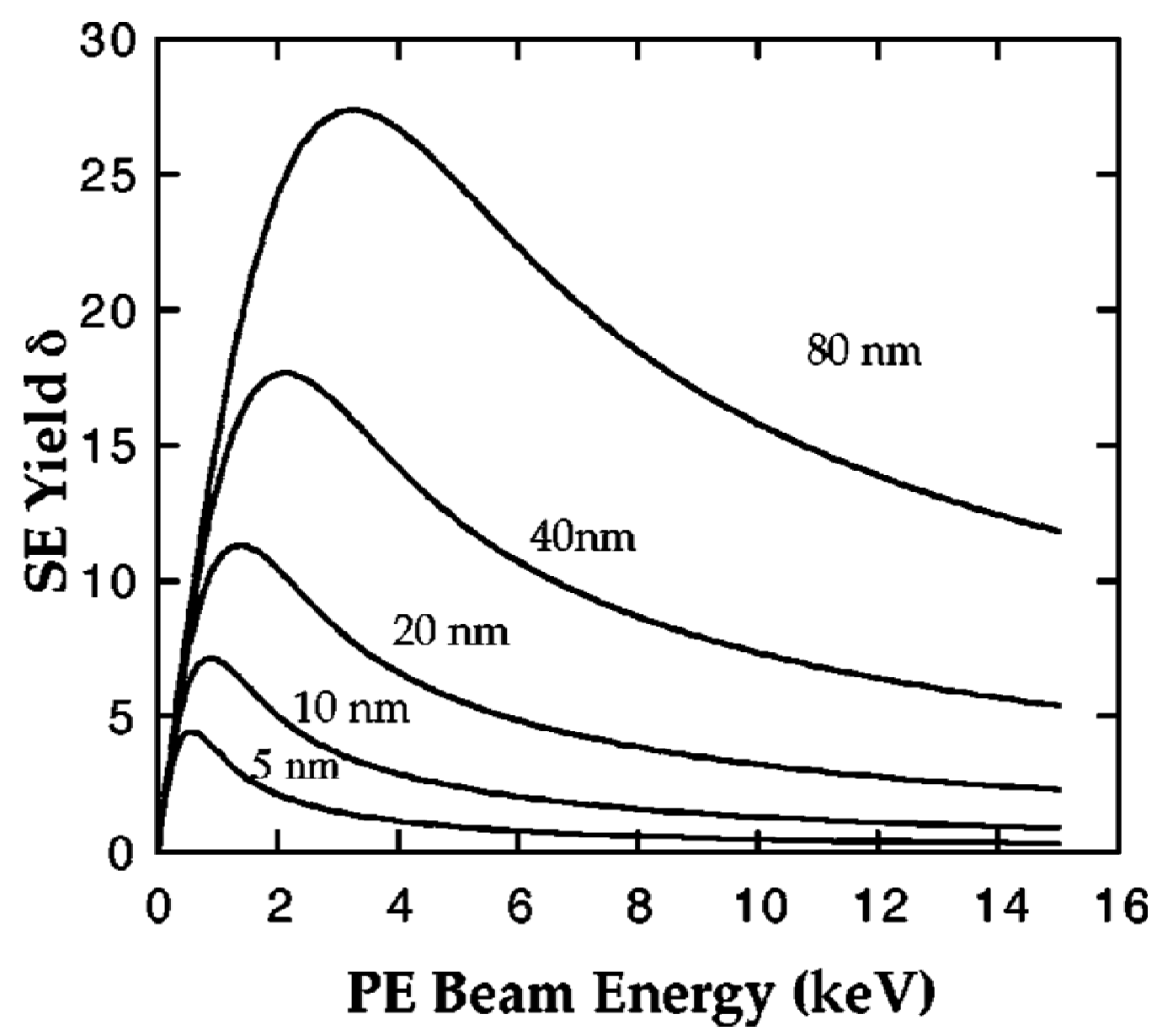

Figure 2. 9 Dependence of the SEE yield $(\delta)$ on the primary electron beam energy, for different electron escape depth [66].

This section provides a brief description about various models used in analyzing secondary electron emission particularly from insulators. An in-detail analysis and description for secondary electron emission of insulators and metals is also provided in the dissertation works of Nickles [62] and Thomson [63]. The model used by Nickles, Thomson and several other researchers explains the production, transport and escape of 
the secondary electrons; which primarily come from the inelastic electron collisions and the intrinsic lattice losses of incident electrons (bombarding the material). The penetration depth and number of secondaries produced are dependent on the incident electron beam energy and other macroscopic factors affecting the emission process are related to surface preparation and cleaning procedures. Figure 2.9 shows how $\delta$ [64] is varied as a function of incident beam energy. After secondary electron generation only a portion of them are transported toward the material surface as they undergo various energy loss mechanisms which include phonons, plasmons and other inelastic collisions. SE transport process has been modeled considering it as a diffusion process, in which the probability of an electron reaching the surface is considered to decay exponentially with depth of creation [62]. And the high escape depth of insulators is explained by various theoretical considerations as, the lack of electron-electron interaction during the transport of the generated secondaries in the insulating materials from valence to conduction band, combined to their very low or even negative affinity. This leads to escape depths of secondary for uncharged insulators in the $20-100 \mathrm{~nm}$ range instead of the $10 \mathrm{~nm}$ range for metals. Also the low electron affinity $(\sim 0.9 \mathrm{eV}$ for $\mathrm{MgO})$ in insulators permits the generated secondary electrons to escape easily into vacuum [65-67]. In addition they also need to overcome the surface potential induced by charging due to incident electron beam in-order to escape into vacuum.

Various semi-empirical models have been proposed by several researchers to explain the influence of material properties on the SEE yield curve. These theoretical considerations were based on constant loss model by Dionne [61], Seiler [67], Salow [68] Dekker [69] and Kanaya [71] which explain the production from SE from universal yield 
curve (figure 2.8). Energy require to generate a secondary electron $(\varepsilon)$ and the probability $(B)$ for it to reach the surface is given by Alig et al. [70]

$$
\begin{array}{r}
\varepsilon=2.8 E_{g} \\
B=\frac{2.5 \delta_{m} \varepsilon}{E_{\delta_{m}}}
\end{array}
$$

Where $\delta_{m}$ is the maximum SEE yield, $E_{\delta_{m}}$ is the beam energy at $\delta_{m}$, and $E_{g}$ is the band gap in $\mathrm{eV}$. According to Dionne et al. [61] SEE yield $(\delta), \delta_{m}$, penetration depth $(d)$, primary beam energy at $\delta_{m}$ and first crossover energy are given by the following equations

$$
\begin{gathered}
\delta=\left(\frac{B}{\varepsilon}\right)\left(\frac{A n}{\alpha}\right)^{1 / n}(\alpha d)^{1 /(n-1)}\left(1-e^{-\alpha d}\right) \\
\delta_{m}=0.9\left(\frac{B}{\varepsilon}\right)\left(\frac{A}{\alpha}\right)^{0.74} \\
d=C E_{p}{ }^{n}=\frac{90}{\rho^{0.8}} E_{p}^{n} \\
E_{\delta_{m}}=2.3\left(\frac{A}{\alpha}\right)^{0.74} \\
E_{1}=\frac{0.39 E_{\delta_{m}}}{\delta_{m}} \\
E_{2}=2.36\left[\left(\frac{B}{\varepsilon}\right)\left(\frac{A}{\alpha}\right)\right]^{2.86}
\end{gathered}
$$

Where $A$ is the primary electron absorption coefficient, $\alpha$ is the SE absorption coefficient or inverse mean free path, $\rho$ is the mass density, $E_{p}$ is the energy of primary electron 
beam, $n$ is the power law exponent assumed as 1.35 [61] or 1.67 [58]. $\alpha$ and $A$ values for various insulators have been provided by Khairi et al. [72]. Since the various parameters used in above equations are generally not know a more general equation has been suggested by Joy et al. [58]

$$
\frac{\delta}{\delta_{m}}=1.28\left(\frac{E_{p}}{E_{\delta_{m}}}\right)^{-0.67}\left(1-\exp \left(-1.614\left(\frac{E_{p}}{E_{\delta_{m}}}\right)^{1.67}\right)\right)
$$

It is known that the SEE yield of a polycrystalline insulator decreases due to the reduced mean free path of the SEs through scattering and trapping by defects. The effect of crystallite size on SEE yield has been modeled by Cazaux et al. according to which higher yield is observed in materials with larger grain size. This might be due to lesser energy losses or scattering events of secondary electrons such as inelastic interactions with atomic vibrations and elastic interaction with acoustic phonons or crystal defects [73]. SEE is also dependent on the angle of incidence of primary beam which can be understood as follows. If the primary electrons are falling normally on the surface the escape depth is $d$ and when they are falling at an angle it becomes $d \cos \theta$. Several researchers have proven that this is true [59, page 100]. These formulas would be used in later chapters to analyze the SEE data obtained from various materials.

\subsection{MICROCHANNEL PLATE SIMULATION}

This section provides a brief description of the effect of channel geometry and the important parameters of MCP. A MCP and the mechanism of electron multiplication are shown in figure 2.6. The gain obtained from the channels of an MCP is a function of 
various parameters which include the geometry of the channel i.e. aspect ratio and tilt, applied voltage and SEE yield of secondary emission material [11-12]. There have been several numerical models to analyze these parameters on the gain of MCP. And also there has been only one study by choi et al. [12] to determine the effect of channel tilt on the MCP gain. In this work we have utilized CPO a program based on boundary element method (BEM) which is much faster and more accurate than traditional methods used in other programs.

Figure 2.10 shows the difference in conduction of electrons in tilted and non-tilted channel. For non-tilted channels, the electric field has only an axial component. In tilted channels, there is small normal component of electric field the surface of channel wall. This weak electric field will have an affect on the electron trajectories and improve the gain of electron multipliers [12]. From the MCP simulation a practical way to achieve higher gain by changing the geometry of the channel i.e. the maximum tilt and comparison of these results with existing models and advantages of $\mathrm{CPO}$ would be presented in later chapters. 

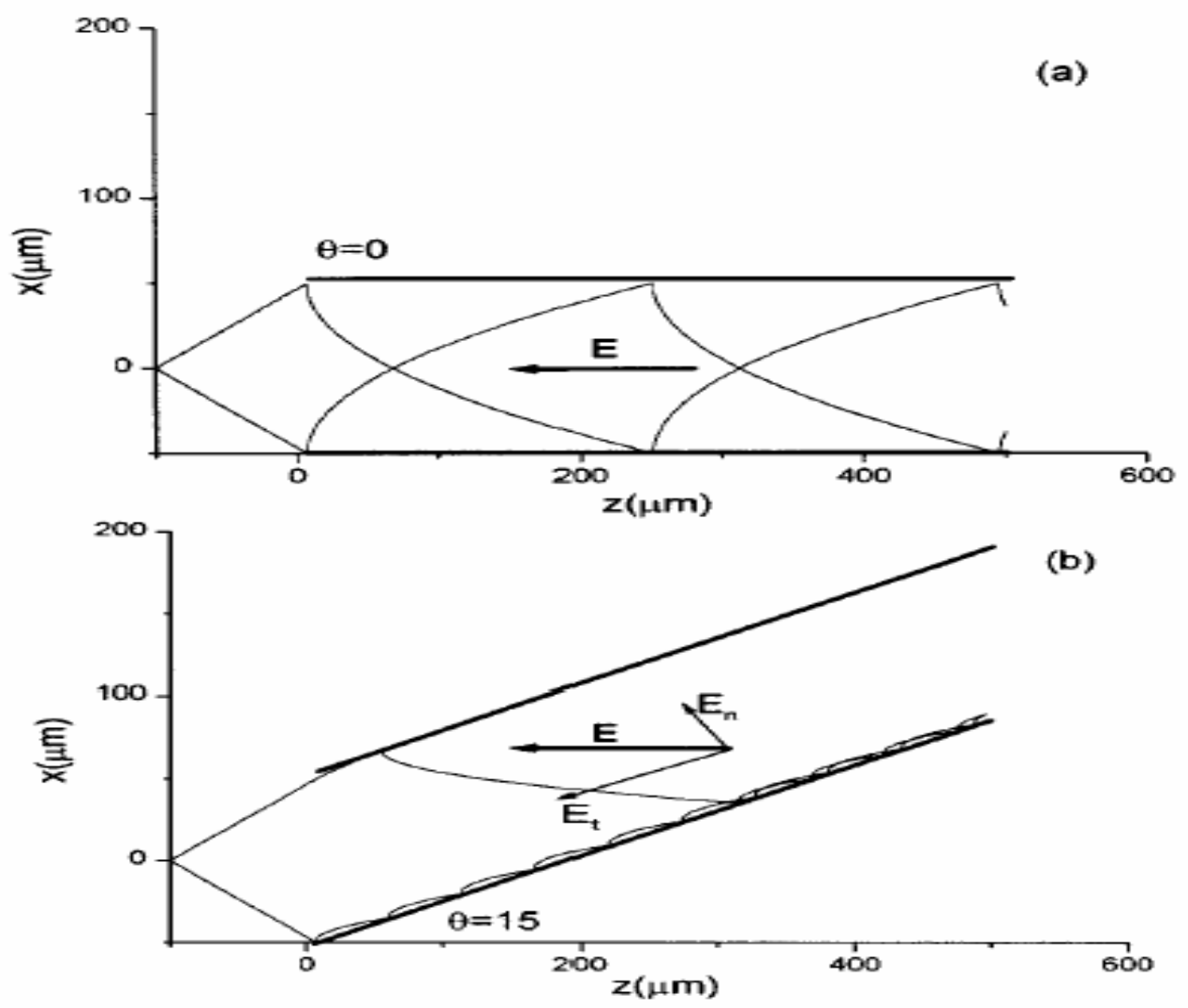

Figure 2. 10 Figure 2.10 (a) Zigzag modes in a non-tilted cylindrical channel for two primary electrons. The electric field (E) is along the channel axis, (b) a hopping mode in a tilted channel $\left(15^{\circ}\right)$ for two primary electrons. $E_{t}$ and $E_{n}$ are electric field components in the tangential and normal direction, respectively [12]. 


\section{EXPERIMENTAL PROCEDURE}

This chapter provides an overview of carbon nanotube and tungsten oxide field emitter's synthesis and the description of experimental procedure for analyzing the field emission and its enhancement with microchannel plate (MCP). This chapter also includes the description of microchannel plate (MCP) fabrication process, i.e. preparing the ceramic template, deposition methods for emissive layers i.e. $\mathrm{MgO} / \mathrm{MgO}-\mathrm{CNT}$ and resistive/metallic layers i.e. $\mathrm{CuO}, \mathrm{CNT}$ along the channel walls and surface of the MCP. Finally the experimental procedure for measuring the secondary electron emission for various materials used in this work will be described.

\subsection{CARBON NANOTUBE SYNTHESIS}

This section describes the synthesis of carbon nanotube (CNT) and tungsten oxide $\left(\mathrm{W}_{18} \mathrm{O}_{49}\right)$ multistage field emitter synthesis. In this dissertation work two types of carbon nanotube (CNT) field emitters i.e. randomly aligned thin-MWNT and MWNT array tower structures were used, which are shown in figure 3.1.

\subsection{1 thin-MWNT SYNTHESIS}

The thin-MWNTs used in this work were synthesized by thermal chemical vapor deposition (CVD) on $\mathrm{Nb} / \mathrm{SiO}_{2} / \mathrm{Si}$ substrates from a catalyst solution having Mo doped $\mathrm{Fe}$ particles supported by $\mathrm{Al}_{2} \mathrm{O}_{3}$ nanoparticles. Catalyst solution was prepared from iron (III) nitrate-nonahydrate, bis(acetylacetonato)-dioxomolybdenum(VI) and $\mathrm{Al}_{2} \mathrm{O}_{3}$ nanoparticles in methanol. Uniform suspension of catalyst in methanol with various amounts of $\mathrm{Al}_{2} \mathrm{O}_{3}$

$(30,15$, and $7.5 \mathrm{mg})$ was prepared by sonication and resulting solution was spin coated 
onto $\mathrm{Nb} / \mathrm{SiO}_{2} / \mathrm{Si}$ substrate followed by drying at room temperature and baking at $160{ }^{\circ} \mathrm{C}$ for 5 minutes in Ar atmosphere. Later the prepared substrates were placed in the CVD system (figure 3.2) followed by heating in Ar atmosphere to $700{ }^{\circ} \mathrm{C}$. At this point $\mathrm{Ar}$ was replaced by $\mathrm{C}_{2} \mathrm{H}_{4}$ gas which was maintained for 30 minutes. After completion of the above steps the CVD system was finally cooled in Ar atmosphere for 30 minutes. The role of $\mathrm{Al}_{2} \mathrm{O}_{3}$ in the catalyst solution was

(i) To support $\mathrm{Mo} / \mathrm{Fe}$ during the growth process i.e. catalyst are well distributed on the corrugated $\mathrm{Al}_{2} \mathrm{O}_{3}$

(ii) To control density, which controls the CNTs vertical alignment/orientation
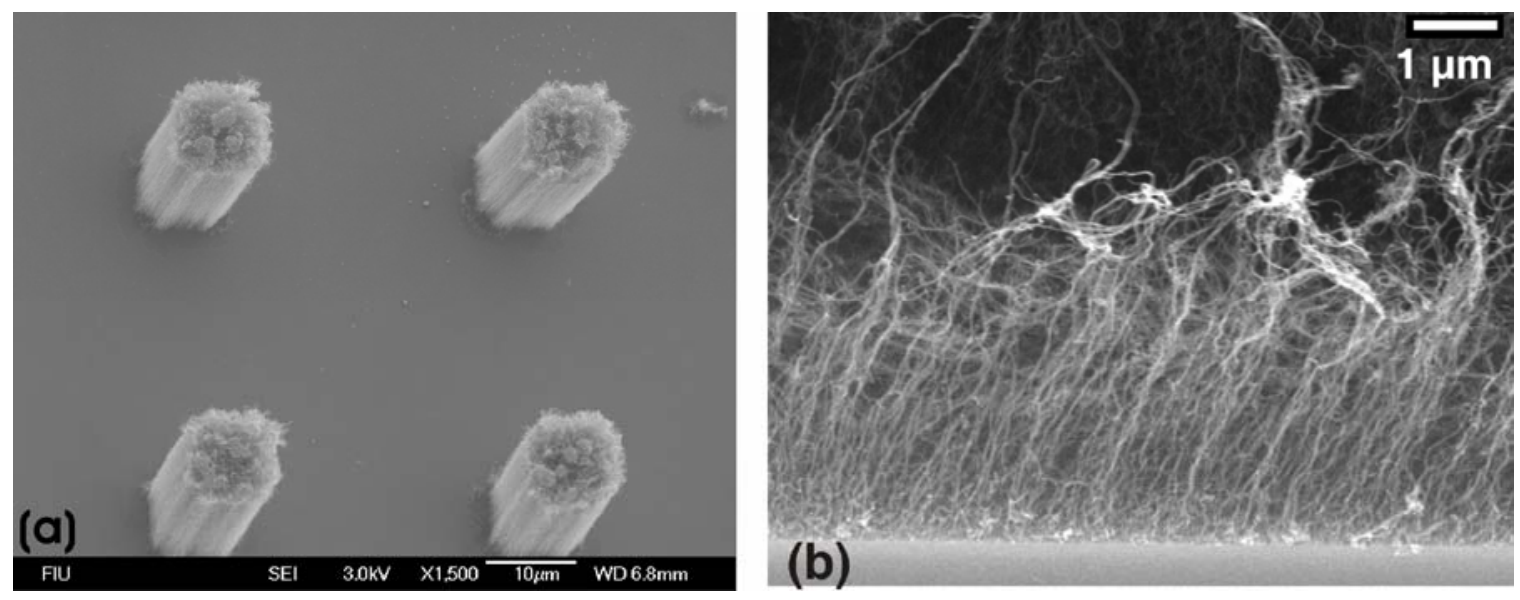

Figure 31 SEM images of (a) MWNT array tower structures synthesized on porous silicon (b) thin-MWNT synthesized on $\mathrm{Nb} / \mathrm{SiO}_{2} / \mathrm{Si}$ substrates.

Vertical alignment of nanotubes, grown by thermal CVD, is achieved by controlling their density [75]. In this work it was observed from a series of experiments that the density of thin-MWNTs (per sq-cm) was controlled by varying $\mathrm{Al}_{2} \mathrm{O}_{3}$ content in the catalyst solution. The growth of nanotubes was not uniform when grown without $\mathrm{Al}_{2} \mathrm{O}_{3}$ in 
the catalyst solution. Morphology of the synthesized nanotubes was examined by scanning electron microscopy (SEM, JEOL-7000F) and transmission electron microscopy (TEM, FEI-Technai UT). Degree of graphitization of the nanotubes was examined by Raman spectroscopy (spot size: $5 \mu \mathrm{m}$, wavelength (Ar-Ne): $785 \mathrm{~nm}$ ).

\subsubsection{MWNT ARRAY TOWER STRUCTURE SYNTHESIS}

The MWNT array tower structures used in this work were synthesized by thermal CVD on porous silicon substrates, which were prepared by electrochemical anodization/etching bulk Si wafers, in hydrofluoric acid (HF) and ethanol $\left(\mathrm{C}_{2} \mathrm{H}_{5} \mathrm{OH}\right)$ solution. Role of ethanol in the etching solution is as a facilitator for the evacuation of hydrogen bubbles developed during the process. Porous silicon can also be prepared by several other techniques which include stain/chemical etching, where no potential is applied; etching is done with $\mathrm{HF}-\mathrm{HNO}_{3}$ solution, potentiostatic i.e. voltage-controlled or galvanostatic i.e. current-controlled. Galvanostatic method is mostly preferred as etching is performed with a constant charge rate which leads to pores uniformity. Porous silicon used in this work was prepared by anodization of p-type silicon $<110>$ substrates under galvanostatic conditions in a simple O-ring Teflon cell in the dark, using an electrolyte which is a $1: 1$ mixture (by volume) of HF (48 wt\%) and ethanol (100\%) with a Pt wire as counter electrode. An aluminum plate on the backside of the sample served as an ohmic contact. The anodization was carried out at $10 \mathrm{~mA} / \mathrm{cm}^{2}$ for $5 \mathrm{~min}$ and the resulting porous silicon had a thin nanoporous layer with pore diameters in the range of $15-20 \mathrm{~nm}$. 


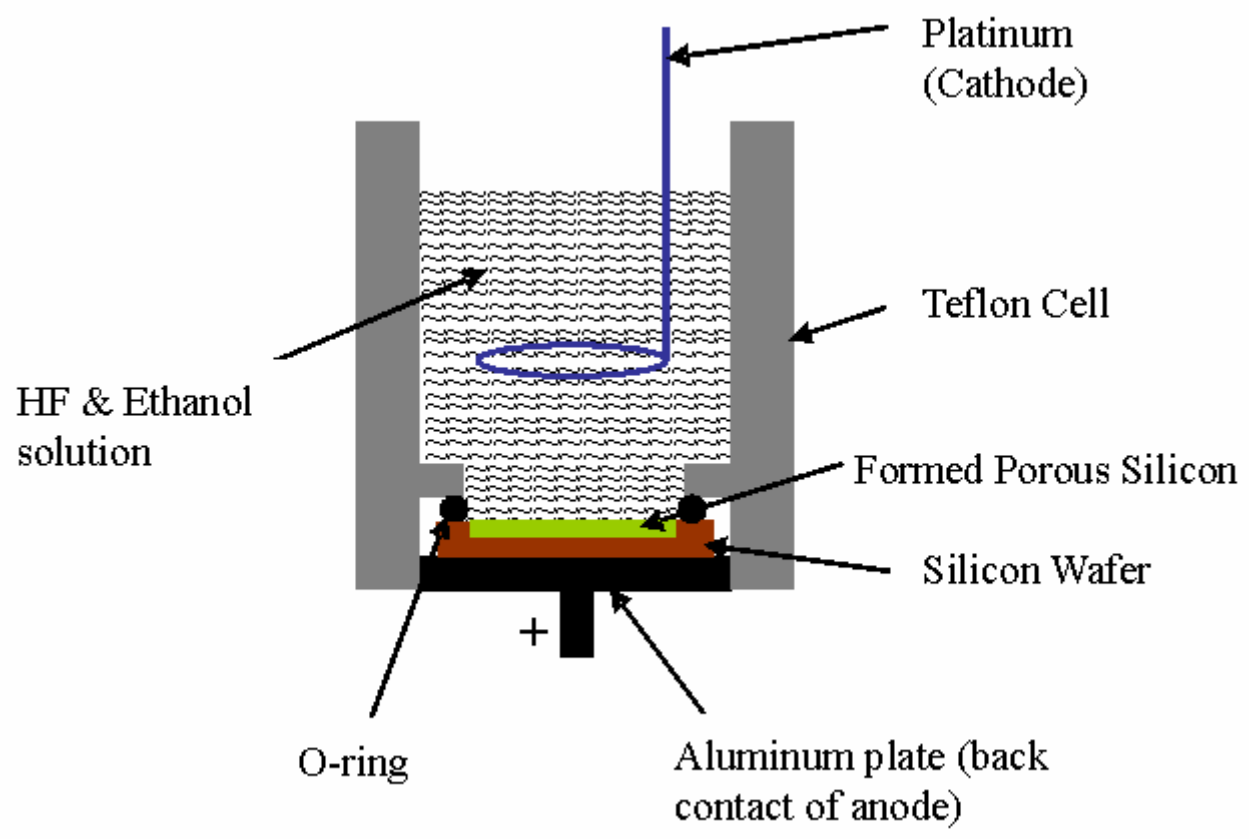

Figure 3.2 Schematic of porous silicon fabrication experimental setup.

On the porous silicon substrates a thin iron film $\sim 10 \mathrm{~nm}$ thick was sputtered through a shadow mask with circular openings. The shadow masks were prepared by laser drilling green alumina tape (un-sintered) with required pore size, which was than sintered at $1550^{\circ} \mathrm{C}$ to remove the binders. After Fe deposition the porous silicon substrates were annealed at $300^{\circ} \mathrm{C}$ for $\sim 12$ hours in a furnace under normal atmospheric conditions. Annealing at this temperature is believed to improve the contact of Fe film with pores and also reduce the stress in the porous film thus avoiding its cracking during CNT growth. Finally for the growth of MWNT array tower structures, the substrates were placed in the cylindrical quartz tube of the CVD system followed by heating in Ar $(\sim 1000 \mathrm{sccm})$ atmosphere to $700{ }^{\circ} \mathrm{C}$. At this point $\mathrm{Ar}$ was replaced by ethylene $(\sim 1000$ sccm) gas which was maintained for $40 \mathrm{~min}$, after which the furnace was cooled to room temperature for $\sim 2$ hours in Ar. Then the as grown MWNTs arrays were etched by 
oxygen plasma to remove carbonaceous content and other adsorbates on top of the CNT tips. This method also helps in achieving uniform length CNTs. For etching, the CNT substrates were placed inside the reactive ion chamber which was maintained at 120 Torr, with $10 \mathrm{sccm}$ of $\mathrm{O}_{2}$ gas, power at $100 \mathrm{~W}$, and the process was done for $3 \mathrm{~min}$. Morphology of the CNTs was examined by scanning electron microscopy (SEM, JEOL7000F) and transmission electron microscopy (TEM, FEI-Technai UT). Degree of graphitization of the nanotubes was examined by Raman spectroscopy (spot size: $5 \mu \mathrm{m}$, wavelength (Ar-Ne): $785 \mathrm{~nm})$.

\subsubsection{TUNGSTEN OXIDE NANOWIRE MULTISTAGE FIELD EMITTERS}

Multistage emitters used in this work consisted of tungsten oxide nanowires grown on electrochemically sharpened tungsten tips (figure 3.3), which were prepared from 0.006 inch diameter wire (Small Parts Inc ${ }^{\mathrm{TM}}$ ). Tip sharpening was done by electro chemical etching using $2 \mathrm{M}$ of $\mathrm{NaOH}$ solution by applying $15 \mathrm{~V}$ between the tungsten wire and the platinum electrode [76]. For the tungsten oxide nanowire synthesis, the sharpened tungsten tips were placed in the quartz tube of thermal CVD system. The synthesis process comprised of three phases which are heating phase followed by a growth phase and then cooling to room temperature. In the heating phase, tungsten tips were heated to $900{ }^{\circ} \mathrm{C}$ in $1000 \mathrm{sccm}$ of $\mathrm{Ar}$ for $10 \mathrm{~min}$. In the growth phase, the temperature was held at $900{ }^{\circ} \mathrm{C}$ for $20 \mathrm{~min}$ in a mixture of $\mathrm{CH}_{4}$ and $\mathrm{H}_{2}$, each $500 \mathrm{sccm}$. Finally the samples were cooled to room temperature in $1000 \mathrm{sccm}$ of Ar. The various parameters such as temperature, gas concentration and growth duration were systematically varied and optimized for the high yield of nanowires. High yield was 
observed at $900{ }^{\circ} \mathrm{C}$ with a mixture of $\mathrm{CH}_{4}$ and $\mathrm{H}_{2}$ : these gases have been reported to increase the yield of nanowires [77]. However, the growth duration did not have a substantial effect on the yield of nanowires. This may perhaps be due to the limited oxygen supply, which comes from the native oxide layer of tungsten tip. Morphological and micro structural characterization of the emitter was performed by scanning electron microscopy (SEM, JEOL-7000F) and Transmission electron microscopy (TEM, FEITechnai UT).
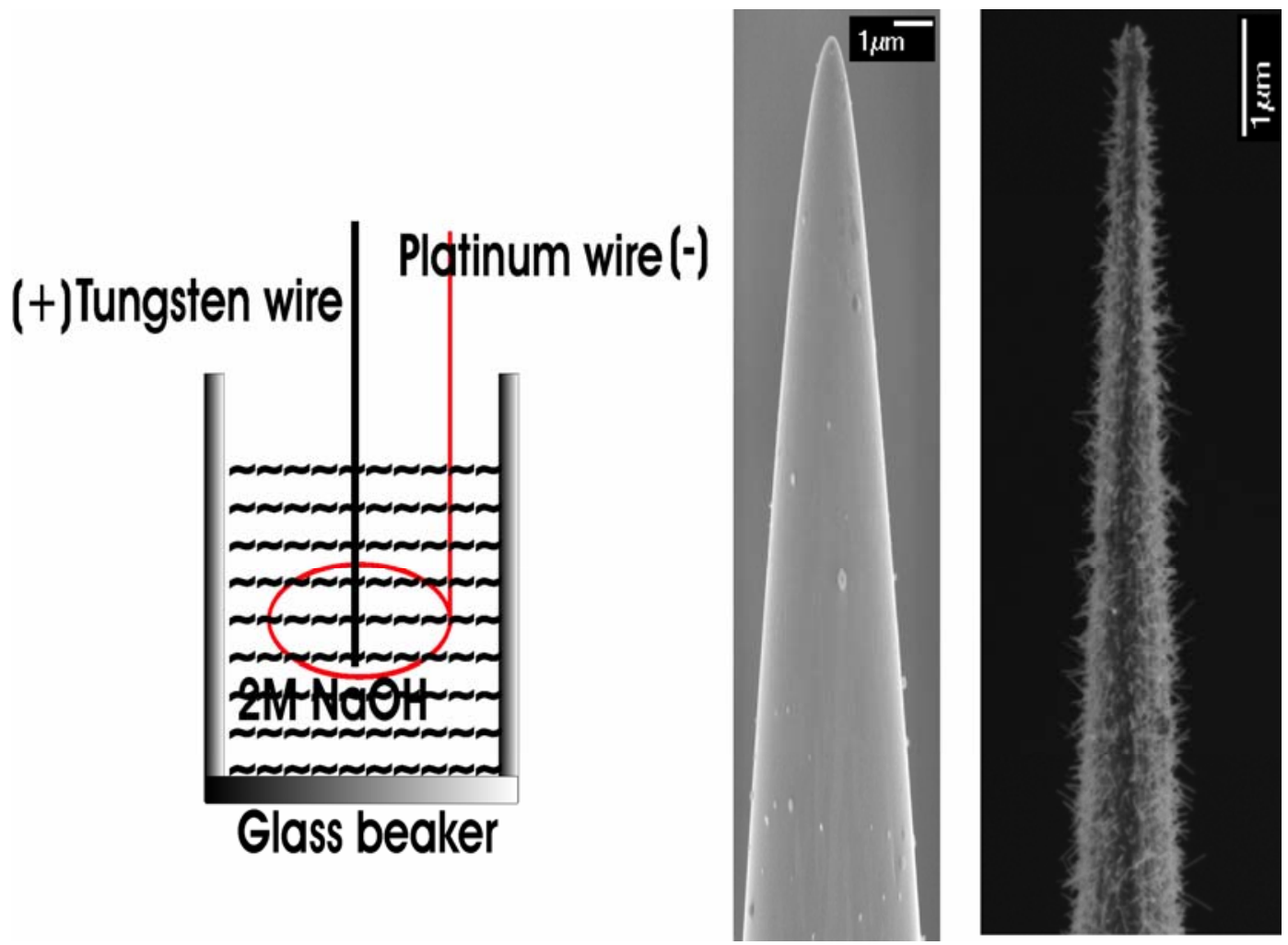

Figure 3.3 Etching setup schematic (left) SEM image as etched tungsten wire (middle) and tungsten oxide multistage i.e. nanowires on sharp tungsten tip (right). 

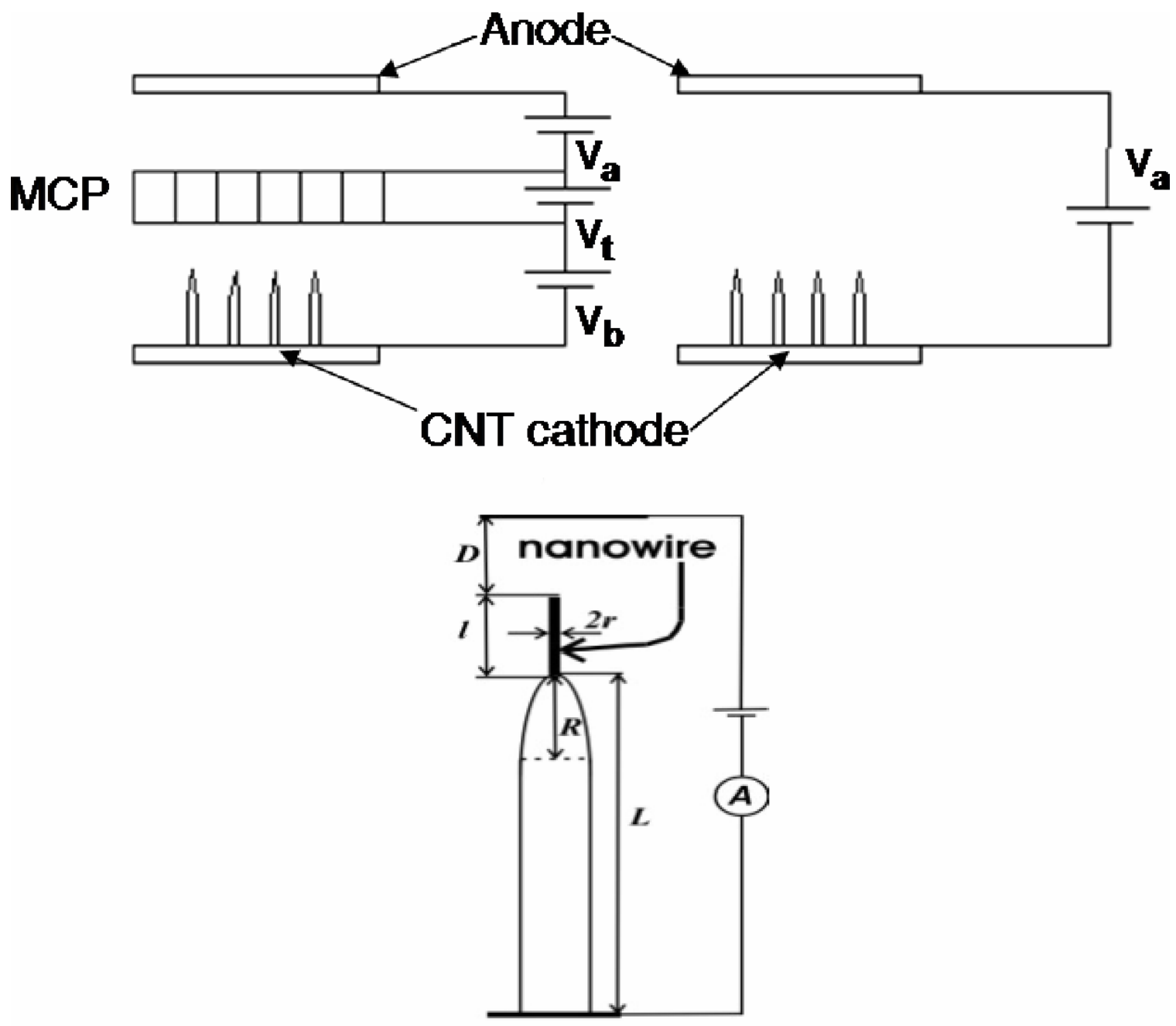

Figure 3.4 Field emission measurements circuit schematic, CNT (top) and $\mathrm{WO}_{\mathrm{x}}$ (bottom), please refer figure 2.5 in chapter for the meaning of various parameters.

Field emission measurements for CNT with and (without MCP) and tungsten oxide were performed using a diode structure with indium tin oxide (ITO) coated quartz as anode and CNTs and tungsten oxide multistage emitters as cathode in respective experiments. The cathode and anode were separated by a ceramic spacer to avoid 
electrical shorting between them. The inter-electrode i.e. cathode to anode distance (D) was in $\mathrm{WO}_{\mathrm{x}}$ and $\mathrm{CNT}$ experiments was maintained at $\sim 1150$ and $\sim 1050 \mu \mathrm{m}$ respectively.

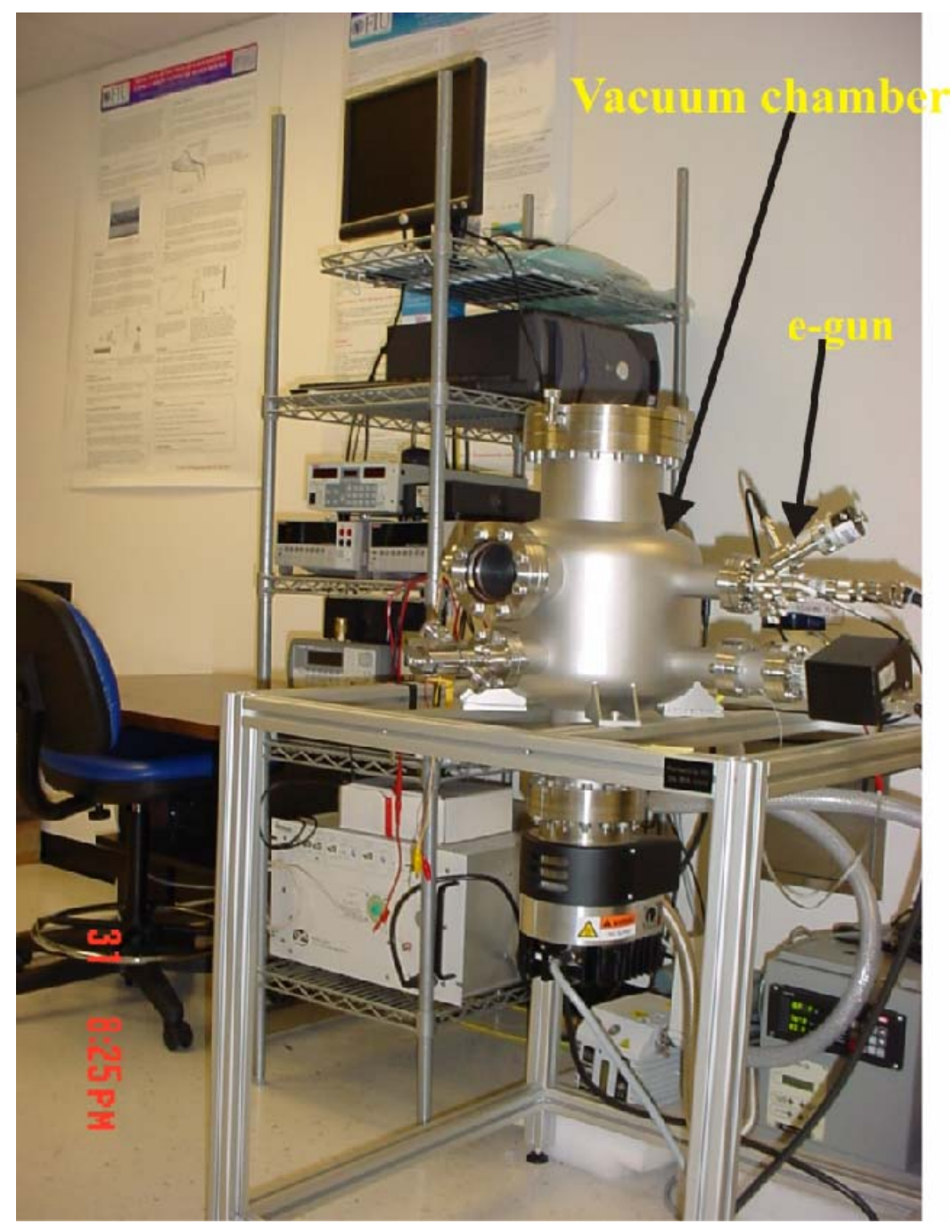

Figure 3.5 Field emission (FE) measurement system, vacuum chamber $\left(\sim 10^{7}\right.$ Torr $)$ and various measurement meters.

For studying the emission performance of $\mathrm{WO}_{\mathrm{x}}$ emitters experiments were conducted at various vacuum conditions ranging from $\sim 1 \times 10^{-6}$ to $3 \times 10^{-3}$ Torr which was achieved by a variable leak value of the vacuum chamber. Emission recovery studies were also 
performed at $\sim 1 \times 10^{-6}$ Torr after measurements at $\sim 3 \times 10^{-3}$ Torr. Schematic of measurement circuits are shown in figure 3.4. The measurement also had a protecting feature for avoiding any damage to the measurement meters from arcing and other superfluous events. The circuit protector consisted of a resistor (few $M \Omega$ ) in parallel with a Zener diode (few Farads). For current measurements Keithley 2010 multimeter (nano ampere resolution), Keithley 6487 picoammeter and for voltage source Keithley 6487 and Keithley 248 were used. All the measurements were automated through LabView. Figure 3.5 shows the measurement system and 3.6 image of LabView interface and circuit or block diagrams are shown in appendix 5. For emission enhancement experiments an MCP was inserted between the CNT cathode and anode as shown in the measurement schematic in figure 3.4. The measurements were performed in continuous and discontinuous dynode structure of MCP. In the former MCP consists of single high aspect ratio channels in which the top and bottom face of MCP is biased at different potentials. In the later several MCPs with smaller aspect ratio are stacked one over the other with a different bias applied to successive stage.

\subsection{MICROCHANNEL PLATE FABRICATION}

This section describes the fabrication procedure of the microchannel plate (MCP) used in this dissertation work. The ceramic template with channels was fabricated from alumina green tape (un-sintered) by micro drilling. A ceramic green body is a mixture of ceramic powder with appropriate binder and has hardness much lower than sintered form, which makes it easier to drill holes through it. The micron sized channels $(1-50 \mu \mathrm{m})$ were drilled by the following methods 
a) $\mathrm{CNC} /$ Laser drilling

b) Mechanical punching

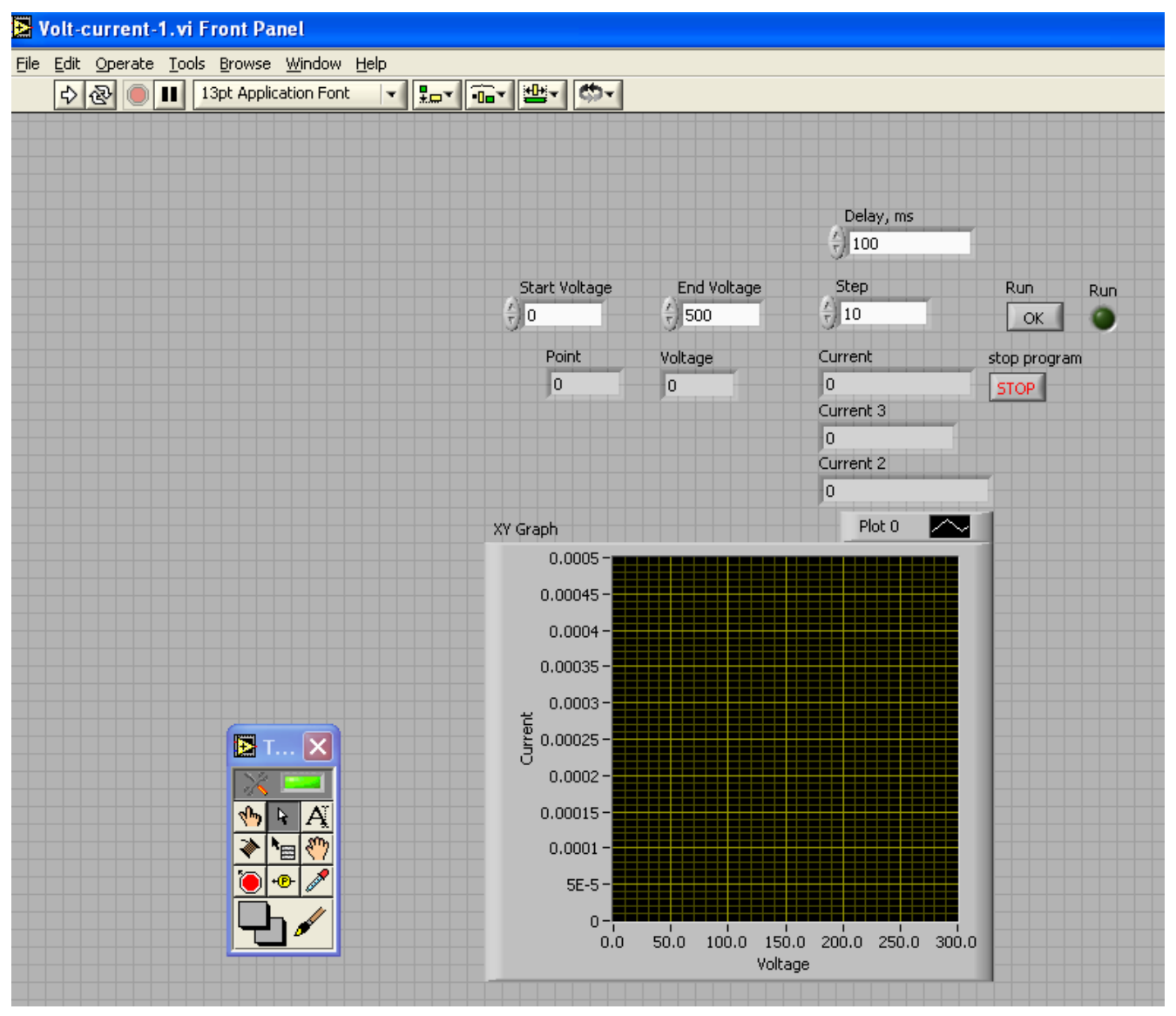

Figure 3.6 LabView interface showing the program used to measured the I-V characteristics of the field emitters. 

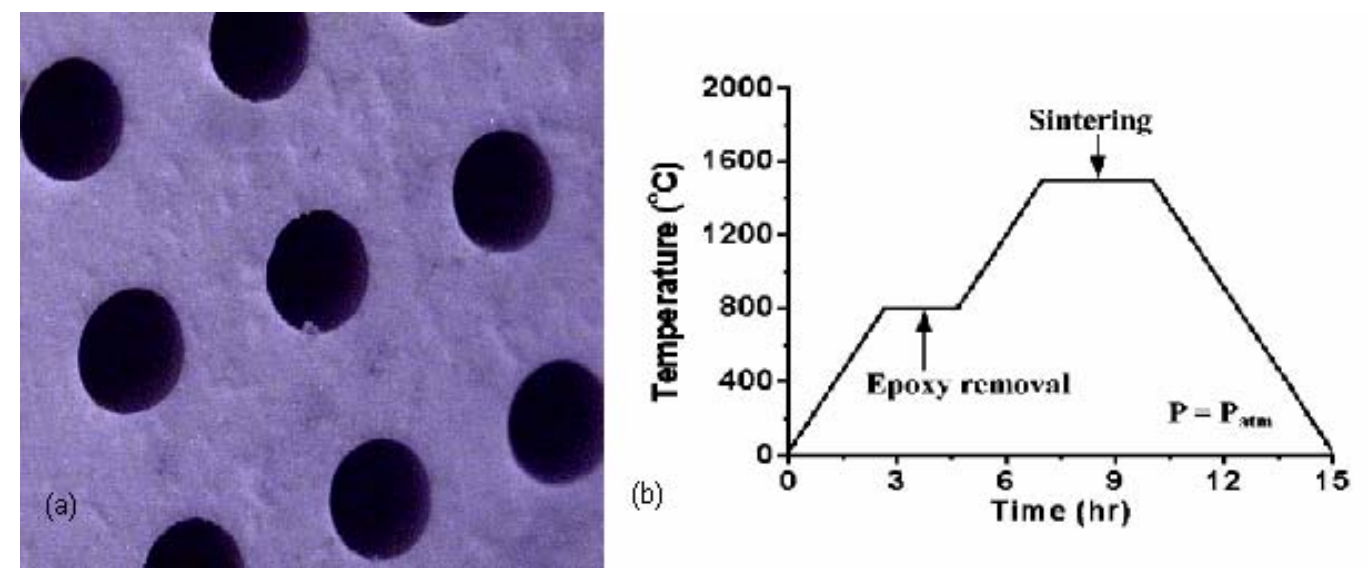

Figure 3.7 (a) Aluminum oxide/Alumina $\left(\mathrm{Al}_{2} \mathrm{O}_{3}\right)$ templates (green tape) with micron sized (b) Sintering cycle for alumina green body.

Alumina templates with micron-sized channels were stacked, laminated and sintered to obtain high aspect ratio channels. Lamination and sintering procedure is provided in appendix 1. Figure $3.7 \mathrm{a}, \mathrm{b}$ show the alumina templates and the sintering cycle, respectively. Figure 3.8 shows the cross-sectional view of an MCP and its channel. The channel walls of an MCP consist of an electron emissive and a resistive/metallic layer. Emissive layer is responsible for generating secondary electrons upon bombardment by primary electrons. Resistive/metallic layer functions as a biasing layer to which required potential can be applied and it also replenishes the electron deficiency produced in the emissive layer. In this work we had studied two materials as resistive/metallic layer, they consisted of $\mathrm{CuO}$ and multi-walled carbon nanotubes (MWNT). From our simulation and experimental work we found that MWNT is better than $\mathrm{CuO}$. For emissive layer $\mathrm{MgO}$ was as it has the highest secondary emissive yield of most materials [59-73]. Cu was coated along the channel walls of the MCP by electroless solution which was purchased from Transene chemicals. Before plating the alumina templates were etched to improve 
the adhesion of $\mathrm{Cu}$ to the ceramic surface [78-80]. The plating and etching procedure is explained in appendix 2. After $\mathrm{Cu}$ deposition the MCP was heat treated in a furnace under normal atmospheric conditions to $1000^{\circ} \mathrm{C}$ at the rate of $10^{\circ} \mathrm{C} /$ minute for the formation of copper oxide. The heating rate is also dependent on the thickness of coated $\mathrm{Cu}$ layer. Emissive layer i.e. $\mathrm{MgO}$ was deposited from sol-gel solution [81-83], sputtering and $\mathrm{MgO}$ suspension [84-87]. Most of the sol-gel solutions were prepared by utilizing diethyl amine (DEA), magnesium methoxide, magnesium acetate and 1,3 propanediol however these precursors were noticed to react with copper. So $\mathrm{MgO}$ suspensions were prepared from nano-powder with various dispersants [84-87]. For determining the secondary emission properties of these solutions they were coated on a flat substrate of $0.5 \times 0.5$ sq inch metal piece and characterization was performed as described in section 3.4. Deposition was done by electrophoretic deposition as described in references 75-79.

\subsubsection{GROWTH OF CARBON NANOTUBES ALONG THE MCP CHANNELS AND SURFACE}

The fabrication of the MCP plate is as follows, first holes of $\sim 250 \mu \mathrm{m}$ diameter were laser drilled through green i.e. un-sintered alumina $\left(\mathrm{Al}_{2} \mathrm{O}_{3}\right)$ tape, which were then aligned, stacked and laminated in a flat press. The thickness of the green template was $\sim 500 \mu \mathrm{m}$ thick. This particular thickness was chosen as it was observed to be the optimum thickness for sputter depositing iron (Fe) catalyst along the channels. The sintered $\mathrm{Al}_{2} \mathrm{O}_{3}$ template was $\sim 500 \mu \mathrm{m}$ thick with channel diameters of $240 \mu \mathrm{m}$ due to shrinkage of the green bodies. Later Fe catalyst was sputter deposited on both faces of the $\mathrm{Al}_{2} \mathrm{O}_{3}$ template. For nanotube synthesis the templates were placed in the cylindrical quartz tube of the 
chemical vapor deposition (CVD) system, followed by heating to $700{ }^{\circ} \mathrm{C}$ in $\operatorname{Ar}(\sim 1000$ sccm). After the temperature was stabilized at $700{ }^{\circ} \mathrm{C}$, Ar was replaced with $\mathrm{C}_{2} \mathrm{H}_{4}(\sim 1000$ $\mathrm{sccm}$ ) precursor gas, followed by cooling to room temperature for $\sim 2 \mathrm{hr}$. Function of CNTs grown along the channel were as metallic layer to which a potential can be applied and they also replenish the electron deficiency produced in the emissive layer ( $\mathrm{MgO})$. The field developed across the metallic layer would accelerate the secondary electrons along the channel. The CNTs on top and bottom face acted as biasing electrodes. Later $\mathrm{MgO}$ i.e. secondary emissive layer was sputter deposited on both faces of the $\mathrm{Al}_{2} \mathrm{O}_{3}$ template with CNTs. $\mathrm{MgO}$ thickness was varied by varying the deposition times and the optimum thickness for achieving high SEY was determined. Emissive layer (MgO) is responsible for generating secondary electrons upon bombardment of primary electrons from CNT cold cathode underneath the MCP (figure). Vertically aligned CNTs arrays on porous-silicon ( $\mathrm{Si}$ ) were utilized to determine the FE current enhancement from the MCP. The CNT synthesis was performed by catalytic CVD method, in brief the growth process is as follows, for CNT growth on the porous-Si Fe catalyst was sputter deposited through a shadow mask which had the similar dimensions as the sintered MCP template. It helped in aligning the $\mathrm{CNT}$ emitters with the MCP channels.

Material characterization i.e. structure and morphology of CNTs grown on $\mathrm{Al}_{2} \mathrm{O}_{3}$, MgO-CNT coating were performed by scanning electron microscopy (SEM) equipped with energy-dispersive X-ray (EDX) spectrometer, transmission electron microscopy (TEM) equipped with EDX spectrometer and a high angle annular dark-field detector (EDX-HAADF). The fabricated MCP was characterized in discontinuous dynode form for its multiplication with vertically aligned CNTs arrays synthesized on porous-Si nano- 
template. Electron multiplication was estimated by measuring the field emission current with and without MCP between the CNT cathode and anode. ITO coated glass screen printed with phosphor was used to perform the electron emission imaging in both the cases and the vacuum was maintained at $\sim 5 \mathrm{E}-7$ Torr for all the experiments. Throughout all the experiments with MCP similar distance was maintained between cathode-MCP which was equivalent to the one between anode-cathode without MCP. Figure 3.9 illustrates the MCP with MgO-coated carbon nanotubes.

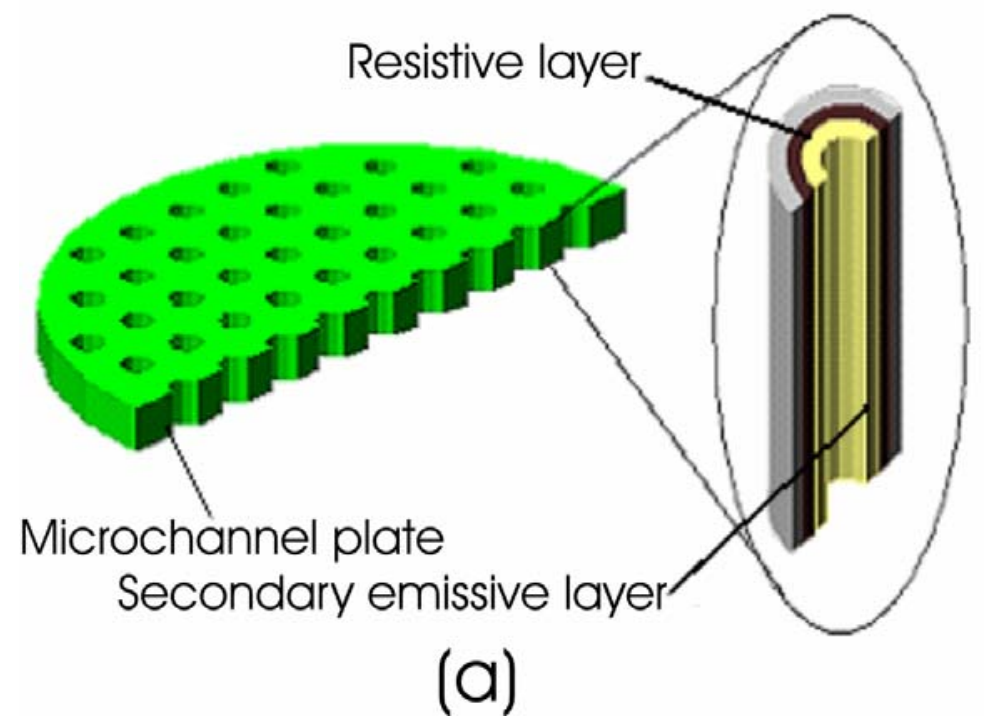

Figure 3.8 Cross-sectional view of microchannel plate and its channel. 


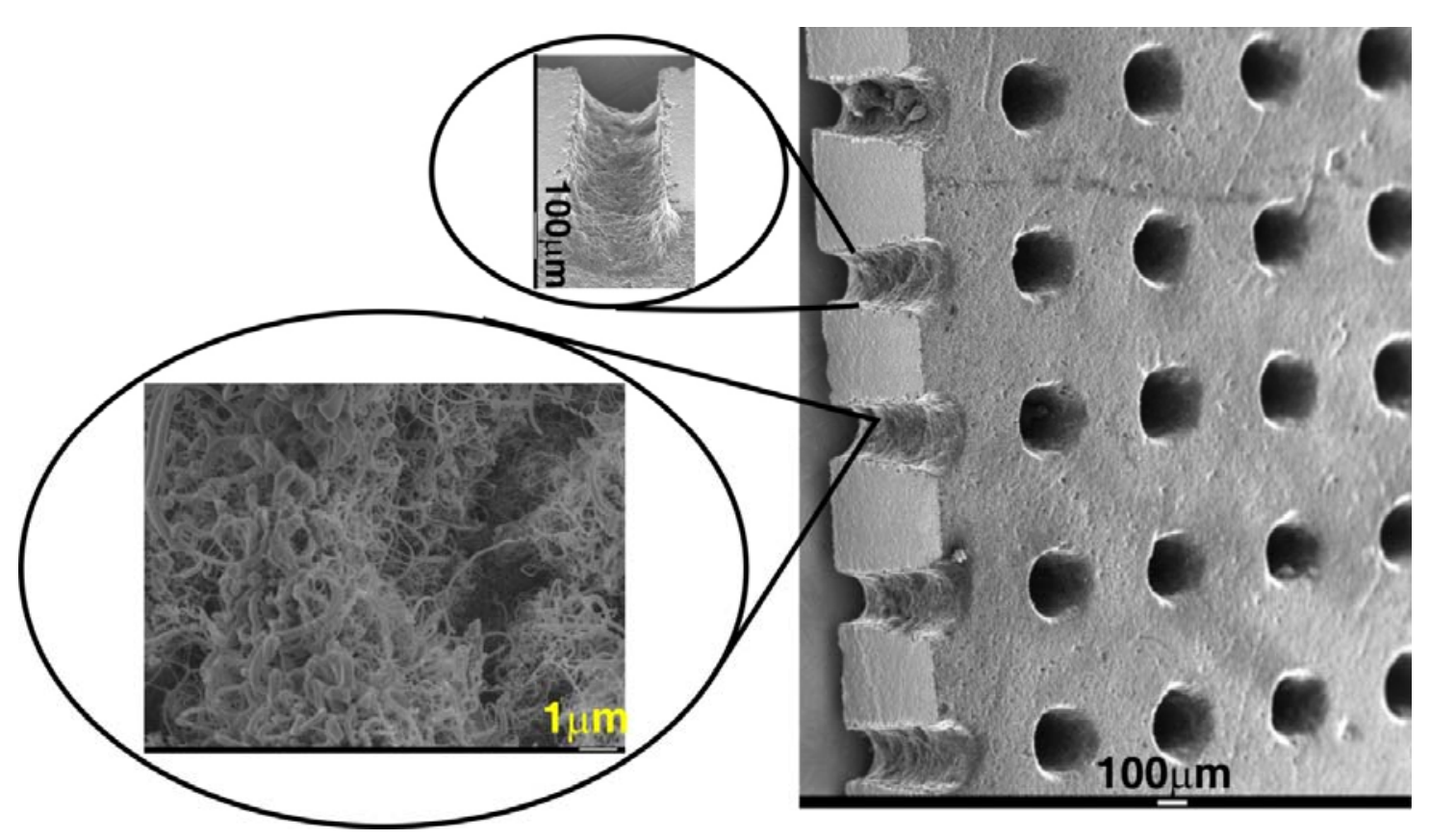

Figure 3.9 Microchannel plate consisting of MgO-coated carbon nanotubes, smaller inset shows the uniform growth of nanotubes along the channel and larger inset shows the MgO-Coated nanotubes.

\subsection{SECONDARY ELECTRON EMISSION (SEE) CHARACTERIZATION}

This section describes the secondary emission measurement for various materials. The material whose secondary emission yield $(\delta)$ is to be calculated is normally coated by various methods such as electrophoresis, sputtering etc on a flat metal surface with an area of $0.5 \times 0.5$ square inch. This plate is then inserted in a cell made out of Teflon as shown in figure 3.10. The source of primary electrons is a thermionic gun purchased from Kimball Physics (Boston, USA). Secondary electrons are generated when primaries bombard the specimen. A negative potential applied to the specimen repel the generated secondaries and $\delta$ is calculated from the following equation [1-(specimen current/input current)]. Input current is measured by a Faraday cup which is shown in the figure, for it 
the beam is focused so as to get maximum flux. The main aim of this experiment is to measure the variation of $\delta$ with incident beam energy; important parameters obtained are $\delta_{\max }$ and its corresponding beam energy.

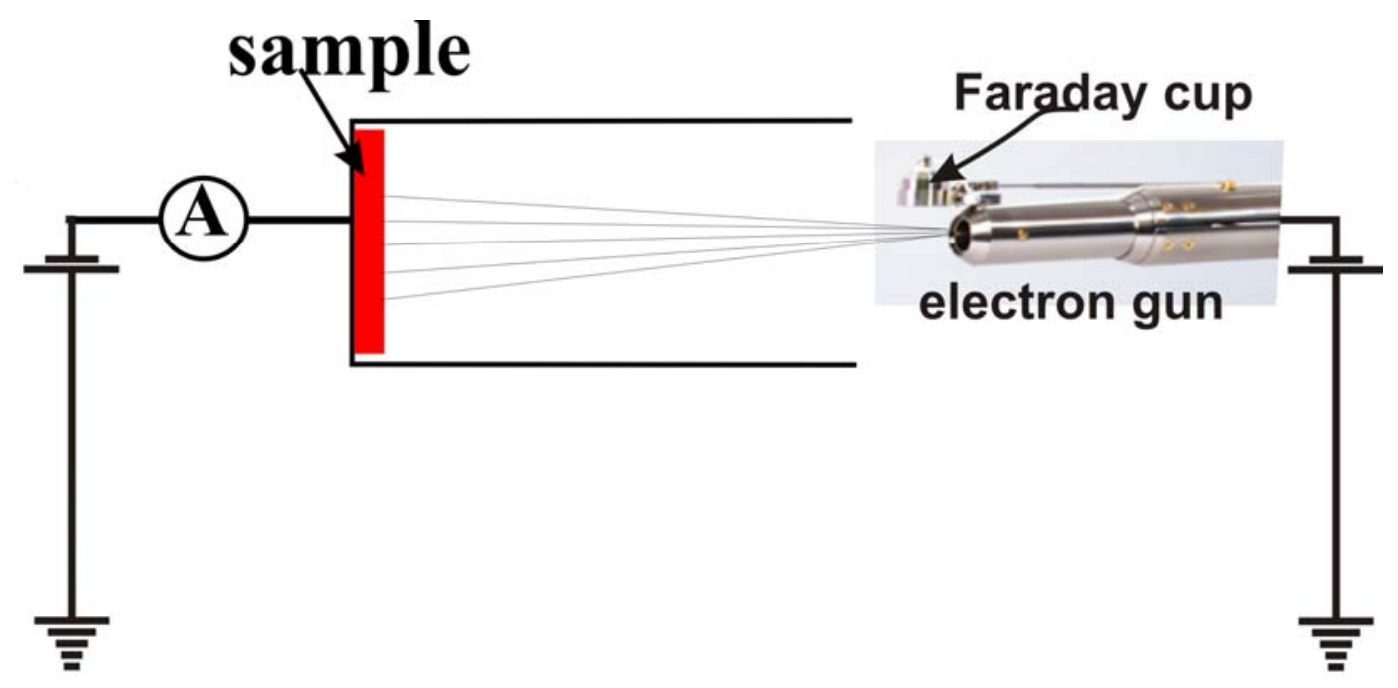

Figure 3.10 Secondary electron emission measurement cell schematic. 


\section{RESULTS AND DISCUSSION}

This chapter begins with section 4.1 , which outlines the simulation of a microchannel plate channel with various aspect ratios, tilt and material parameters. Section 4.2 outlines the characterization secondary electron emission of various materials used in this work. Section 4.3 outlines the synthesis and field emission characterization of single stage thin multiwall carbon nanotube and the multi-stage tungsten oxide and nanotube field emitters. Finally section 4.5 outlines the fabrication of the novel microchannel plate with high secondary yield materials and the amplification of field emission current from nanotube emitters through it.

\subsection{SIMULATION OF MCP CHANNEL}

Microchannel plate channel simulation was done using charged particle optics (CPO3D) which is based on boundary element method (BEM). The method is based on the fact that in a system of conducting electrodes, real charges appear on the surface of the electrodes when potentials are applied to them. In absence of leakages, these charges will remain when the leads that have carried the applied voltages are removed. These surface charges are the sources of all potentials and fields in the system. In BEM the electrodes are effectively replaced by these charges. If all the surface charges are known them all the potentials and fields are known [88]. This is ideally suited for space charge and cathode problems. A code was developed by the authors of the program according to specifications supplied by us. From the simulation two parameters for achieving maximum secondary electron yield i.e. the channel aspect ratio and the tilt were determined based on our material system. Simulation number 81,82 were utilized to 
simulate a straight and tilted channels. The procedure for setting up the simulation is provided in the appendix 3.

In the CPO3DS, 81st file one straight channel can be simulated. Channel with various dimensions i.e. diameter and length can be simulated. In the simulation the two ends of the channel are shielded with small field-free boxes. A voltage can be applied between the ends of the channel and certain number of primary electrons with a specific energy can be injected into the inlet of the channel. When an electron hits the side of the channel it produces secondaries. The energy, direction and current of the primary ray are measured when the primary hits a side and these parameters are used to determine the energy, direction and current of the secondary ray. Each ray represents a set of electrons. In principle there should usually be more than one secondary electron per primary electron, but CPO3DS cannot generate more than one secondary electron, so the currents of the rays carry the information about the number of electrons that are represented by the ray. Three options are triggered in this simulation: A Maxwellian distribution of the energies of the secondaries, Lambertian (cosine) distribution of directions, Poisson distribution of the currents. Details of these options can be found in Help section of the program. The formula for the mean multiplication factor per collision is taken from A. V. Raspereza et al [89], the formula is

$$
\mathrm{f}_{\text {mean }}=\mathrm{f}_{\max } * \exp \left(-\mathrm{a}_{\mathrm{m}} *(1 .-\mathrm{cs})\right) * 4 \cdot * x /(1 .+\mathrm{x}) * * 2
$$

Where $\mathrm{f}_{\max }$ is maximum secondary electron yield, $\mathrm{a}_{\mathrm{m}}$ is material constant,

$$
\mathrm{x}=\operatorname{sqrt}(\mathrm{cs}) * \text { energy } / \mathrm{e}_{\max }
$$

cs $=$ cosine of angle of primary to the normal to the surface, energy $=$ primary energy i.e. the collision energy of the input electrons 
According to Raspereza et al. the values of $f_{\max }, e_{\max }, a_{m}$ for most commonly used materials in MCP i.e. lead silicate are 4.0,250, 0.5 and for alumina are 4.8, 350,0.5 respectively. They also say that the secondary electrons have a Maxwellian energy distribution, mean value $2.0 \mathrm{eV}$, and a Lambertian (cosine) angular distribution. In the present example, the mean multiplication factor is used for each collision (that is, the 'mean-Poisson' option is used). This represents an approximation to the real process (see Help) but is the only viable choice here. The 'minimum incident energy' for collisions is given the value $5 \mathrm{eV}$, so if a primary ray has energy smaller than this the ray is stopped. This is the main cause of rays not reaching the anode.

\subsubsection{SIMULATION OF A STRAIGHT MCP CHANNEL}

Figure 4.1 shows the simulation results of channels with same diameter $0.01 \mathrm{~cm}$ and different aspect ratio obtained from CPO3Ds file 81 . It can be observed from figure 4.2 which is a plot of secondary emission yield $(\delta)$ vs. the channel aspect ratio that higher the aspect ratio higher the $\delta$. From the simulation results it can be noticed that higher $\delta$ value can be achieved with aspect ratio of channel greater than 40 . For the simulation secondary emissive material along the channel was assumed as alumina with an average emissive energy of $5 \mathrm{eV}$. For measuring the $\delta$ values 20 primary electrons were injected into the channel. The primary energy of the electrons was assumed as $100 \mathrm{eV}$ which is similar to field emitted electrons. Highest $\delta$ value for longer aspect ratio channels may be due to the increased number of collisions with the secondary emissive surface along the channel. Thus from the simulation it was summarized that higher the aspect higher is the $\delta$ value. 


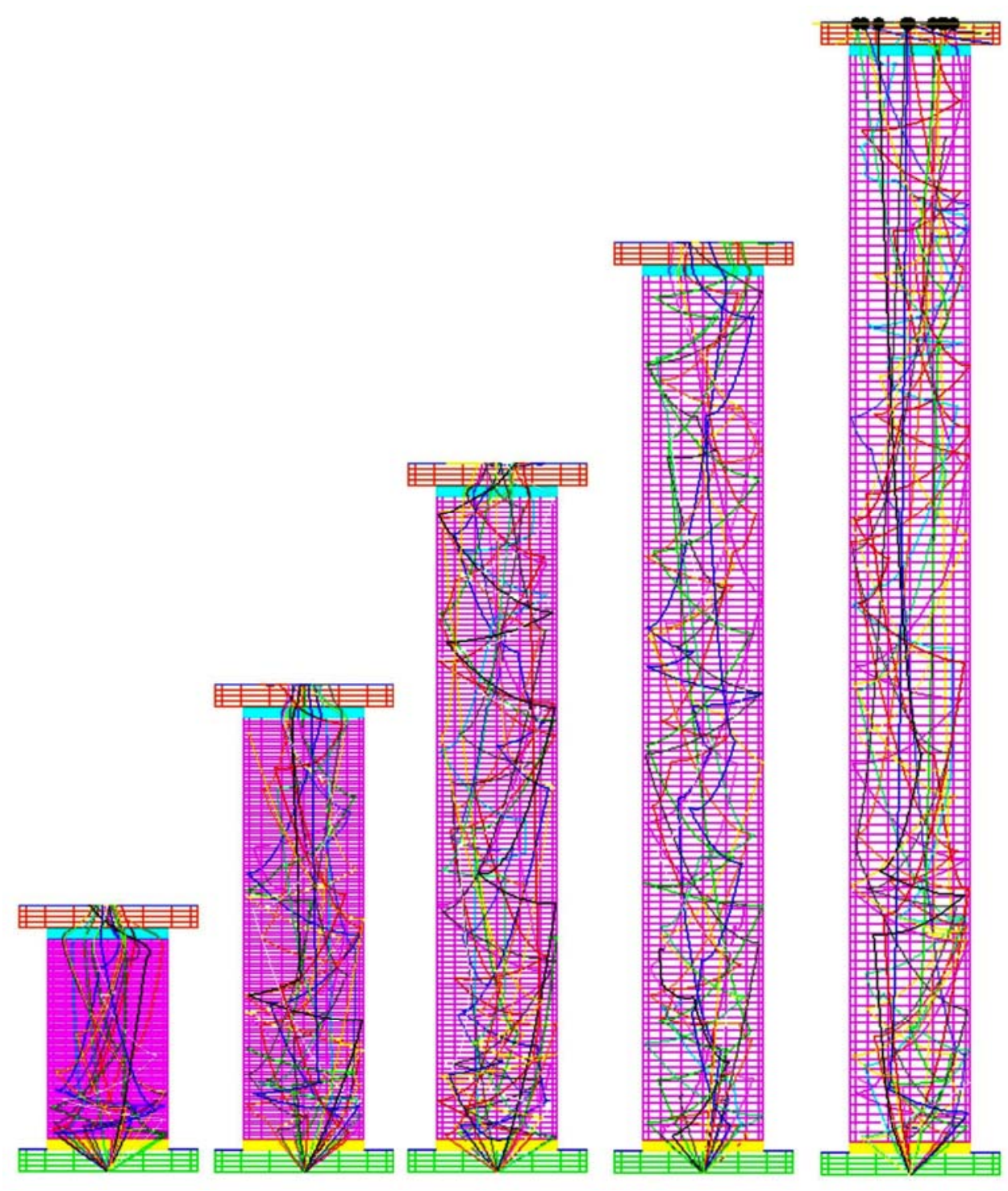

Figure 4.1 Trajectories of primary and secondary electrons for various aspect ratios MCP channels10, 20, 30, 40, 50 (left-right). 


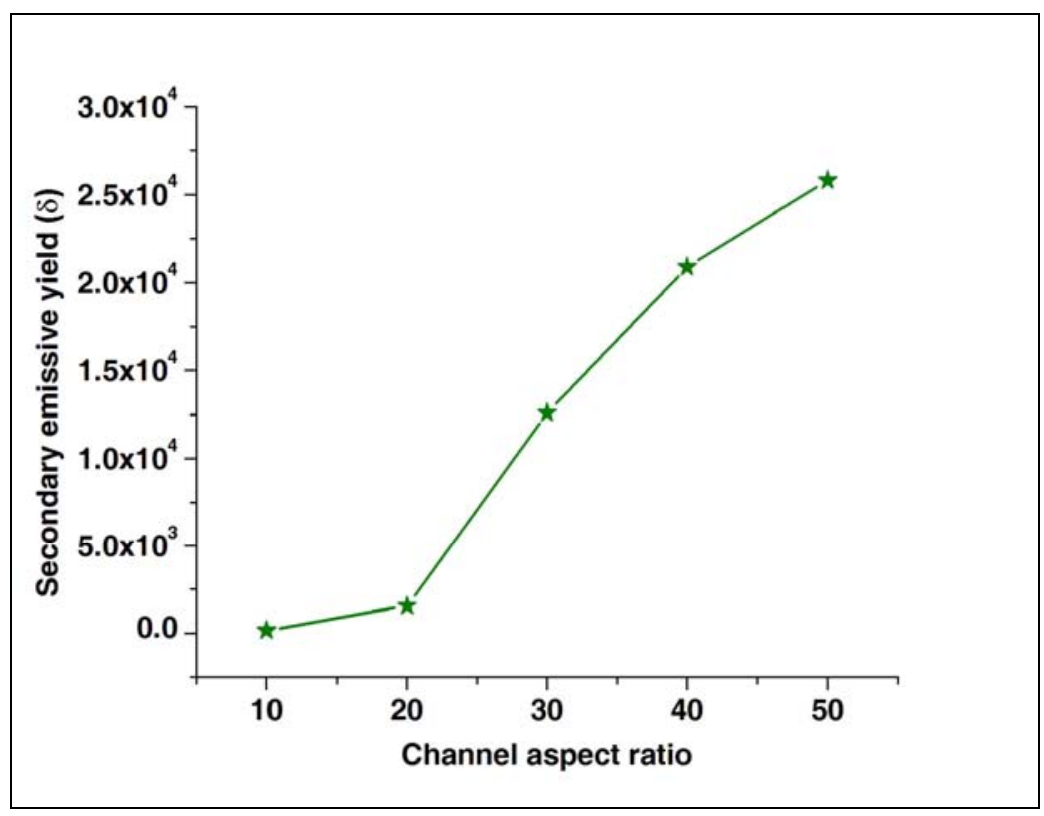

Figure 4.2 Secondary emission yields $(\delta)$ as a function of channel aspect ratio.

\subsubsection{SIMULATION OF A TILTED MCP CHANNEL}

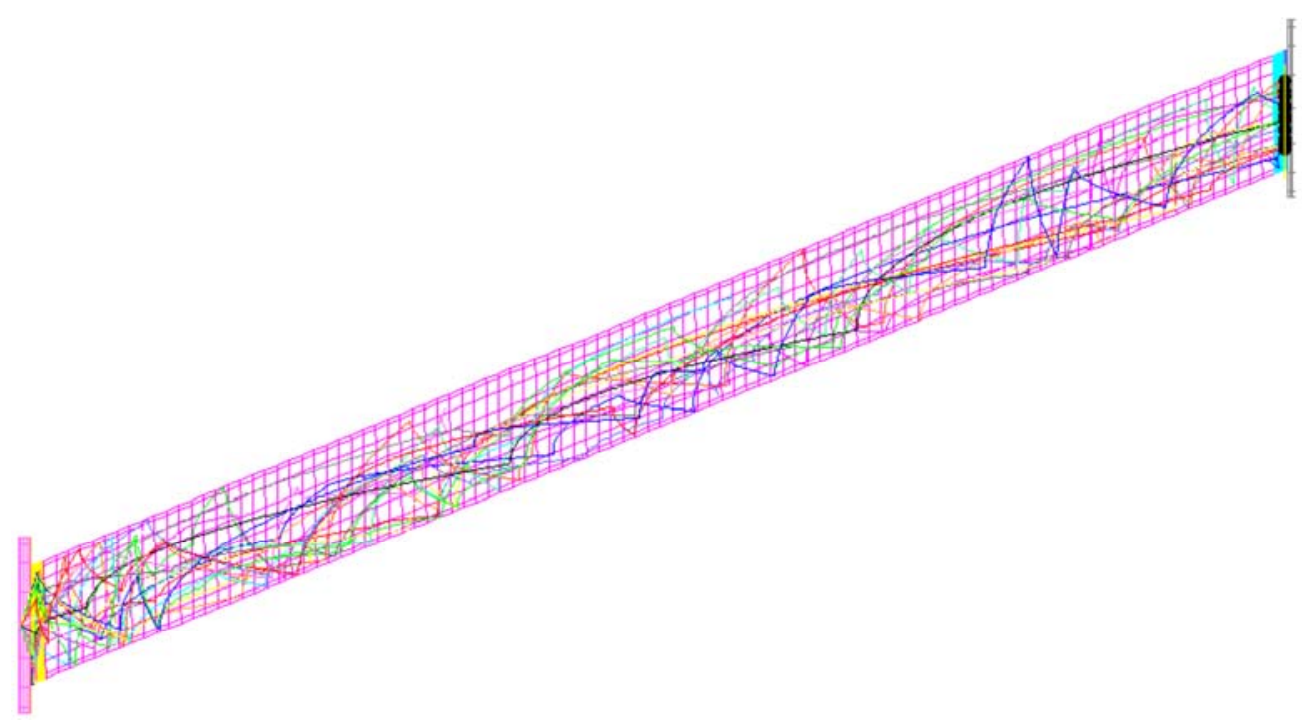

Figure 4.3 Trajectories of primary and secondary electrons for a tilted channel $\left(5^{\circ}\right)$.

The effect of the channel tilt was also simulated to determine its effect on $\delta$ value. The simulation was performed with 82 file of CPO3D, the code and procedure for which 
are given in the appendix 4 . In the simulations channel diameter was $0.1 \mathrm{~mm}$, aspect ratio was 40 and an applied voltage was $1500 \mathrm{~V}$. The secondary emissive material along the channel was assumed as alumina with an average emissive energy of $5 \mathrm{eV}$. Channel aspect ratio was chosen as 40 based on previous simulation results shown in figure 4.2 and $\delta$ value was determined by varying the channel tilt. Figure 4.3 shows the trajectories of primary and secondary electrons for tilted channels with similar dimensions of a straight channel. It can be observed that the amount of electrons and $\delta$ value is higher for tilted channels.

For the straight i.e. no-tilted channel the electric field has only the axial component, provided that the fringe field is neglected near the input and output aperture of the channel. Consequently, there is a uniform field in the axial direction. When a primary electron enters the channel with some incident angle, it strikes a point of the channel wall and induces the emission of secondary electrons. Emitted secondary electrons generally hit the opposite side of the wall. Figure 4.1 shows electron trajectories in a straight channel when secondary electrons are assumed to emit only at the normal direction to the channel wall. The trajectories are collectively on a zigzag mode. For a short channel the number of hits of the wall is small and gains are not high. This is due to the fact that the gain usually depends on the channel length and applied voltage. When a channel is tilted, the only change is the angle between the channel axis and the electric field. The change in gain is, however, outstanding. Fig. 4.3 illustrates trajectories of electrons emitted at the normal direction of a tilted channel wall. The motion of electrons is collectively on a hopping mode along one side of the channel wall. The mode may be due to an effective field on the channel wall. The electric field (E) due to applied voltage can be separated 
into the tangential component $\left(\mathrm{E}_{\mathrm{t}}\right)$ and the normal component $\left(\mathrm{E}_{\mathrm{n}}\right)$ with respect to the channel wall surface. The tangential component accelerates electrons along the channel while the normal component decelerates emitted electrons along the normal direction to the channel wall. The combination of the two fields causes electrons to have a hopping motion and strike the channel wall more frequently than the case of the straight channel. When the tilt angle is more than $5^{\circ}$ and less than $15^{\circ}$, the tilted electron multipliers are observed to produce high gains of secondary electrons. Because both the frequency of strikes and the value of collision energies are a best fit for the multiplication of electrons, the simulation results are shown in figure 4.4. Similar results were observed by Choi et al. for a cylindrical MCP channel using Monte Carlo simulation [12]. However in their results maximum $\delta$ value was observed for channels tilted at $25^{\circ}$.

From our simulation of non-tilted channels we find that many of the rays travel near the axis of the channel for a substantial fraction of the channel length between collisions, particularly after their last collision before reaching the anode. From our simulation of tilted channels we find that the rays tend to collide more frequently with the highervoltage side than with the low-voltage side and that there are no rays that travel near the axis for long distances. The highest multiplication factor occurs at approximately 15 degrees, and is then larger than that at $0^{\circ}$ by a factor of approximately 5 . At higher tilt angles the gain decreases sharply. From our simulation of tilted and non-tilted channels we conclude the optimum channel design parameters i.e. an aspect ratio of 40 and a channel tilt of $5-10^{\circ}$. 


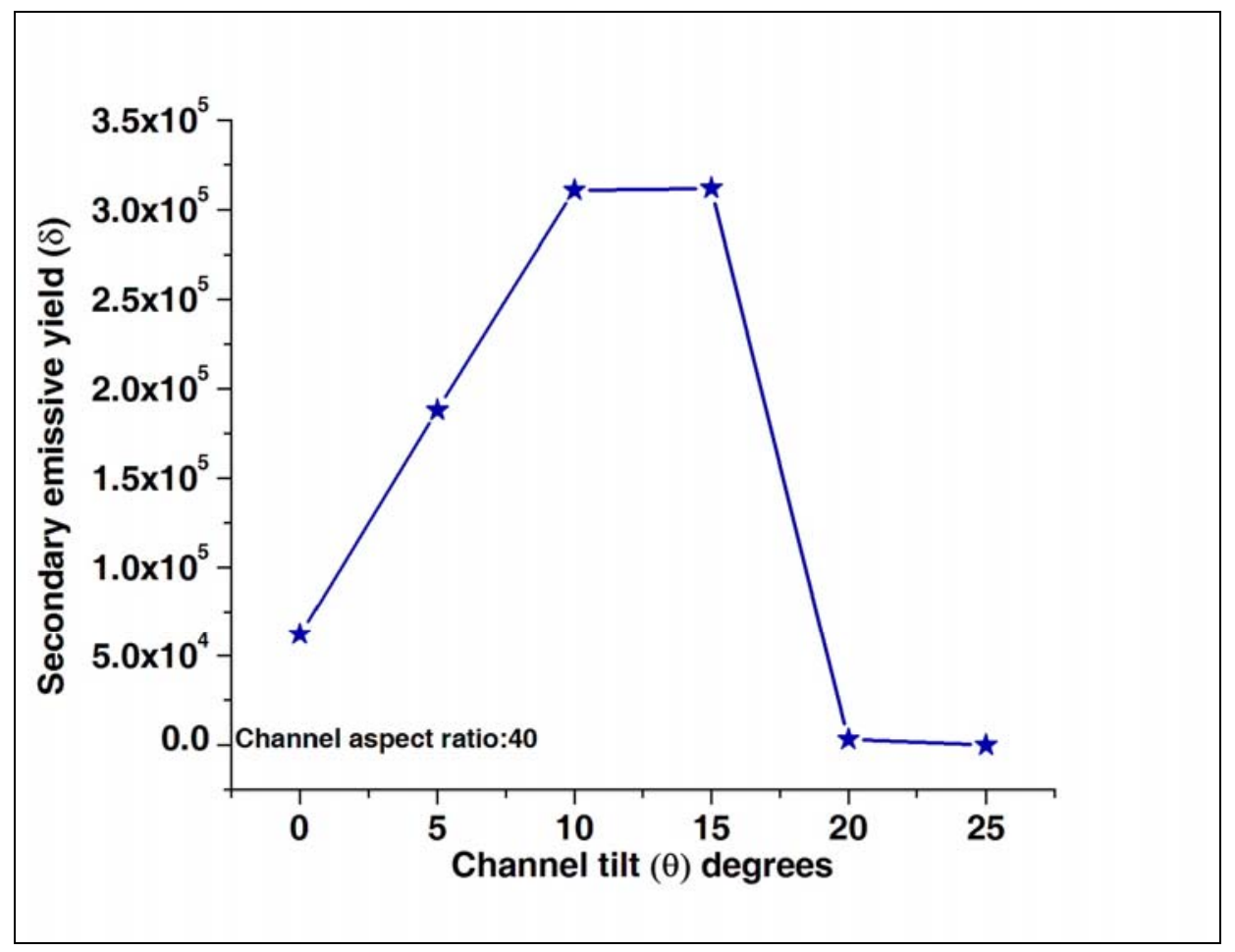

Figure 4.4 Secondary emission yields $(\delta)$ as a function of channel tilt.

\subsubsection{SIMULATION OF A MCP CHANNEL WITH VARIOUS MATERIAL SYSTEMS}

In this part of the simulation a single straight/non-tilted channel of MCP with a constant aspect ratio with various material systems was simulated. This simulation helped us in choosing the optimal material system among $\mathrm{CuO} / \mathrm{MgO}$ and $\mathrm{CNT} / \mathrm{MgO} . \mathrm{CuO} / \mathrm{MgO}$ system was chosen as electroless copper solution provided us a means to coat large aspect ratio channels. $\mathrm{MgO}$ the emissive layer was coated over the oxidized copper layer which is the resistive layer. $\mathrm{CNT} / \mathrm{MgO}$ system was chosen as it was noticed from available literature data that it had the highest secondary emission yield [13-15, 90-92]. The data required for simulating $\mathrm{MCP}$ channel with $\mathrm{MgO} / \mathrm{CuO}, \mathrm{MgO} / \mathrm{CNT}$ and lead silicate/glass 
was assumed from literature [13-15, 89-92]. Lead silicate/glass which is the most commonly used in conventional MCP was also simulated. The most optimistic values for $\mathrm{f}_{\max }, \mathrm{e}_{\max }, \mathrm{a}_{\mathrm{m}}$ for $\mathrm{MgO} / \mathrm{CuO}$ are10,500, 0.5 and for $\mathrm{MgO} / \mathrm{CNT}$ are 2500, 500, 0.5 respectively. The simulation was performed for a channel with a diameter of $\sim 250 \mu \mathrm{m}$ and aspect ratio of 2; these dimensions were chosen as these were the ones which were achievable for alumina green tape with laser facility available at the lab. Figure 4.5 shows the simulation results for various material systems from which it can be seen that $\mathrm{MgO}-$ CNT system has the highest value.

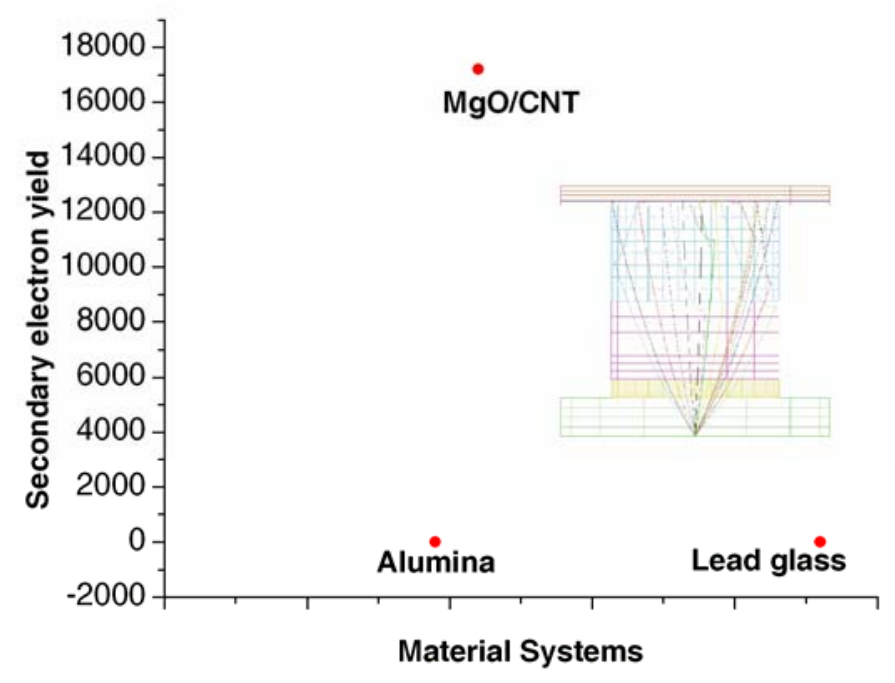

Figure 4.5 Various material system secondary emissive yields.

Even though the exact mechanism of high SEY from MgO-CNT system is not clearly understood, it is assumed as Townsend avalanche [93] that can be explained as follows. Secondary electrons are generated from the $\mathrm{MgO}$ film when primary electrons bombard it. Due to the high resistivity of the $\mathrm{MgO}$ film its surface acquires a large positive charge 
and a high field is created across the film. Subsequent electrons bombarding the film penetrate a certain distance into the film generating more secondaries. Each new secondary electron generates additional electrons by internal ionization resulting in Townsend type avalanche [93]. The higher field may also be due to the geometry of the $\mathrm{MgO}-\mathrm{CNTS}$ i.e. their aspect ratio and nano tip radius, leading to higher number of secondaries and their probability to escape into vacuum. The high SEY yields were only

observed from MCP with MgO-CNT and not with the one with only CNT. Similar kind of result was observed by Yi et. al. from their secondary electron emission and electron energy distribution studies of $\mathrm{MgO}-\mathrm{CNT}$ sample. It was concluded from their experiments that high yield can only obtained from MgO-CNT and not from CNT or $\mathrm{MgO}$ alone [13-15]. From the above simulation MgO-CNT system was chosen for our microchannel plate fabrication.

\subsection{SECONDARY ELECTRON EMISSION CHARECTERIZATION}

When electrically charged particles of sufficient kinetic energy hit the surface of a solid, the later emits electrons. These electrons are termed as secondary electrons and the bombarding electrons as primary electrons. For most of the materials, the number of secondary electrons generated by each primary electron (the secondary emission coefficient) lies between 0.2 and 3 . Generally the maximum value of secondary emission for metals is about " 1 " and an insulator is " 3 " [58-73]. The principal reason for lower secondary emission in metals is due to electron-electron scattering. In insulators there is effectively no loss mechanism other than lattice vibrations and inter-band transitions. Secondary emission in insulators can also be explained by field dependent emission. It is 
a result of conventional secondary emission, the bombarding electrons tend to charge the surface of the dielectric $(\mathrm{MgO})$ positively thus creating a high field across the film. Due to porosity, most of the primary electrons can penetrate some distance into the volume of dielectric releasing secondary electrons. These secondary electrons in turn are accelerated towards the film and at sufficiently high field, electron avalanche will occur. Due to its high secondary emission properties $\mathrm{MgO}$ was selected as the emissive layer.

The experimental setup and procedure were explained in chapter 3. Here the experimental results and its analysis are presented. Secondary electron yields are generally measured either by monitoring sample current or collecting the scattered primary and secondary emission with a retarding field analyzer or a biased Faraday cup. Each technique has defects which can contribute to potential misinterpretation of the data. We have adapted the simplest sample current method, which consists of fixing the primary electron gun potential and then determining the final current at the sample by voltage bias retardation i.e. by applying a negative potential to the sample. $\delta$ is determined from the sample $\left(I_{s}\right)$ and primary $\left(I_{p}\right)$ current as

$$
\delta=1-\left(I_{s} / I_{p}\right)
$$

Disadvantages of the method include no collection of elastically-reflected beam and no normal incidence measurement and advantages are simple equipment and stable primary beam current. 

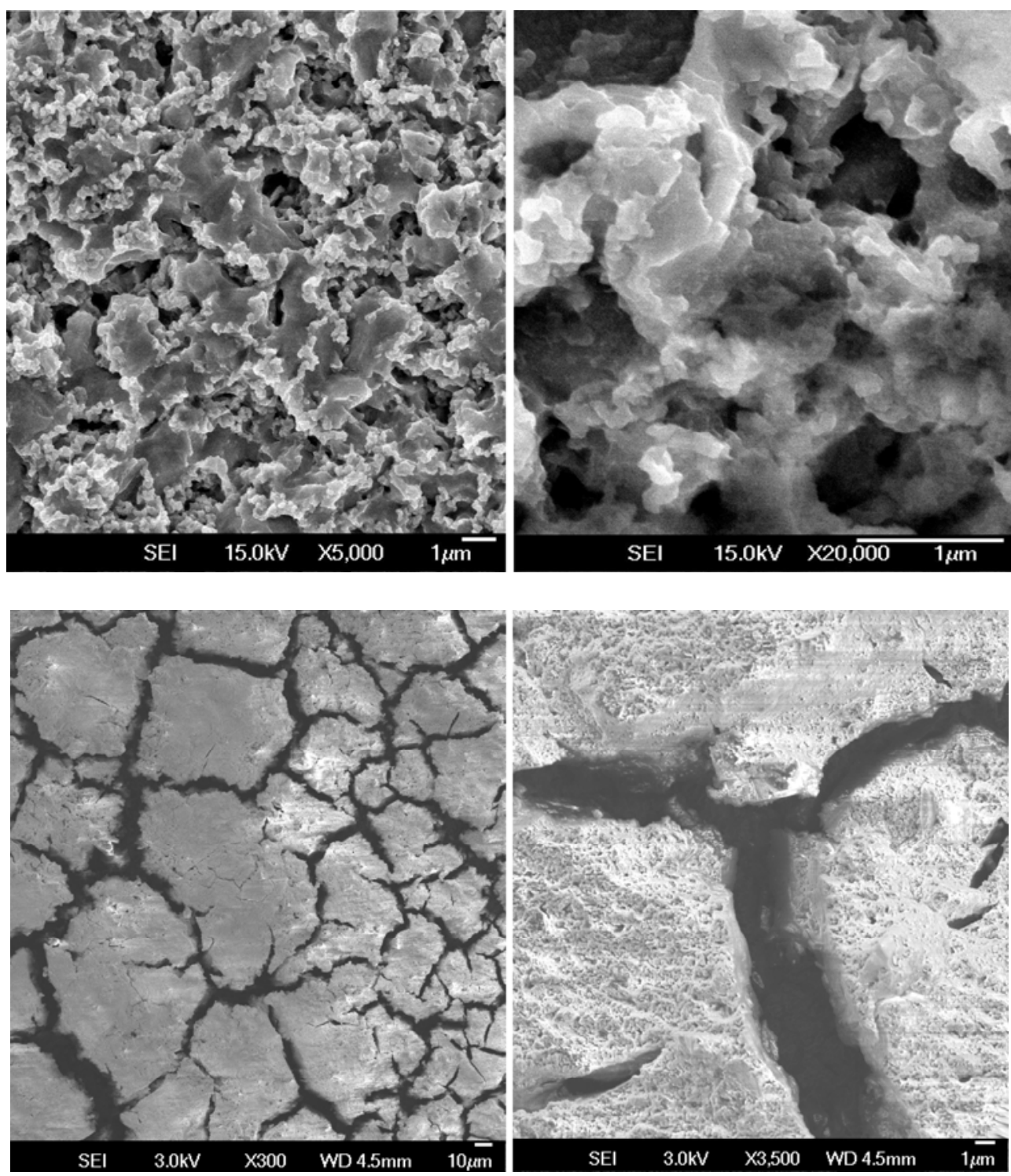

Figure 4.6 SEM images of elecrophoretically deposited $\mathrm{MgO}$ from sol-gel solution (top) and dispersant on a flat metal surface.

The secondary emission properties of $\mathrm{MgO}$ prepared by various methods and deposited on a flat metal surface were analyzed using the above method. Figure 4.6 shows the SEM image of MgO prepared from a sol-gel solution [81-83]. The difference in the surface properties of $\mathrm{MgO}$ prepared from sol-gel and suspension can be observed 
in figure 4.6. $\mathrm{MgO}$ deposited from later method seems to have larger crystal size and more porosity which might be the reason for higher SEY as shown in figure 4.7. $\mathrm{MgO}$ dispersant was composed of nano $\mathrm{MgO}$ powder with an average particle size of 15-20 nm and ethyl alcohol. A solution of citric acid and triethylamine were used as dispersant. The function of citric acid in the dispersant was to prevent agglomeration of $\mathrm{MgO}$ particles in the suspension. It is achieved due to the adsorbed citrate group on $\mathrm{MgO}$ particles and by the non-adsorbed citrate anions remaining in the solution. The adsorbed citrate leads to a negative charge on the particle surface; the non-adsorbed citrate anions contribute to increasing the ionic strength of the solution. For this reason, the use of citric acid as a dispersant not only keeps apart the $\mathrm{MgO}$ particles electrostatically but also creates a sort of steric barrier that further inhibits the complete mutual approach of the individual $\mathrm{MgO}$ particles. This particular method was adopted from literature as it observed that it produced a stable suspension from various ceramic powders as decribed by Riccardis et al. [87].

Elctrophoretic deposition (EPD) was used to deposit $\mathrm{MgO}$ because it was observed to provide reliable coatings with controlled thickness and morphology. For MgO deposition two flat metal plates of equal area separated by $\sim 1.5 \mathrm{~cm}$ immersed in the suspension were used. A DC voltage varying between $40-100 \mathrm{~V}$ was applied depending on the required $\mathrm{MgO}$ thickness followed by heating to $100-300^{\circ} \mathrm{C}$ in normal atmospheric conditions to remove the organics. Coatings prepared from EPD of MgO sol-gel did not yield good results i.e. they did not adhere to the metal surface. This may be as a result of the heating process which was followed after $\mathrm{MgO}$ deposition to remove the organics present in the sol-gel solution. However depositions prepared from EPD MgO suspensions provided 
good results in terms of coatings and secondary emission properties. Also the variation in SEY for MgO prepared from both methods is shown in figure 4.7, it can be observed from the figure that dispersant prepared $\mathrm{MgO}$ has higher SEY. This may be attributed to the morphology i.e. the crystal size, porosity of the MgO. Due to porosity, most of the primary electrons can penetrate some distance into the volume of dielectric releasing more secondary electrons as compared to $\mathrm{MgO}$ deposition obtained from sol-gel which seems to be less porous $[90,93]$. SEY of $\mathrm{MgO}$ depends on various parameters such as crystal size, surface properties, porosity $[90,93]$. High SEY values were observed from single crystal $\mathrm{MgO}$ as the secondary electron loss mechanism are relatively less in them [58-73]. Similar kind of explanation can also be provided to our observed results from $\mathrm{MgO}$ prepared from suspensions. Effects of charging were not studied here as it was not the aim and it is out of scope with the available equipment at our lab.

The similar method of coating the flat metal surface with $\mathrm{MgO}$ was not adapted for coating the channels as it was observed that it did not provide uniform layer inside the channel. This was due to variation of field concentration along the channel which is not uniform. It is usually higher at channel inlet and outlet and lesser as it reaches the channel mid section thus creating non-uniform $\mathrm{MgO}$ layer along the channel surface. However our secondary emission studies from $\mathrm{MgO}$ on flat surface provided us with the proof that high SEY can be achieved from MgO. It also provided us the information on various parameters such as $\mathrm{MgO}$ morphology and film thickness for achieving high SEY. 


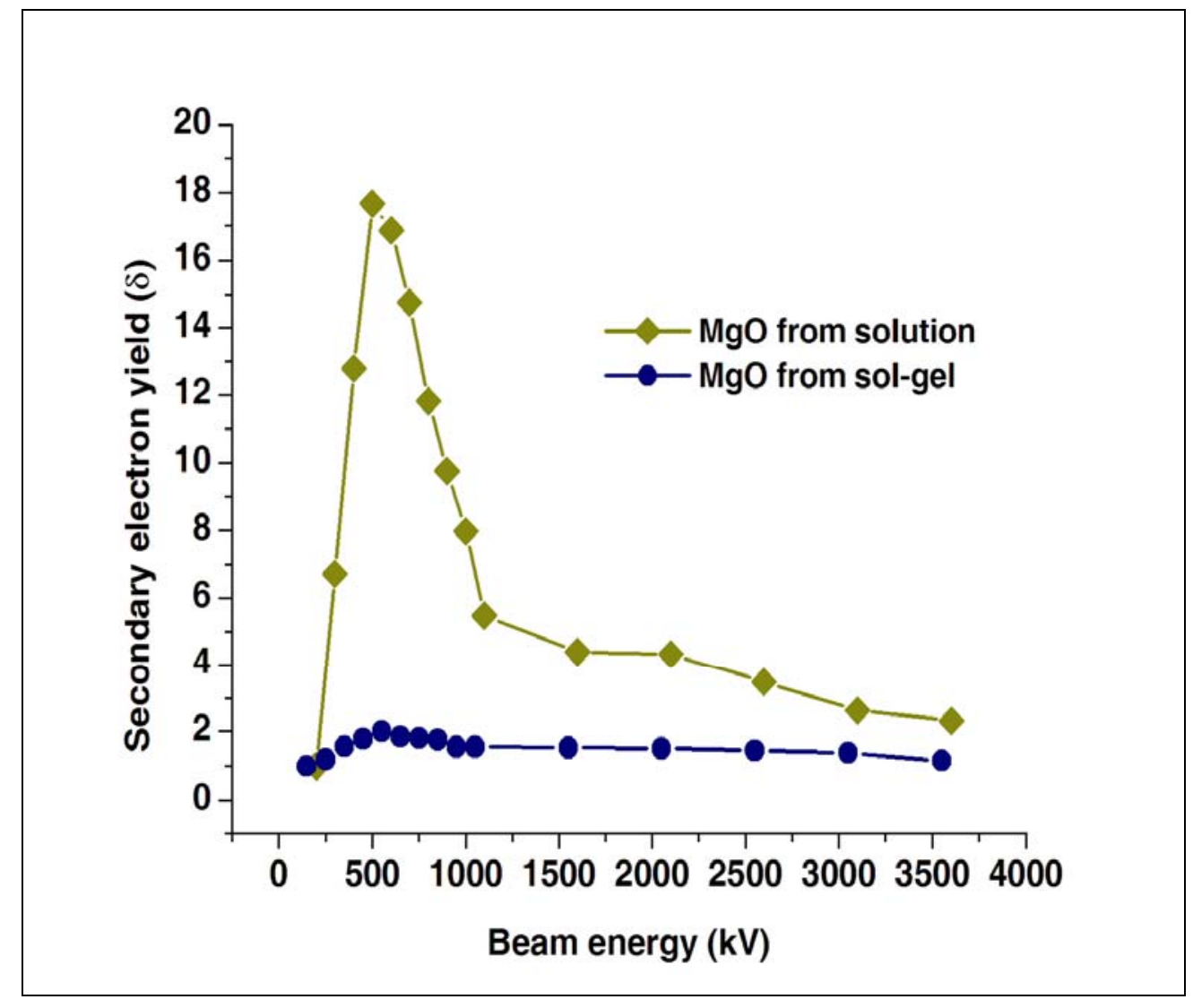

Figure 4.7 Secondary electron mission characterization of $\mathrm{MgO}$ prepared by sol-gel and suspensions.

\subsubsection{MgO-COATED CARBON NANOTUBES (CNTS) SECONDARY ELECTRON EMISSION}

From our experimental and simulation studies it was found that for achieving high $\mathrm{SEY}$ from $\mathrm{MgO} / \mathrm{CuO}$ material system, the channel aspect ratio should atleast be 40 . It is a relatively difficult process to achieve consistent coatings for such high aspect ratio channels. So a new material system i.e. MgO-coated CNTs was chosen which had shown enormously high secondary electron emission yield [13-15, 90-92] as shown in figure 4. 8. Its SEY depends on the coating thickness which is in the range of few nm to few 
microns. The minimum and maximum reported values of SEY for $\mathrm{MgO} / \mathrm{CNT}$ are 400 and 22000 , respectively [13-15, 90-92], which is the highest of all the materials. Even though the exact mechanism of high $\mathrm{SEY}$ from $\mathrm{MgO} / \mathrm{CNT}$ system is not clearly understood, it is assumed as Townsend avalanche [93] that can be explained as follows. Secondary electrons are generated from the $\mathrm{MgO}$ film when primary electrons bombard it. Due to the high resistivity of the $\mathrm{MgO}$ film its surface acquires a large positive charge and a high field is created across the film. Subsequent electrons bombarding the film penetrate a certain distance into the film generating more secondaries. Each new secondary electron generates additional electrons by internal ionization resulting in Townsend type avalanche [93]. The higher field may also be due to the geometry of the MgO-CNTs i.e. their aspect ratio and nano tip radius, leading to higher number of secondaries and their probability to escape into vacuum. The high SEY yields were only observed from MCP with MgO-CNT and not with the one with only CNT. Similar kind of result was observed by $\mathrm{Yi}$ et. al. from their secondary electron emission and electron energy distribution studies of MgO-CNT sample. It was concluded from their experiments that high yield can only obtained from $\mathrm{MgO}-\mathrm{CNT}$ and not from $\mathrm{CNT}$ or $\mathrm{MgO}$ alone [13-15, 90-92]. Figure 4.5 shows the simulation results SEY of various materials from which it can be observed that $\mathrm{MgO} / \mathrm{CNT}$ has the highest value. 


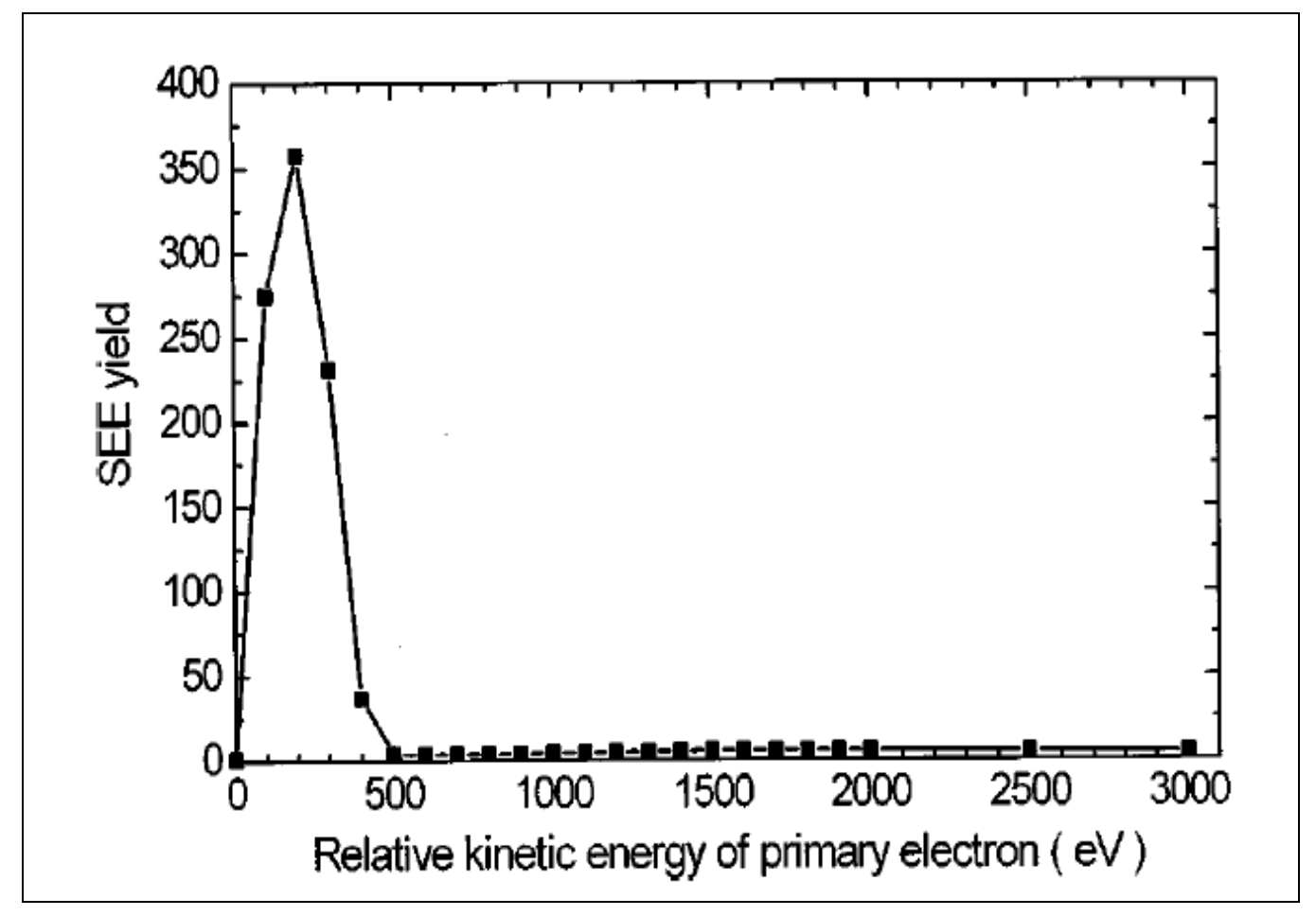

Figure 4.8 SEE yield of $\mathrm{MgO} / \mathrm{CNT}$ as a function of the relative kinetic energy of a primary electron.

\subsection{SYNTHESIS AND FIELD EMISSION CHARACTERIZATION OF thin- MWNTs}

The thin-MWNTs were synthesized by thermal chemical vapor deposition (CVD) on $\mathrm{Nb} / \mathrm{SiO}_{2} / \mathrm{Si}$ substrates from a catalyst solution having Mo doped Fe particles supported by $\mathrm{Al}_{2} \mathrm{O}_{3}$ nanoparticles. Catalyst solution was prepared from iron (III) nitrate-nonahydrate, bis(acetylacetonato)-dioxomolybdenum(VI) and $\mathrm{Al}_{2} \mathrm{O}_{3}$ nanoparticles in methanol. Uniform suspension of catalyst in methanol with various amount of $\mathrm{Al}_{2} \mathrm{O}_{3}$ (30, 15, and $7.5 \mathrm{mg}$ ) was prepared by sonication. Catalyst solution was spin coated onto $\mathrm{Nb} / \mathrm{SiO}_{2} / \mathrm{Si}$ substrate followed by drying at room temperature and baking at $160{ }^{\circ} \mathrm{C}$ for 5 minutes in Ar atmosphere. The prepared substrates were placed in the CVD system and heated to 
$700{ }^{\circ} \mathrm{C}$ in $\mathrm{Ar}$ atmosphere. Ar was replaced by $\mathrm{C}_{2} \mathrm{H}_{4}$ after the temperature was stabilized at $700{ }^{\circ} \mathrm{C}$ and gas flow was maintained for 30 minutes. The CVD system was finally cooled in Ar atmosphere for 30 minutes.

The role of $\mathrm{Al}_{2} \mathrm{O}_{3}$ in the catalyst solution was (i) to support $\mathrm{Mo} / \mathrm{Fe}$ during the growth process (catalyst are well distributed on the corrugated $\mathrm{Al}_{2} \mathrm{O}_{3}$ ) and (ii) to control density of CNTs. Vertical alignment of nanotubes, grown by thermal CVD, is achieved by controlling their density. In this work it was observed from a series of experiments that the density of thin-MWNTs (per sq-cm) was controlled by varying $\mathrm{Al}_{2} \mathrm{O}_{3}$ content in the catalyst solution. The growth of nanotubes was not uniform when grown without $\mathrm{Al}_{2} \mathrm{O}_{3}$ in the catalyst solution. Morphology of synthesized nanotubes was examined by scanning electron microscopy (SEM) and transmission electron microscopy (TEM). The average length of nanotubes from SEM measurements was approximately $15 \mu \mathrm{m}$. Figures $4.9 \mathrm{a}, \mathrm{b}$ illustrate the tilted $\left(45^{\circ}\right)$ SEM images of thin-MWNTs. TEM image in figure $4.9 \mathrm{c}$ confirms the synthesis of thin multiwall carbon nanotubes. The average outer diameter was measured to be approximately $10 \mathrm{~nm}$ with a range of 7 to $15 \mathrm{~nm}$ and the innermost wall diameters were in the range of 2.5 to $5 \mathrm{~nm}$. The synthesized nanotubes have an aspect ratio $(l / d)$ as high as 1500 (nanotube length $(l)=15 \mu \mathrm{m}$ and diameter $(d)=10 \mathrm{~nm}$ ). No catalyst particles were observed at the tube end from TEM images (figure 4.9c); so we suggest that the thin-MWNTs were grown through base growth mechanism as suggested by Fan et al. [48]. Some catalyst particles were seen inside the nanotubes indicated by marked circle in figure $4.9 \mathrm{c}$. 

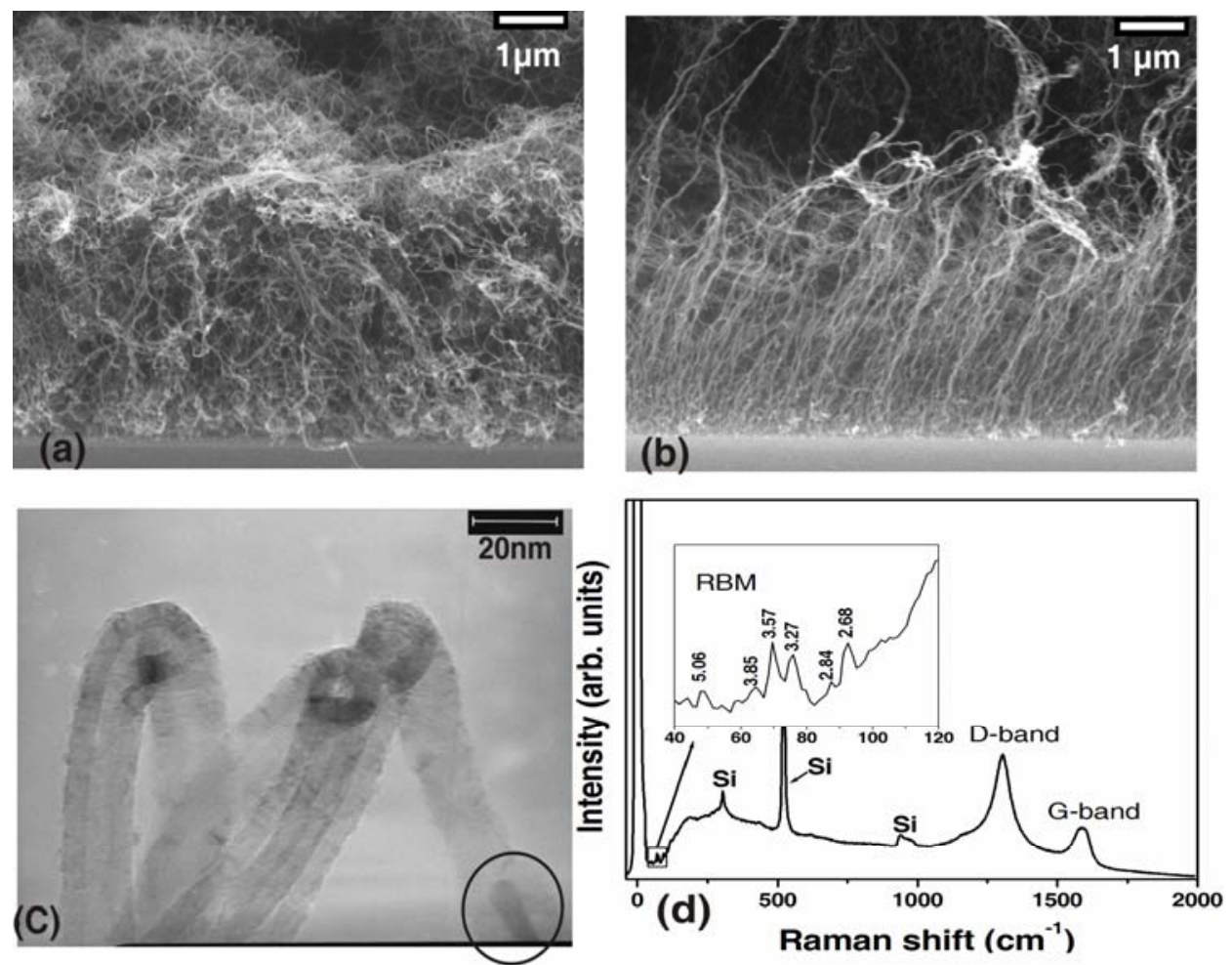

Figure 4.9 SEM images of thin-MWNTs with (a) $15 \mathrm{mg}$ and (b) $7.5 \mathrm{mg}$ of $\mathrm{Al}_{2} \mathrm{O}_{3}$

nanoparticles in catalyst solution (c) TEM image of thin-MWNTs (d) Raman spectrum of thin-MWNTs (laser excitation wavelength of $785 \mathrm{~nm}$ ) with Ar-Ne laser and inset radial breathing mode (RBM) peaks, numbers correspond to inner tube diameters.

Degree of graphitization of the nanotubes was examined by Raman spectroscopy (spot size: $5 \mu \mathrm{m}$, wavelength (Ar-Ne): $785 \mathrm{~nm}$ ). Tangential G-band located at around $1590 \mathrm{~cm}^{-1}$ (figure 4.9d) indicates the formation of graphene sheets. Relatively large Dband peak was observed at around $1300 \mathrm{~cm}^{-1}$ which is attributed to the carbonaceous particles, defects in curved graphene sheet and tube ends, and also due to finite size of crystalline domains of nanotubes [94-95]. Although we have observed very few carbonaceous particles from SEM and TEM analysis, a large intensity D-band peak was detected in Raman spectra. This may be caused by low growth temperature, which is 
similar to MWNTs grown by microwave plasma enhanced CVD at same temperature [95]. The inset of figure $2 \mathrm{~d}$ shows the radial breathing mode (RBM) peaks from $40-100$ $\mathrm{cm}^{-1}$ which is generally not observed in MWNTs. This may be attributed to the thinness (2.5-5 nm) of synthesized nanotubes. Inset of figure $4.9 \mathrm{~d}$ denotes diameters of nanotubes obtained from the relation

$$
d_{t}=248 / \omega_{R B M}
$$

Where $d_{t}(\mathrm{~nm})=$ nanotube diameter, $\omega_{R B M}\left(\mathrm{~cm}^{-1}\right)=$ wavelength of RBM peaks. Peaks located below $40 \mathrm{~cm}^{-1}$ were not detected because of strong Rayleigh scattering. The diameters determined from RBM peaks ranged from 2.68 to $5.06 \mathrm{~nm}$, which is in good agreement with innermost wall diameters obtained by TEM measurements.

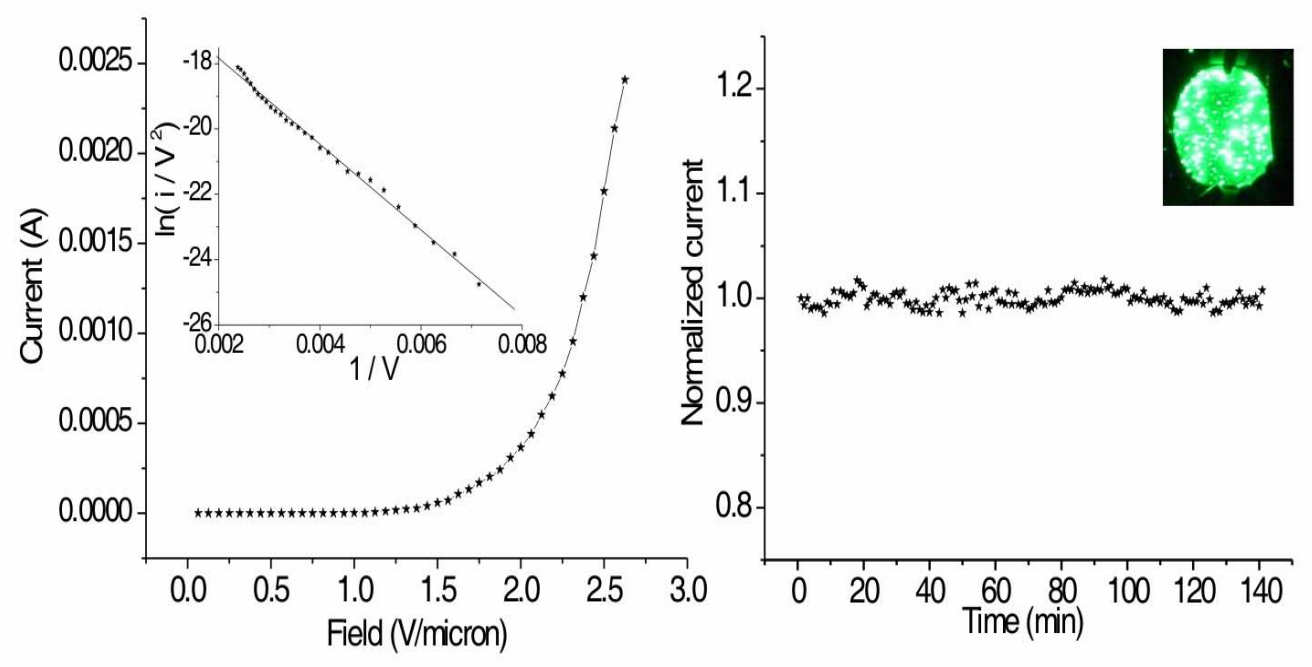

Figure 4.10 (a) I-V plot of thin-MWNTs, (inset) F-N plot (b) normalized emission current as a function of time measured at $400 \mathrm{~V}$ (inset) emission image. 
Field emission measurements were performed in a vacuum chamber under a pressure of $1.0 \times 10^{-6}$ Torr and indium-tin oxide quartz screen printed with conventional green phosphor paste was used as anode. Without MCP between anode and cathode (vacuum gap: $160 \mu \mathrm{m}$ ) the turn-on voltage was observed at $160 \mathrm{~V}$ which corresponds to applied field of $1 \mathrm{~V} / \mu \mathrm{m}$ (figure 4.10a). Emission measurements were analyzed using well known Fowler-Nordheim (F-N) equation

$$
I=\left(a A \beta^{2} E^{2} / \phi\right) \exp \left(-b \phi^{3 / 2} / \beta E\right)
$$

Where $a=1.54 \times 10^{-6} \mathrm{~A} \mathrm{eV} V^{-2}$ and $b=6.83 \times 10^{7} \mathrm{eV}^{3 / 2} \mathrm{~V} \mathrm{~cm}^{-1}$, respectively. $A$ is emission area, $\beta$ is enhancement factor, $E$ is applied electric field in $V \mathrm{~cm}^{-1}$ and $\phi$ is work function in $\mathrm{eV}$ [5]. Local electric field $\left(E_{l}\right)$ at emitter tip can be related to $\beta$ and macroscopic field $\left(E_{m}\right)$ (applied to anode) by

$$
E_{l}=\beta E_{m}
$$

Field enhancement factor can be calculated from the slope of F-N plot $\left(\ln I / V^{2} v s I / V\right)$ assuming $\phi$ as $5 \mathrm{eV}$ similar to graphite.

$$
\text { Slope }=-b \phi^{3 / 2} d \beta^{-1}
$$

Where $\mathrm{d}$ is inter electrode distance The calculated value of field enhancement factor was $\sim 9300$, which implies that $E_{l}$ where nanotubes are emitting electrons is $\sim 9300$ times stronger than $E_{m}$. Value of $\beta$ obtained in this work is much higher than previously reported for MWNTs, ranging from 1000 to 5000 [98-100].The previously reported diameters of MWNTs were in the range of $20-50 \mathrm{~nm}$, therefore the higher value obtained in this study can be attributed to the structure of the nanotubes which have a smaller diameter $(\sim 10 \mathrm{~nm})$ and higher aspect ratio $(\sim 1500)$. Figure $4.10 \mathrm{~b}$ represents the stability 
study of electron emission which had an average fluctuation of less than $2 \%$, measured for 140 minutes at a DC bias of $400 \mathrm{~V}$. Inset of figure $4.10 \mathrm{~b}$ illustrates fairly uniform emission image obtained at $6.25 \mathrm{~V} / \mu \mathrm{m}$ with a $1 \mathrm{kHz}$ sine wave. Hotspots in the emission image can be attributed to the uneven length of nanotubes.

Electron multiplication was characterized by placing a commercial MCP (Hamamatsu) on top of the thin-MWNTs cathode. FE measurements were first made without MCP (distance between anode and cathode $380 \mu \mathrm{m}$, see figure $4.11 \mathrm{~b}$ ). For this case the turn-on voltage was $550 \mathrm{~V}$ and the calculated field enhancement factor $(\beta)$ was 7800. Then MCP was placed at the same distance of $380 \mu \mathrm{m}$ (from cathode) and experiments were conducted with MCP unbiased and biased, respectively. When MCP was unbiased turn-on voltage was 1975 volts and $\beta$ was $\sim 1000$ and in MCP biased condition $\left(\mathrm{V}_{\mathrm{bot}}=100 \mathrm{~V}\right.$ and $\left.\mathrm{V}_{\text {top }}=500 \mathrm{~V}\right)$ the turn-on voltage was $950 \mathrm{~V}$ and $\beta$ was $\sim 6850$ The decrease in turn-on voltage in biased condition may be attributed to the change in the field concentration and significant increase in $\beta$. The total current at an applied voltage of $1500 \mathrm{~V}$ in MCP biased condition was $1.2 \mathrm{~mA}$ and without MCP was $0.16 \mathrm{~mA}$. The 7.5 times higher current with MCP is attributed to electron multiplication. So by placing an MCP on top of CNT emitters higher emission current could be achieved at moderate conditions. This unique approach could enable more consistent and reliable performance of CNT emitters operating at less power and enhanced life. Figure 4.11a illustrates emission measurements with and without MCP. The inset of figure 4.11a shows the emission image with well defined emission spots that are due to focused electrons as MCP channels prevent electron spreading. The commercial MCP plate placed on top CNTs amplified current by few micro amperes. However they are not suitable for 
achieving higher currents (few amperes) because of the lower secondary emission properties of the MCP. In section 4.4 the fabrication and of a new ceramic MCP with stable and high secondary emission materials and capable of achieving higher amplification will be discussed.

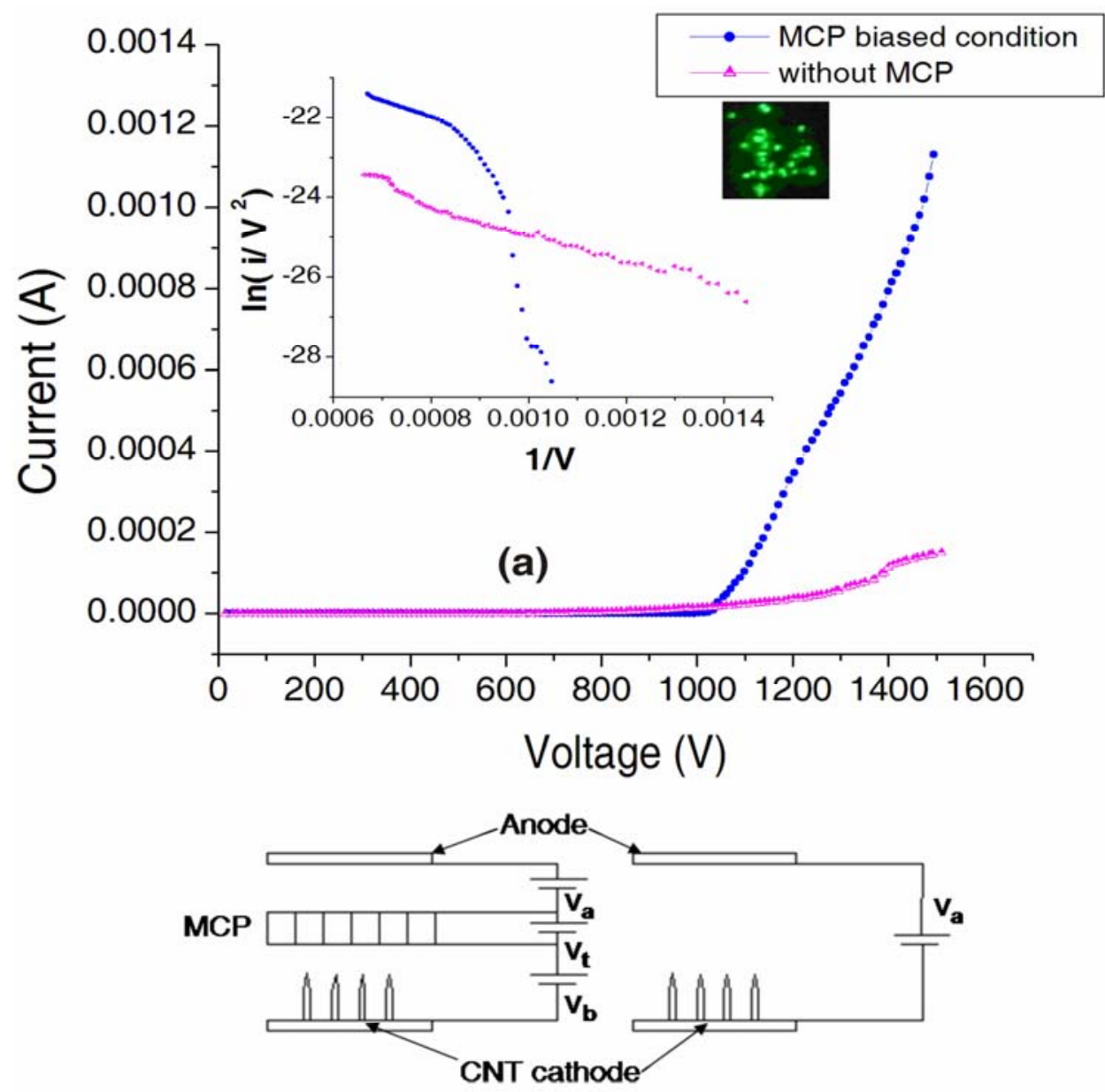

(b)

Figure 4.11 (a) Emission current vs. applied voltage plot of thin-MWNTs in MCP biased condition and without MCP (inset FN plot \& emission image with well defined spots, $V_{a}=1500, V_{b o t}=100, \& V_{t o p}=500 \mathrm{~V}$ ) (b) schematic of measurement circuit. Distance between anode-cathode (right) \& distance between MCP-cathode (left) is $380 \mu \mathrm{m}$. 


\subsubsection{SYNTHESIS AND FIELD EMISSION CHARACTERIZATION OF TUNGSTEN OXIDE MULTISTAGE EMITTERS}

Figure 4.12a shows the SEM image of tungsten oxide nanowires grown on electrochemically sharpened tungsten tip. The two-stage emitter geometry i.e. nanowires on tungsten tip can be clearly observed from the image. The diameter and length of the sharpened tungsten tip were $\sim 1.7 \mu \mathrm{m}$ and $10000 \mu \mathrm{m}$ respectively. The average length of the nanowires was about $250 \mathrm{~nm}$ and diameters were in the range of 10-25 $\mathrm{nm}$. Figure 4.12b shows the TEM image, from which the inter-planar spacing (d) was determined using the two-dimensional Fourier transform. The $d$ value was measured to be $3.81 \AA$ which corresponds to the (010) plane of $\mathrm{W}_{18} \mathrm{O}_{49}\left(\mathrm{WO}_{2.72}\right)$ (Monoclinic, P2/m, JCPDS card number 84-1516). From the TEM image, growth direction of the synthesized nanowires was observed to be [010]. The growth direction was explained by Frey et al. by using Bravais-Friedel-Donnay-Harker (BFDH) law, which states that growth rate of a crystal is inversely proportional to the inter-planar spacing of that face, which is the short axis i.e. (010) plane for $\mathrm{W}_{18} \mathrm{O}_{49}[101]$.

The growth mechanism of the tungsten oxide nanowires on tungsten substrates or sharpened tips is suggested as either vapor-solid process [32-33] or chemically induced strain [77], but the detail mechanism is not well understood. According to the chemically induced strain mechanism, the nanowires are grown by the conversion of strained oxide at the $\mathrm{W} / \mathrm{WO}_{\mathrm{x}}$ interface to unstrained oxide in nanowire. Some authors assume that the nanowire growth is governed by vapor-solid process since there is no catalyst involved in the growth. Oxygen source for the growth of nanowires comes from the native oxide 
layer. This might have been formed due to the oxidation of tungsten surface during the heating phase of the three-step growth process.

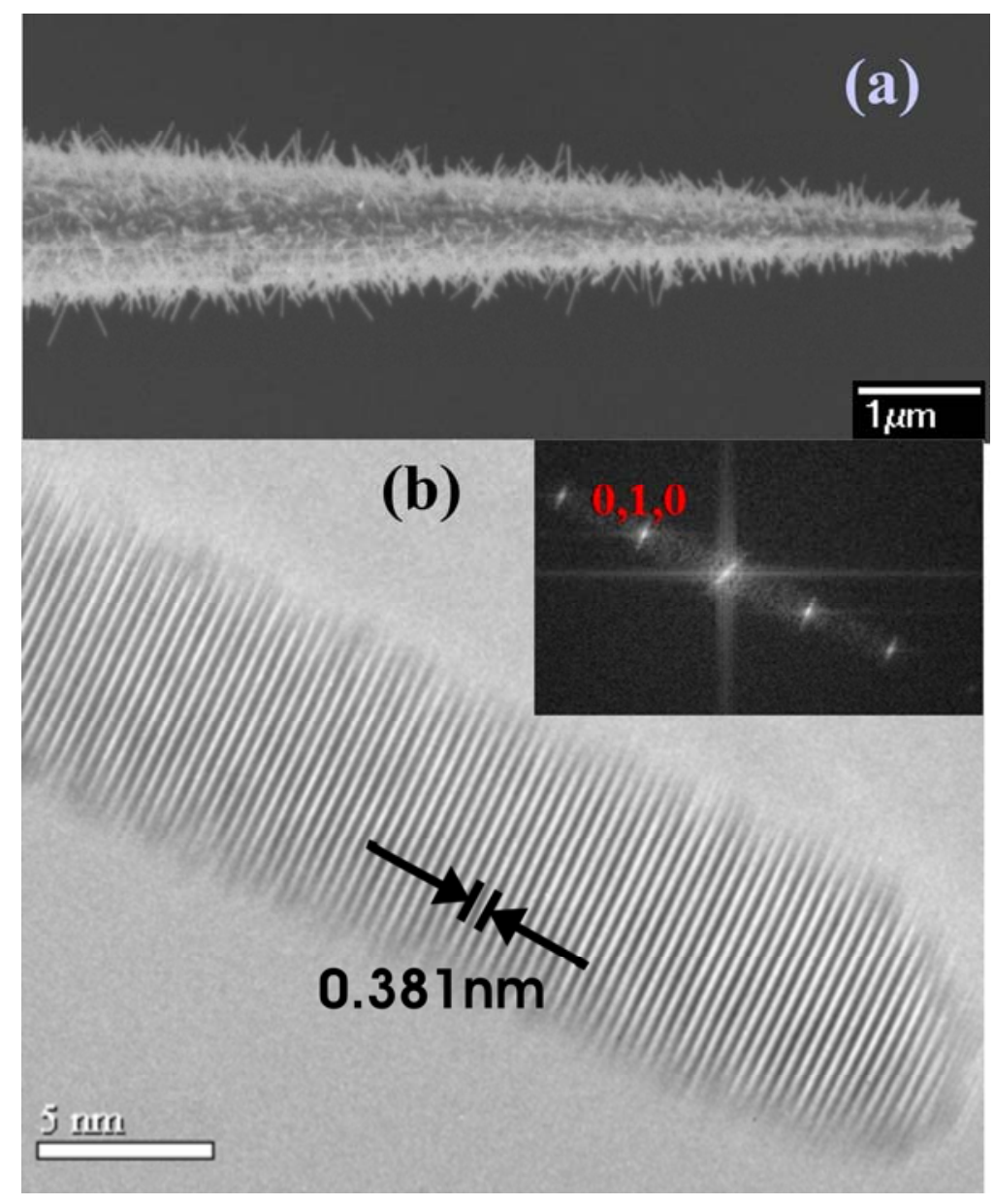

Figure 4.12 (a) SEM image of tungsten oxide nanowires grown on electrochemically etched tungsten tip (two-stage emitter geometry) (b) High magnification TEM image of the $\mathrm{W}_{18} \mathrm{O}_{49}$ nanowires (Monoclinic, $\mathrm{P} 2 / \mathrm{m}$, JCPDS card number 84-1516), inset shows the two dimensional Fourier transform pattern of the TEM image.

Figure 4.13a shows the field emission characteristic of the two-stage emitter i.e. tungsten oxide nanowires on tungsten tip, measured at $1 \times 10^{-6}$ Torr and figure 4.13b,c 
show the schematic of emitter geometry. It was observed that the threshold field for emission was $\sim 0.95 \mathrm{~V} / \mu \mathrm{m}$ which is lower than the previously reported data [29-34] and the electric current obtained was as high as $170 \mu \mathrm{A}$ per tip. Threshold field is the applied electric field required to extract an emission current of few nano amperes from the sample. The dependence of threshold field and field enhancement factor on the interelectrode distance $(D)$ for a non planar emitter has been reported by Smith et al. It was explained that the threshold field for emission decreases as the electrode separation increases, when D is less than the height of the emitter, $h$. In the present study $h$ corresponds to the effective length of the emitter i.e. sum of nanowire and electrochemically sharpened tungsten tip lengths. However for a sufficiently large electrode separation $(D>>h)$ the threshold field was observed to reach an asymptotic value [102]. In this study the observed low threshold field for emission might be either due to the shorter inter-electrode distance of $1150 \mu \mathrm{m}$ which is less than the emitter height, or the multistage field enhancement effect. Further studies are required to clearly understand the cause for lower threshold field. Emission measurements were analyzed using Fowler-Nordheim (F-N) equation 4.4. Field enhancement factor was calculated from the slope of F-N plot $\left(\ln I / V^{2} v s I / V\right)$ assuming $\phi$ as $5.7 \mathrm{eV}$ similar to $\mathrm{WO}_{3}$. Inset of figure 4.13 shows the F-N plot, which can be divided into three regions R1: linear current voltage dependence, R2: slow current variation (saturation region), R3: rapid rise of voltage. The calculated $\beta$ values for these three regions were 2900, 19800 and 3500 respectively. 

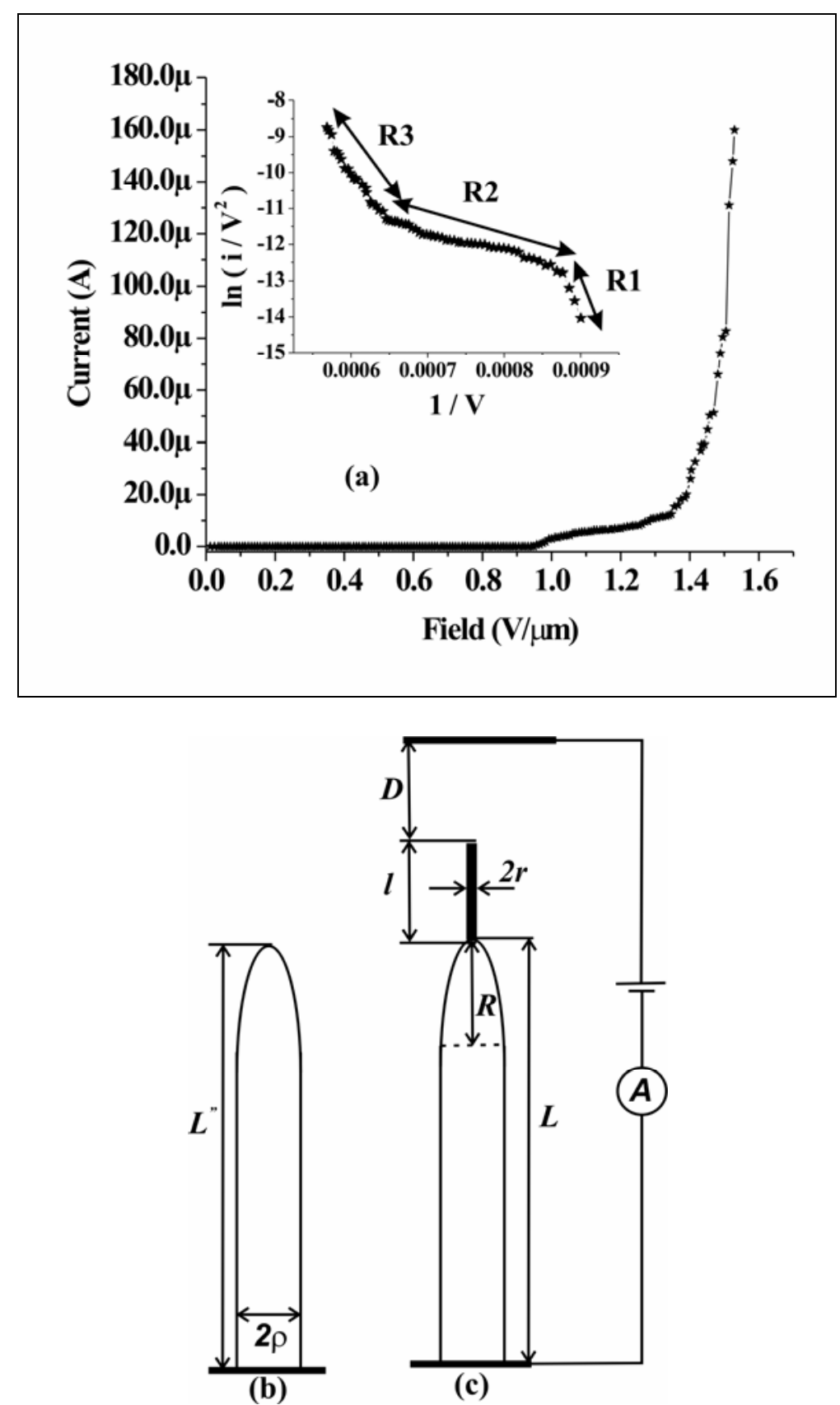

Figure 4.13 (a) Field emission measurements i.e. field vs. current of tungsten oxide multistage (two-stage) field emitter and its corresponding Fowler-Nordheim plot shown in inset. R1: linear current voltage dependence, R2: slow current variation (saturation region), R3: rapid rise of voltage region, respectively. Field enhancement factor $(\beta)$ for these three regions $\sim 2900,19800$ and 3500 respectively (b) Schematic of hemi-ellipsoid 
on a plane geometry of the electrochemically sharpened tungsten tip (geometrical/mathematical model), where $L^{\prime \prime}$ is the semi-major axis length and $\rho$ is the semi-minor axis length (c) Schematic of experimental configuration and a simple model of two-stage emitter, where $l$ is the length and $r$ is radius of the nanowire. $L$ is the length and $\mathrm{R}$ is the radius of electrochemically sharpened tungsten tip.

The calculated $\beta$ value of sharp tungsten tip considering the hemi-ellipsoid on a plane geometry [56] is given by

$$
\beta=\zeta^{3} /[\{v \ln (v+\zeta)\}-\zeta]
$$

$$
\begin{aligned}
& \zeta=\left(v^{2}-1\right)^{1 / 2} \\
& v=L^{\prime \prime} / \rho
\end{aligned}
$$

$L^{\prime \prime}$ is the semi-major axis length and $\rho$ is the semi-minor axis length shown in figure $2 \mathrm{~b}$. In this study $L^{\prime \prime}$ is $\sim 10000 \mu \mathrm{m}$ and $\rho$ is $\sim 80 \mu \mathrm{m}$ and $\beta$ without nanowires corresponds to $\sim 3450$. The $\beta$ for a two-stage field emitter i.e. tungsten oxide nanowires on tungsten tip is given by

$$
\beta_{t o t}=\beta_{n w} \beta_{t i p}=\frac{l}{(l+D)}\left(1+\frac{D}{r}\right) \frac{h}{(h+D)}\left(1+\frac{D}{R^{\prime}}\right)
$$

Where $l, r$ are the length and radius of the nanowire, while $L$ is the length, $R$ is the radius of electrochemically sharpened tungsten tip and $\mathrm{D}$ is the inter-electrode distance. $R^{\prime}=R+l$ and $h=L+l$ are effective radius and length of the emitter i.e. nanowire and electrochemically sharpened tungsten tip [57]. For $l \sim 0.2, \quad r \sim 0.01, \quad h \sim 10000.2$ and $R^{\prime} \sim 1.025$, all dimensions in $\mu m$, the calculated $\beta$ value is $\sim 20100$. So the experimentally 
determined value of $\beta \sim 19800$ obtained here can be attributed to the multistage field enhancement effect of the two-stage emitter geometry. The current saturation observed in R2 of F-N plot was explained by Fursey et al [37]. The current saturation is dependent on several inter-related processes, which reduce the electron concentration in the nearsurface region of the semiconductor, increase the field penetration into the sample, change the field geometry near the emitter and increase the voltage drop across the crystal bulk. Under these conditions the emission current has been suggested to be limited by the supply of carriers to the surface and somewhat dependent on the applied potential up to the abrupt rise of carrier concentration in R3 [37].

Figure 4.14a shows the field emission measurements of the two-stage emitter at various vacuum conditions ranging from $1 \times 10^{-6}$ to $3 \times 10^{-3}$ Torr and figure $4.14 \mathrm{~b}$ the emission recovery characteristics. In a study performed on $\mathrm{ZnO}$ nanorods by Kim [22], emission was limited up to $10^{-4}$ Torr. In another study by Tondare [23] on tungsten emitters, emission was limited to few nano amperes at $10^{-3}$ Torr. While the two-stage field emitters in the present study have demonstrated high emission current of a few $\mu \mathrm{A}$ under various vacuum conditions. It can be observed from figure $3 a$ that these emitters have better performance in relatively poor vacuum compared to other reported emitters [22-23]. As with any emitter operated in commercial environments, the ability to withstand oxygen ambient can be crucial. Oxide materials have relatively high melting temperature and have been observed to be stable in oxygen and poor vacuum conditions. This makes these oxide emitters an alternate candidate suitable for stable field emission in low vacuum conditions. The emission recovery measurements showed that, emission was recoverable after measurements at $10^{-3}$ Torr as shown in figure $4.14 \mathrm{~b}$. However, the 
threshold voltage for emission was observed to be increased. One of the possible reasons could be the adsorption of foreign atoms at $10^{-3}$ Torr which might have still remained on the emitter even after returning to high vacuum i.e. $10^{-6}$ Torr. Short-term DC emission stability measurements at $10^{-6}$ Torr showed an average current fluctuation of $10 \%$, which is fairly stable. Better emission stability is required for device application; methods for achieving this have to be further investigated. Long-term emission stability under various vacuum conditions is also needs further investigation. The emitter performance, in terms of threshold voltage and emission current can be further improved by fabricating an electrochemically sharpened tungsten tip with a narrower diameter i.e. in the range of few $\mathrm{nm}$. Moreover, if the spacing of the nanowire-to-nanowire is increased, screening effect [52] will be decreased, which will also increase the emitter performance. 


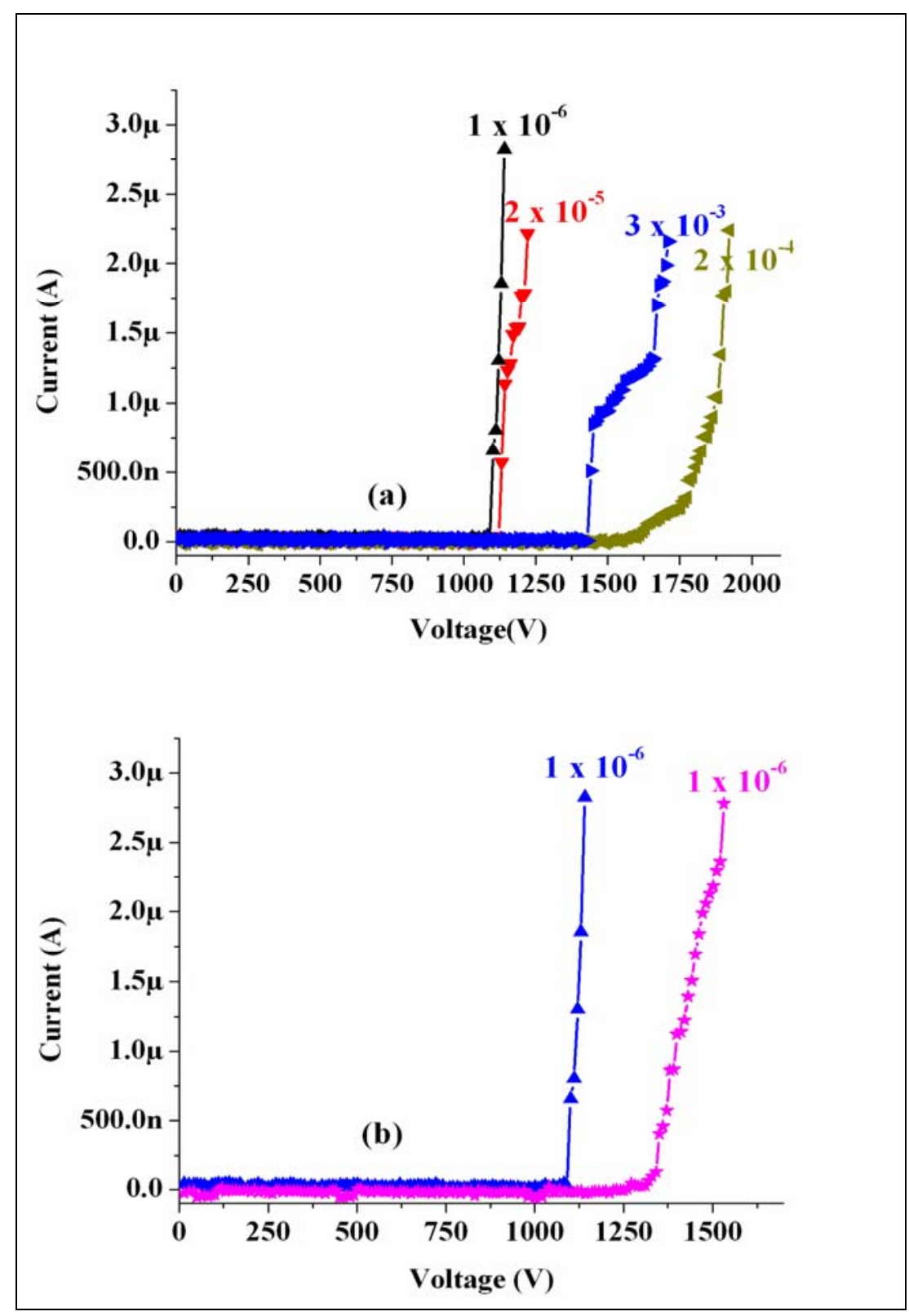

Figure 4.14 (a) Field emission behavior of tungsten oxide multistage (two-stage) field emitter under various vacuum conditions i.e. $10^{-6}-10^{-3}$ Torr (b) Solid triangles indicate measurements made at $10^{-6}$ Torr and Solid stars indicate emission recovery measurement at $10^{-6}$ Torr after measurements at $10^{-3}$ Torr. 


\subsubsection{SYNTHESIS AND FIELD EMISSION CHARACTERIZATION OF CNT MULTISTAGE PILLAR ARRAYS}

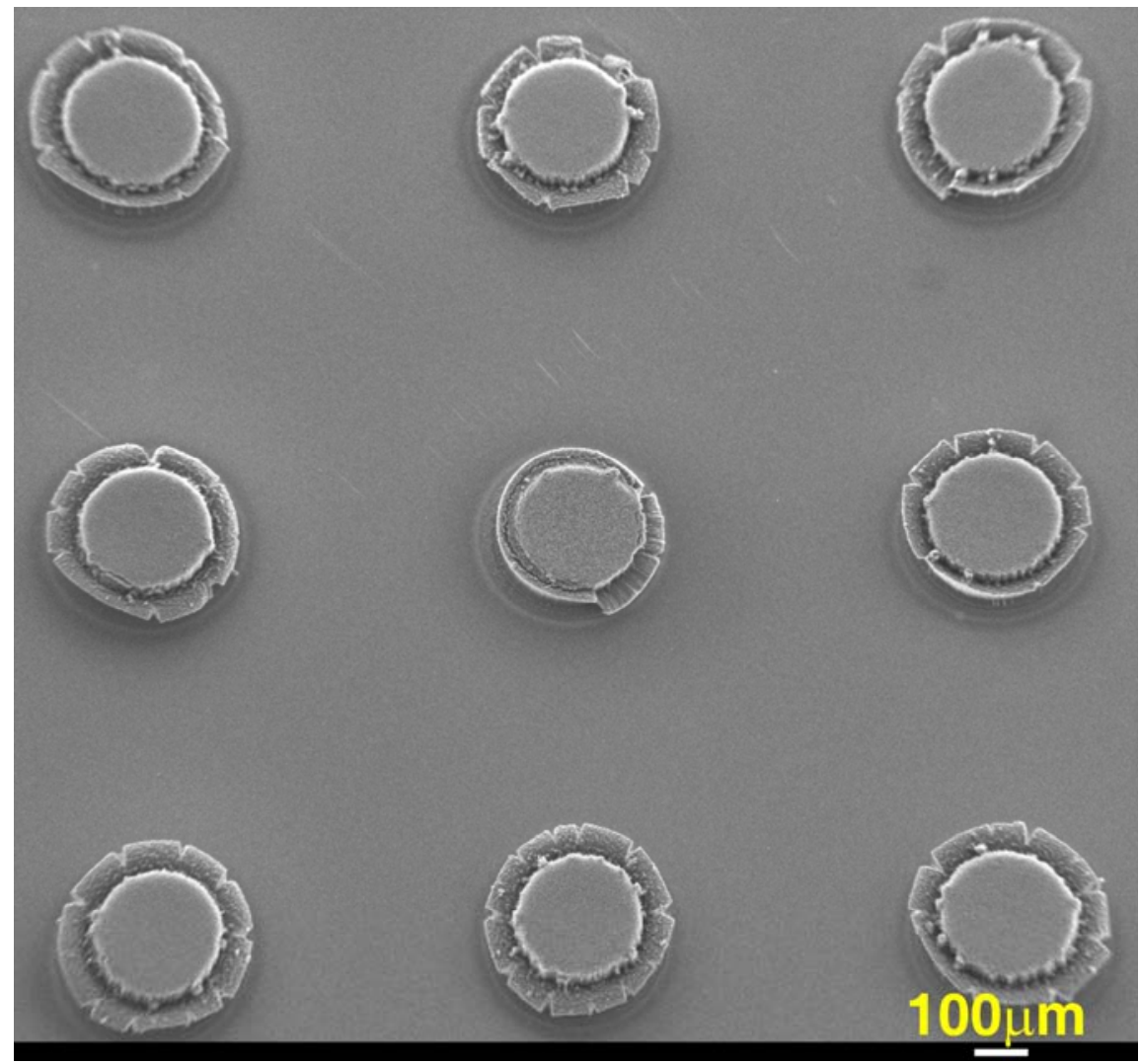

Figure 4.15 Aligned carbon nanotube arrays grown on porous silicon.

Figure 4.15 shows the SEM image of vertically aligned carbon nanotube pillar arrays. These arrays were grown on porous silicon (Si) [103], which assisted in the alignment of the nanotubes. The location of the pillars on porous-Si was controlled by depositing the catalyst through a ceramic mask which had the similar dimensions of the MCP. This fabrication step was helpful in aligning the field emitters with MCP during the emission enhancement experiments. Figure 4.16a shows the SEM image of vertically grown MWNTs on porous-Si nanotemplate. 

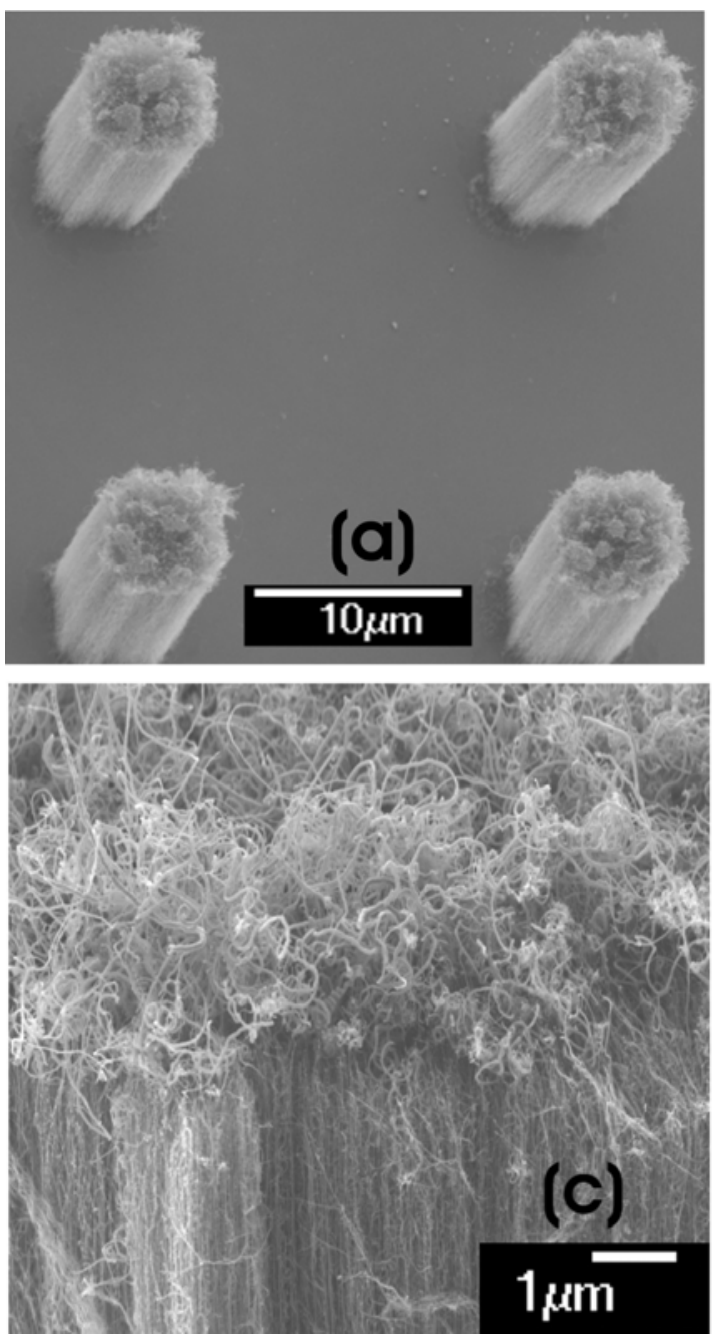

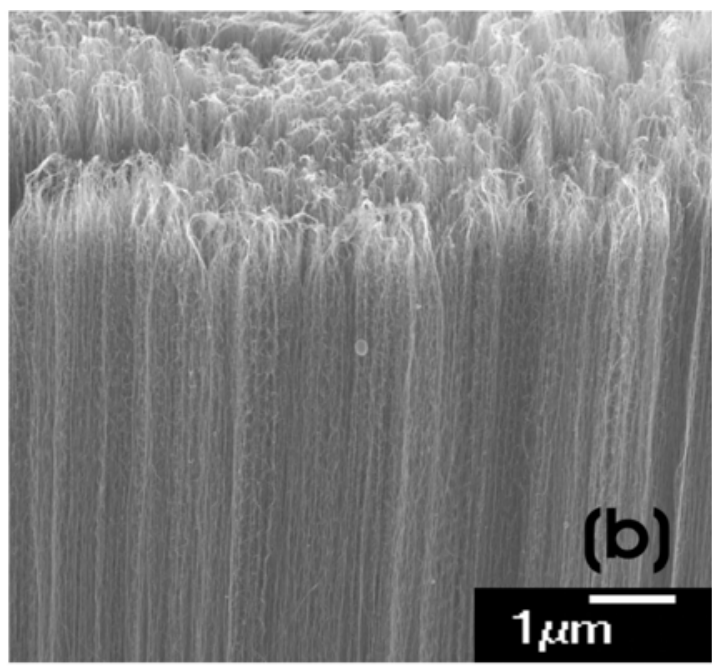

Anode

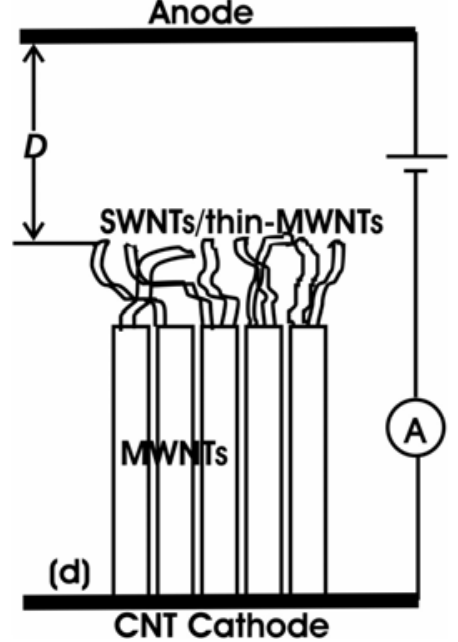

Figure 4.16 SEM images of (a) Vertically aligned/oriented MWNT arrays grown on porous silicon (b) plasma etched MWNTs (c) carbon nanotube multistage structure i.e. thin-MWNT and SWNT on top of MWNT (d) Schematic of the multistage structure and field emission measurement schematic.

The growth mode of nanotubes on the porous-silicon is base growth mode similar to the one achieved by Fan et al [48]. This was verified by completely removing the nanotubes by reactive ion etching on several samples. Nanotube growth was observed on the resulting substrates when the normal growth process was followed. Vertical 
alignment for nanotubes grown on porous-Si nano-templates is achieved by van der Walls force interaction between the adjacent nanotubes forming bundles with high rigidity from densely packed catalyst particles. The rigidity enables nanotubes to keep growing along the original direction i.e. normal to the substrate and even outermost nanotubes are held by inner nanotubes without branching away [48]. Length of the nanotubes was controlled by varying the precursor gas flow time during the CVD growth. The average length and diameter of MWNT arrays grown for 45 min were $\sim 55 \mu \mathrm{m}$ and $\sim 15-20 \mathrm{~nm}$, respectively.

The multistage structure/geometry of the CNTs seen in the SEM image of figure $4.16 \mathrm{c}$ and schematic diagram, figure $4.16 \mathrm{~d}$ can be distinguished by comparing with the top surface of $\mathrm{O}_{2}$ plasma etched MWNTs shown in figure $1 \mathrm{~b}$. The presence of smaller diameter nanotubes i.e. SWNT and thin-MWNT on top of the MWNT arrays i.e. multistage structure was determined from TEM and Raman spectroscopy. For TEM analysis few drops of suspension obtained from nanotube blocks sonicated in dichloroethane, were placed onto a TEM grid. Figure 4.17a, b show the TEM images consisting of MWNT and thin-MWNT. The defective structure of thin-MWNT seen in figure $4.17 \mathrm{~b}$ may be caused by lower growth time of $\sim 5 \min$ [5]. From the TEM analysis presence of SWNT was not found this may be due the modest amount of SWNT existence in the total sample, however their presence was known from Raman spectroscopy. Usually radial breathing mode (RBM) peaks are not observed in MWNTs $[5,105]$, however in our samples they were observed due to the presence of SWNT and thin-MWNT on top of MWNTs (figure 4.16c, d). The diameters $\left(d_{t}\right)$ of nanotubes were calculated from the relation $d_{t}=248 / \omega_{R B M}$. The $d_{t}$ values ranging from $\sim 2.76-3.52 \mathrm{~nm}$ 
calculated for $\omega_{\mathrm{RBM}} 50-150 \mathrm{~cm}^{-1}$ correspond to thin-MWNTs, $d_{t}$ values ranging from $\sim 1.28-1.96 \mathrm{~nm}$ for $\omega_{\mathrm{RBM}} 125-200 \mathrm{~cm}^{-1}$ correspond to SWNTs (figure 4.17a-c). The calculated $d_{t}$ of nanotubes for RBM frequencies $\left(\omega_{R B M}\right) 50-200 \mathrm{~cm}^{-1}$ agreed well with internal diameters obtained from TEM analysis. The $G$ band at $\sim 1570 \mathrm{~cm}^{-1}$ of Raman spectra (figure 4.17c) indicates formation of graphene sheets and $D$-band peak at $\sim 1344$ $\mathrm{cm}^{-1}$ indicates defects or impurities in the sample. The average length and diameter of the SWNT and thin-MWNTs were $\sim 10-15 \mu \mathrm{m}$ and $\sim 2-10 \mathrm{~nm}$, respectively.

FE measurements of as-grown MWNT and multistage arrays at $\sim 1 \times 10^{-6}$ Torr are shown in figure 3. Obtained data was analyzed by Fowler-Nordheim (FN) equation. The turn-on field $\left(E_{t}\right)$ and current at a field of $1 \mathrm{~V} / \mu \mathrm{m}$ as seen in figure 4.18 for the multistage and MWNT arrays was $\sim 0.4 \mathrm{~V} / \mu \mathrm{m}, 0.6 \mathrm{~V} / \mu \mathrm{m}$ and $\sim 450 \mu \mathrm{A}, 14 \mu \mathrm{A}$ respectively. The lower turn-on field ( $\sim 1.5$ times) and higher emission current ( $\sim 32$ times) for multistage arrays can be attributed to its geometry, i.e. to higher field enhancement at the smallest nanotube tip on the vertically aligned MWNTs. The calculated $\beta$ value from the slope of FN plot $\left(-b \phi^{3 / 2} D \beta^{-1}\right)$ assuming $\phi 5 \mathrm{eV}$ similar to graphite was $\sim 26200$ and $\sim 8400$ for multistage and as-grown MWNT arrays, respectively. The higher $\beta(\sim 3$ times) value may also have resulted in higher field emission in multistage MWNT arrays [57, 105]. 

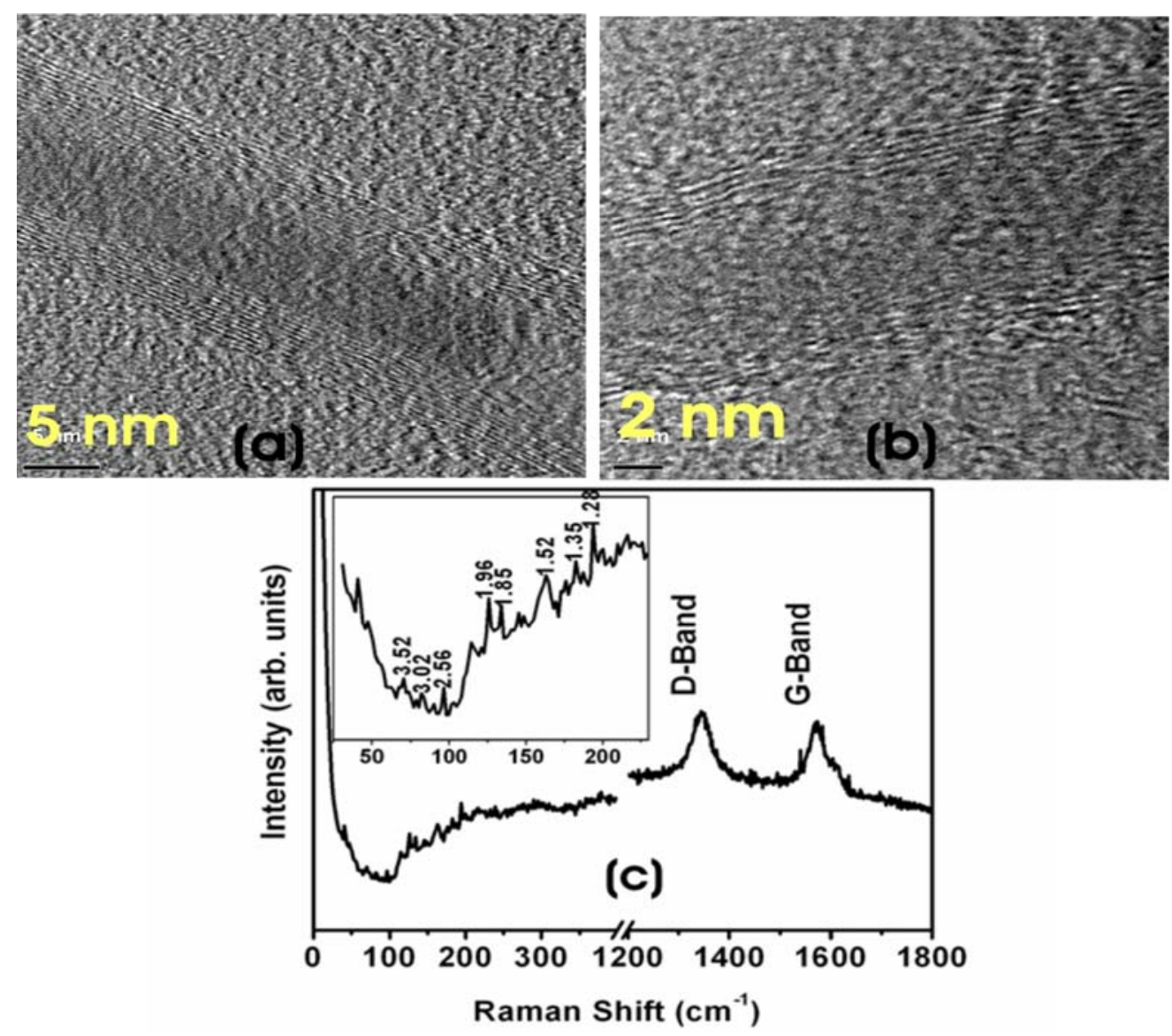

Figure 4.17 HRTEM images of (a) MWNT and (b) thin-MWNT (c) Raman spectrum ( $\lambda$ : $514 \mathrm{~nm}$ ) of multistage CNTs with Argon ion $\left(\mathrm{Ar}^{+}\right)$laser, inset radial breathing mode (RBM) peaks, inset numbers correspond to inner tube diameters of thin-MWNTs and SWNT.

The highest achieved emission current in this study was relatively less, which can be attributed to the p-type porous-Si substrates on which CNTs were grown. Previous reports have shown that CNTs grown on p-type Si substrates showed lower emission current which was explained on the basis of interface between silicon substrate and carbon nanotube. It is due to, the availability of electrons undergoing tunneling from CNTs, which depends on the efficiency of electron injection mechanism from substrate 
across the nanotube interface. The CNT-Si interface was treated as a metalsemiconductor interface and Schottky contact barrier was possible formed. Since field emission is measured by applying a negative bias to CNT substrate, p-type Si and CNT combination was considered as a reverse-biased Schottky junction thus increasing effective built-in potential [106]. In this work p-type substrates were chosen due to the ease of porous-Si fabrication. Further studies have to be performed with the CNTs grown on n-type porous-Si to achieve higher current. This efficient emitter design with enhanced field emission properties can be used in future micro-vacuum electronic devices

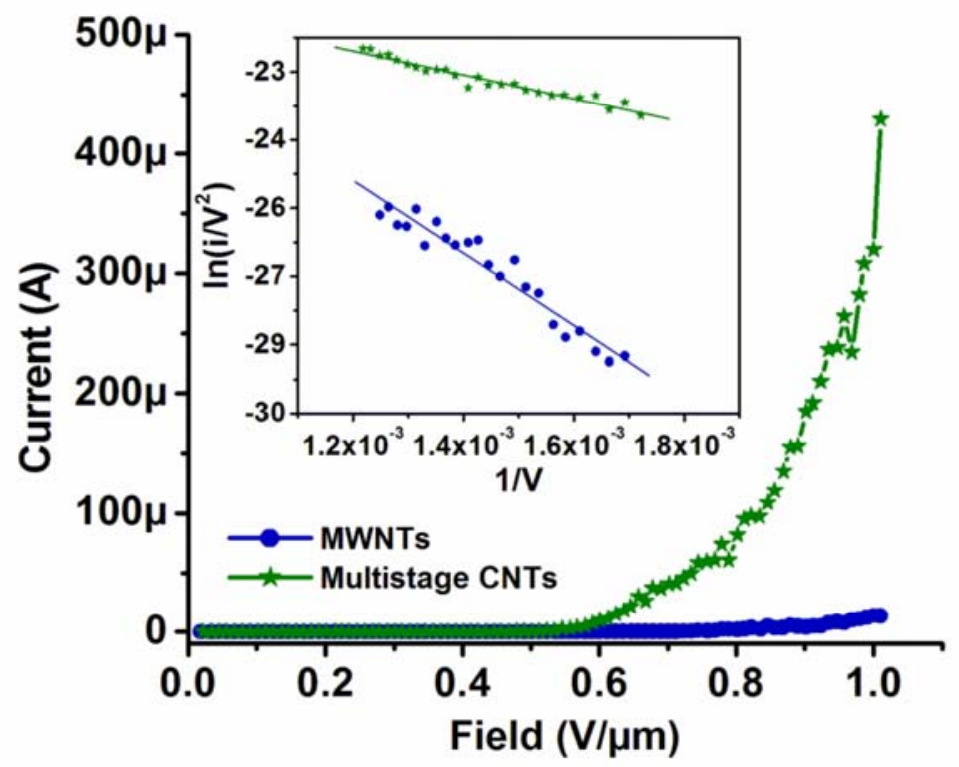

Figure 4.18 Field emission plot i.e. emission current vs. applied field, for multistage and only MWNT arrays, turn-on field $\left(E_{t}\right)$ was $\sim 1.5$ times lower and emission current was $\sim 32$ times higher for multistage in comparison to only MWNT arrays, inset corresponding FN plot. 


\subsection{FABRICATION AND AMPLIFICATION CHARECTERIZATION OF A NEW MICROCHANNEL BASED ON HIGH SECONDARY YIELD MgO-COVERD \\ CNT}
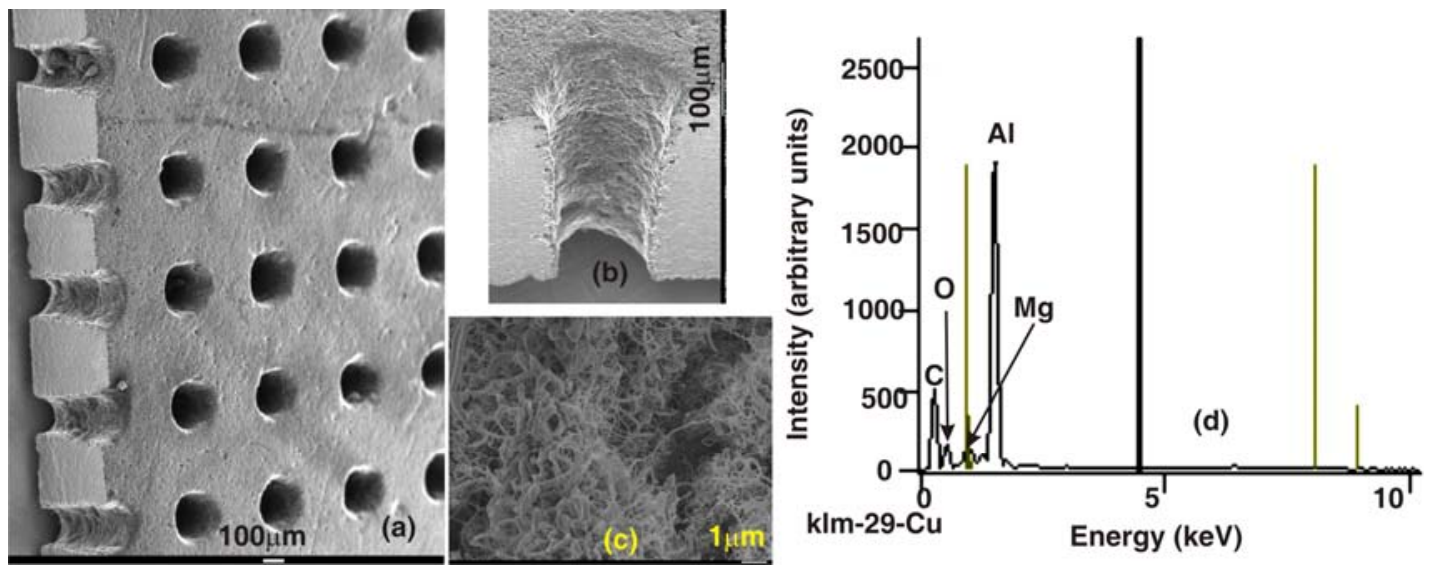

Figure 4.19. $\mathrm{SEM}$ image of (a) Ceramic $\left(\mathrm{Al}_{2} \mathrm{O}_{3}\right)$ microchannel plate (MCP) with $\mathrm{MgO}$ -

coated CNTs (b) crossection of MCP channel showing uniform layer of MgO-coated

CNTs (c) magnified view of MgO-coated CNTs along the channel and (d) representative

EDX spectrum of one of the several ponits taken along the channel with $\mathrm{MgO}$-coated

CNTs showing the presence of $\mathrm{Mg}, \mathrm{O}, \mathrm{C}$ and $\mathrm{Al}$.

This sections discusses the fabrication of a new ceramic microchannel plate based on a high secondary yield MgO-covered CNTs. Figure 4.19a illustrates the SEM image of the MCP and 4.19b shows the channel cross-section with uniform layer of $\mathrm{MgO}$-coated CNTs. Figure $4.19 \mathrm{c}$ shows the $\mathrm{MgO}$ covered nanotubes inside the channel and figure 4.19d shows the representative image of the EDX spectrum i.e. of one point along the channel. From the elemental analysis the presence of $\mathrm{Mg}, \mathrm{O}, \mathrm{C}$ and $\mathrm{Al}$ was noticed thus providing the evidence that $\mathrm{MgO}$ was coated inside the channel. 

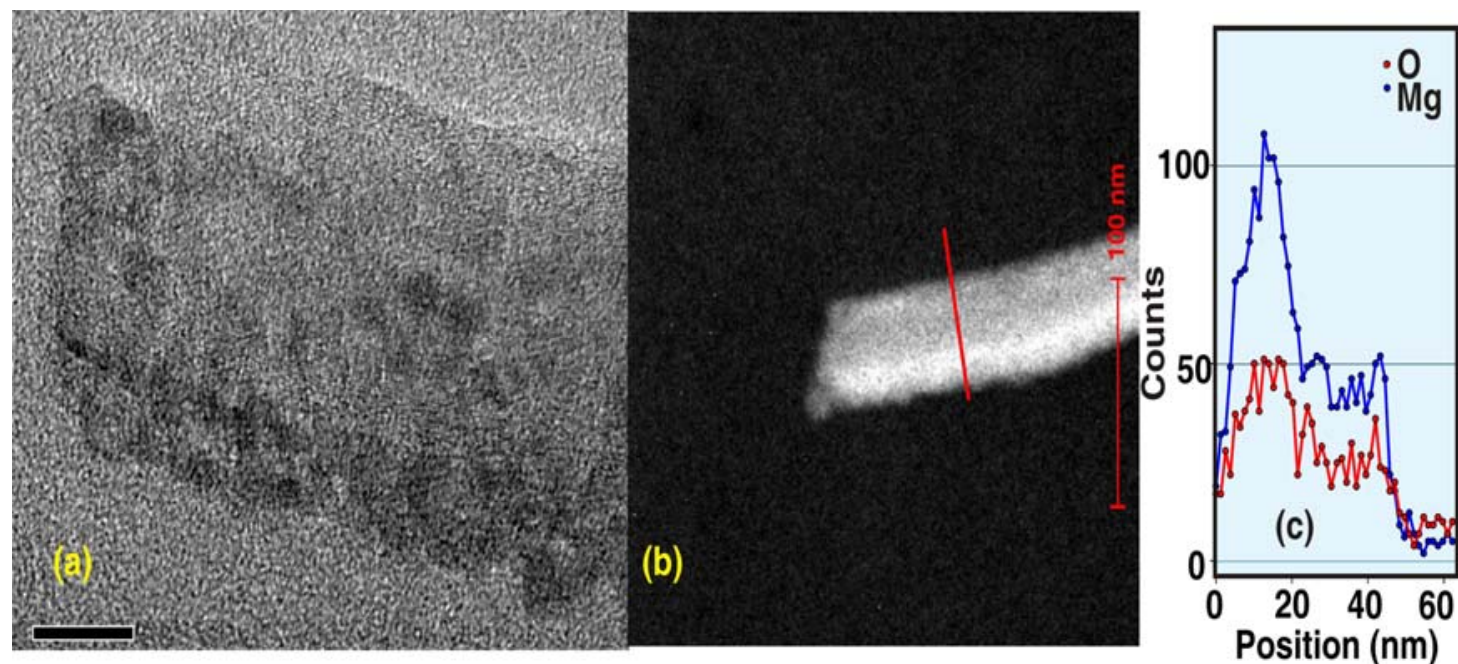

Figure 4.20 (a) HRTEM image of MgO-covered CNT, higher amount of MgO coating

can be seen on side of the CNT from the image; which might be the nanotube surface facing away from the channel surface (b) high angle annular dark-field (HAADF) image of $\mathrm{MgO}$-covered CNT (shown in figure 2a) acquired for 120 minutes (c) EDX spectrum i.e. line scan along the $\mathrm{MgO}$-covered $\mathrm{CNT}$ showing the presence of $\mathrm{MgO}$, higher amount of $\mathrm{MgO}$ coating on one side of nanotube can also be observed from the scan.

The fabricated MCP which is shown in figure 4.19a had an array size of $21 \times 21$ i.e. area of $0.5 \times 0.5$ sq-inch. Channel diameters and length were $\sim 240 \mu \mathrm{m}, 500 \mu \mathrm{m}$ respectively, and the pitch i.e. center to center distance between each channel was $600 \mu \mathrm{m}$. These dimensions were the least feasible with the currently available laser facility in our lab. The TEM image of the nanotubes covered with $\mathrm{MgO}$ is shown in figure $4.20 \mathrm{a}$; the sample for analysis was prepared by scraping off the nanotubes from the channel and the surface. It can be observed from the image that the coating is achieved only on one side which might had been the top surface for the nanotubes grown along the channel. This is expected for nanotubes grown horizontally on a surface, in this case the 
side which does not have any $\mathrm{MgO}$ coating might had been the surface which is facing towards the channel. The EDX results from TEM analysis i.e. line scan along the surface of the nanotube (figure 4.20b) are as seen in figure 4.20c conforming the presence of $\mathrm{MgO}$ coating on the nanotube. Higher amount of coating on the left side can also be observed from the image. The length and diameter of the nanotubes on the MCP were $\sim 40-50 \mu \mathrm{m}$ and $\sim 20-30 \mathrm{~nm}$, respectively. The $\mathrm{MgO}$ thickness varied from $\sim 10-30 \mathrm{~nm}$ with 10-15 $\mathrm{nm}$ for CNTs inside the channel and $30 \mathrm{~nm}$ for the ones on the surface and the ones at channel entrance. SEM and TEM measurements were made on several samples and most of them agreed statistically with results shown in the images here.
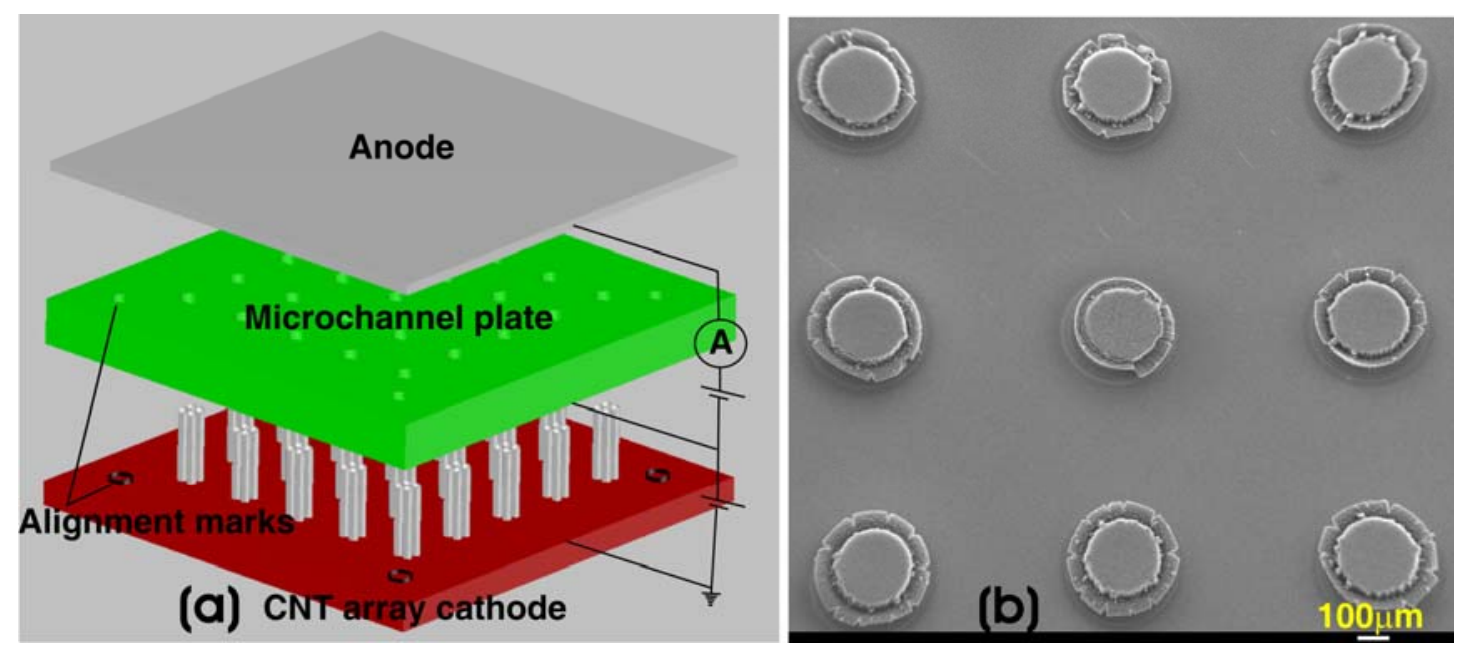

Figure 4.21 (a) Schematic of the CNT and MCP cold electron source (b) CNT emitters.

The fabricated MCP was characterized for its multiplication, by inserting between the nanotube array cathode (figure 4.21b) and anode as represented by the drawing in figure 4.21a. The characterization was achieved by measuring the I-V data and by electron emission imaging. Schematic of the measurement circuit with and without MCP is shown 
in figure 4.22c. All the experiments were conducted under a DC voltage with highest applied voltage of $2500 \mathrm{~V}$ to anode in experiments without and with MCP. For electron multiplication a voltage of $500 \mathrm{~V}$ was applied to MCP bottom face. Figure 4.22a illustrates emission measurements with and without MCP. The total current at an applied voltage of $2500 \mathrm{~V}$ with was $\sim 1.1 \mathrm{~mA}$ and without MCP at $2000 \mathrm{~V}$ was $\sim 0.06 \mathrm{~mA}$. The turnon voltages were approximately same for both the cases (inset of figure 4.22a). Higher current of $0.5 \mathrm{~mA}$ was achievable without MCP around $\sim 2500 \mathrm{~V}$; however it was not stable due to vacuum arcing which led to irreversible damage of CNT emitters. When MCP was placed over the CNT emitters it protected them from vacuum arcing in addition to amplifying the FE current. Higher current with MCP i.e. $\sim 18$ times is attributed to electron multiplication, so by placing an MCP on top of CNT emitter's higher emission current could be achieved at moderate conditions. This unique approach could enable more consistent and reliable performance of CNT emitters operating at less power and enhanced life. Since the MCP had MgO covered CNTs on the surface and the channels FE is also expected from them so we also measured their I-V (figure 4.22a). A current of $0.2 \mu \mathrm{A}$ was observed at a voltage of $\sim 2800 \mathrm{~V}$ from the measurements which is relatively less to the current achieved with a MCP and CNT emitters. This proves that higher current with $\mathrm{MCP}$ is due to electron multiplication, it can be verified by measuring the electron energy spectra of the field emitted electrons which has to be performed in future studies. Figure $4.22 \mathrm{~b}$ shows the emission image with well defined emission spots that are due to focused electrons as MCP channels prevent electron spreading. 

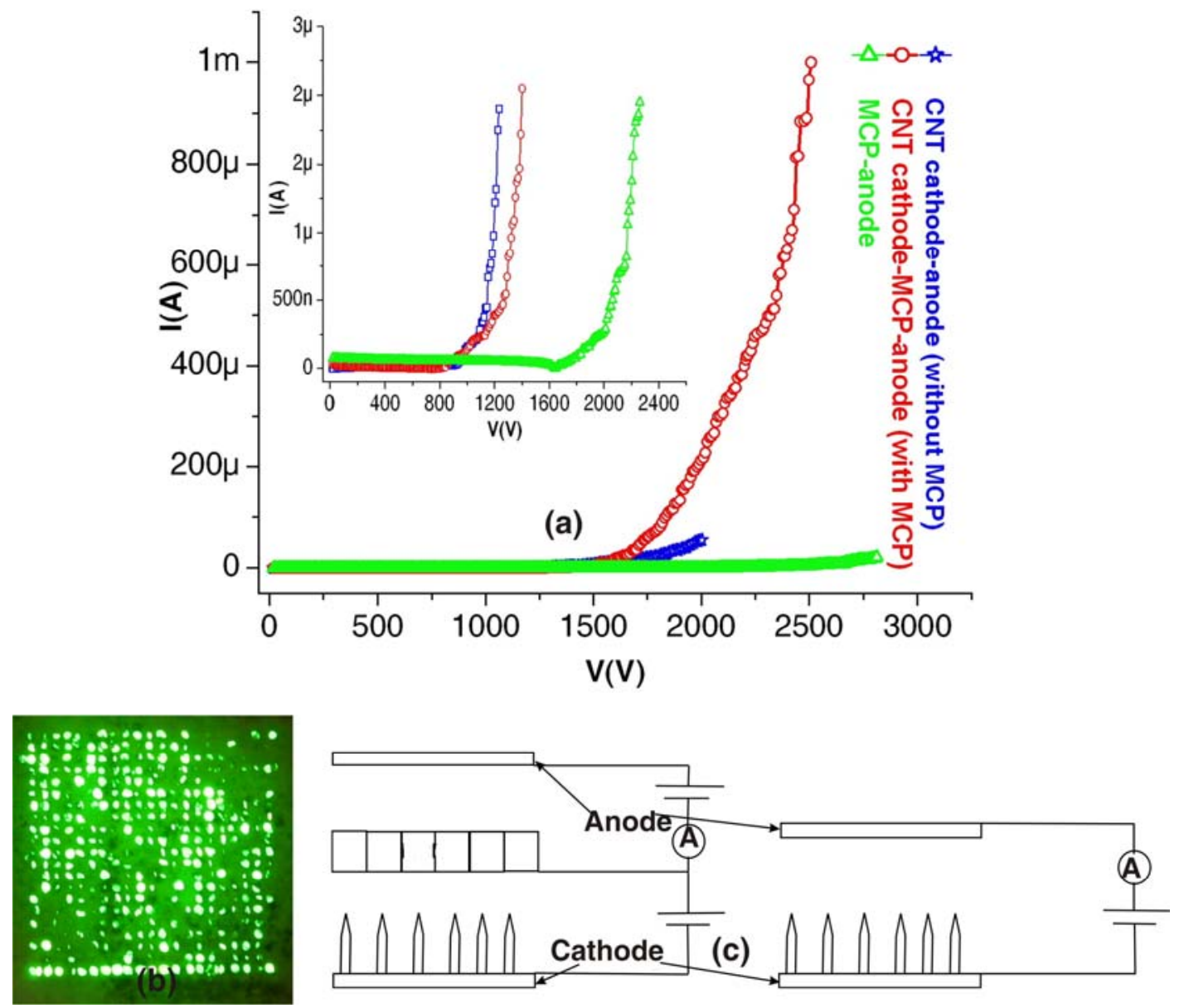

Figure 4.22. (a) Field emission data (I-V) with and without MCP between CNT cathode and anode and only MCP (inset) showing the turn-on voltage (b) bright and focused emission with MCP over the CNT cathode. Increase in brightness is due to amplification of input current by secondary electron generation and well defined spots are due to focusing achieved from MCP channels (c) schematic of measurement circuit with and without MCP.

Even though the exact mechanism of high SEY from MgO-CNT system is not clearly understood, it is assumed as Townsend avalanche [93] that can be explained as follows. Secondary electrons are generated from the $\mathrm{MgO}$ film when primary electrons bombard it. 
Due to the high resistivity of the $\mathrm{MgO}$ film its surface acquires a large positive charge and a high field is created across the film. Subsequent electrons bombarding the film penetrate a certain distance into the film generating more secondaries. Each new secondary electron generates additional electrons by internal ionization resulting in Townsend type avalanche [93]. The higher field may also be due to the geometry of the $\mathrm{MgO}-\mathrm{CNTS}$ i.e. their aspect ratio and nano tip radius, leading to higher number of secondaries and their probability to escape into vacuum. The high SEY yields were only observed from MCP with MgO-CNT and not with the one with only CNT. Similar kind of result was observed by Yi et. al. from their secondary electron emission and electron energy distribution studies of $\mathrm{MgO}-\mathrm{CNT}$ sample. It was concluded from their experiments that high yield can only obtained from $\mathrm{MgO}-\mathrm{CNT}$ and not from CNT or $\mathrm{MgO}$ alone [13-15, 90-92]. Figure 4.23 shows the variation of SEY for various materials from which it can be observed that MgO-CNT has the highest value. The simulation was performed with charged particle optics (CPO) software considering lead glass[13], alumina [13,17-18] and MgO-coated CNTs [90-92] as the emissive layer along the channel. The simulated channel had the same dimensions as the one fabricated one in this work. The simulation results demonstrate that the high yield achieved for the relatively small aspect ratio channels is a result of high secondary emission property of $\mathrm{MgO}$ coated CNTs. In future work to achieve higher current levels several MCP stacked one over the other i.e. in discontinuous dynode geometry has to be utilized. The number of allowed MCPs for it can be determined by the Child Langmuir law. It is a relationship between current density and applied voltage across a vacuum gap of a cathode and anode 
in vacuum. From the knowledge of these parameters the number of microchannel plates that can be used in the discontinuous dynode geometry can be determined.

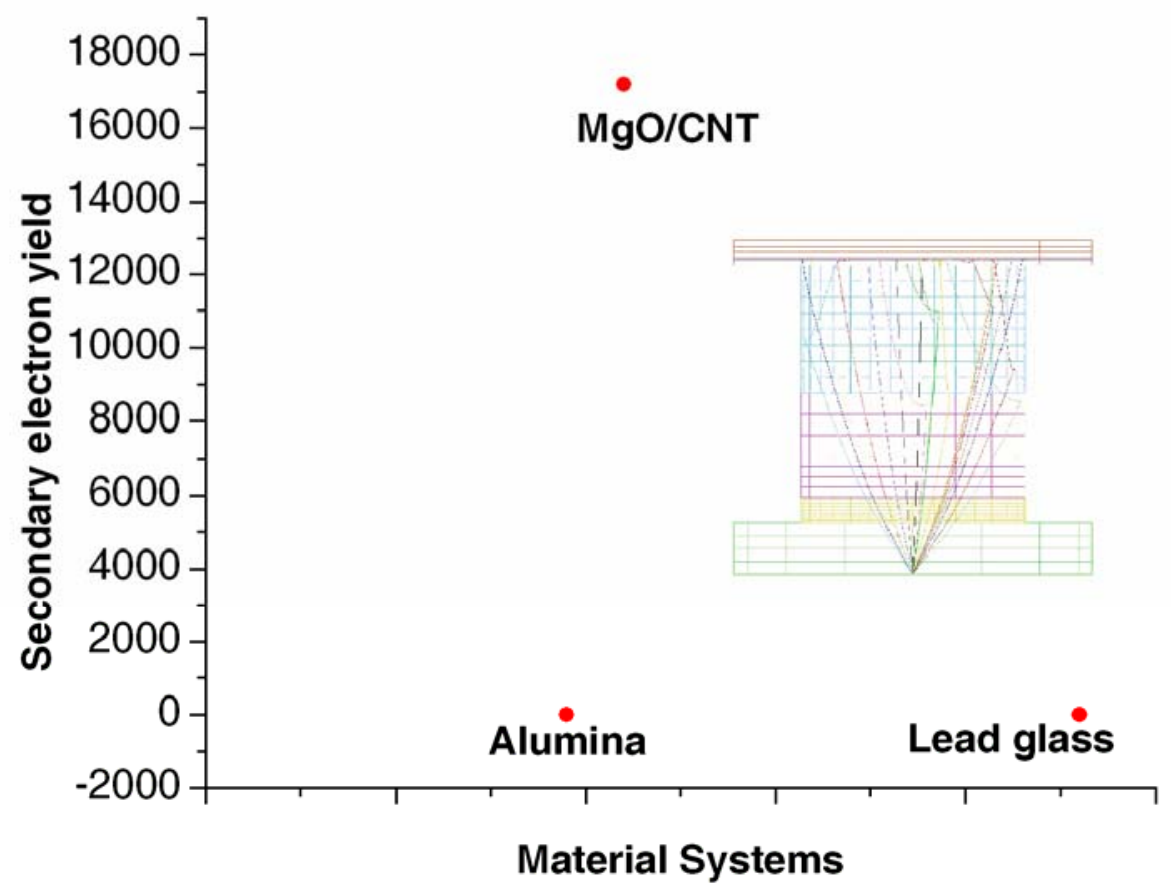

Figure 4.23. Simulation results various materials systems considering them as the emissive layer along the channel that had the similar dimensions as the fabricated MCP in the present work. Among the material systems MgO-coated CNTs show the highest secondary yield. This shows that high multiplication achieved for the relatively small aspect ratio channel was a result of $\mathrm{MgO}-\mathrm{CNT}$. 


\section{CONCLUSION}

The main focus of this research work was to develop efficient cold electron sources based on carbon nanotubes and nanowires and enhance their emission current utilizing an electron multiplier microchannel plate (MCP). Nanomaterials are highly promising for field emitter application, because of their high aspect ratio $(\sim 1000)$ and atomically sharp apex, which enhances local field and lowers threshold field for electron emission ( $\sim 0.5-1$ $\mathrm{V} / \mu \mathrm{m})$. The novel emitter consisted of thin-MWNTs, multistage carbon nanotubes i.e. thin-MWNTs/SWNTs grown on MWNTs and multistage tungsten oxide nanowires. Theoretical analysis was also performed to validate the multistage effect on achieving efficient field emission. This research work also demonstrated the design and fabrication of a novel MCP able to amplify field emission current in the range of few mA-A. The design and material system for the MCP was based on charged particle optics (CPO) simulation program. The optimum MCP channel aspect ratio and tilt were determined from the simulation. Finally the emission current amplification from aligned carbon nanotubes field emitters grown on porous silicon and the novel MCP were demonstrated. The key achievements of our investigations are listed below.

\subsection{MICRO CHANNEL PLATE SIMULATION}

- Optimum aspect ratio for achieving higher gain from MCP was determined to be as 40 from the simulation of a straight/non-tilted channel.

- Optimum tilt angle of the channel for further improving gain of the non-tilted channel was determined to be in the range of $5-10^{\circ}$. 


\subsection{SECONDARY EMISSION CHARACTERIZATION}

- Secondary emission of $\mathrm{MgO}$ synthesized by various methods was determined from the secondary emission characterization experiments.

- $\mathrm{MgO}$ coatings obtained from powder suspension were determined to have higher secondary emission $(\sim 18-25)$ compared to that obtained from sol-gel $(\sim 2-4)$. This might be attributed to the structural crystal structure of the $\mathrm{MgO}$ obtained from suspensions which was more porous and had larger crystal size. Thus leading to lesser scattering and minimizing the losses of generated secondary electrons.

\subsection{SYNTHESIS OF EFFICIENT FIELD EMITTERS}

\subsection{1 thin-MWNTs}

- High density thin-MWNTs were synthesized by thermal chemical vapor deposition.

- Density of thin-MWNTs was increased by decreasing the amount of $\mathrm{Al}_{2} \mathrm{O}_{3}$ in the catalyst solution. The high density of nanotubes led to their vertical orientation.

- The thin-MWNTs showed low turn-on electric field $(\sim 0.5-1 \mathrm{~V} / \mu \mathrm{m})$ and high emission current $(\sim 2.5 \mathrm{~mA})$ which could be attributed to their wall thinness and high aspect ratio $(\sim 1500)$ resulting in high field enhancement factor $(\sim 9300)$.

- Emission current was stable with an average fluctuation of $2 \%$ under DC bias. 


\subsubsection{MULTISTAGE CNTs}

- Synthesized and analyzed field emission properties of carbon nanotube multistage emitter arrays grown on porous silicon by catalytic thermal chemical vapor deposition.

- The vertically oriented multistage array structures consisted of SWNT and thinMWNT grown on MWNT, confirmed by TEM and Raman analysis.

- Higher field emission current $\sim 32$ times $(\sim 450 \mu \mathrm{A})$ and low threshold field $\sim 1.5$ times $(0.4 \mathrm{~V} / \mu \mathrm{m})$ were obtained for these structures in comparison to only MWNTs (I:14 $\mu \mathrm{A}$, turn-on field $0.6 \mathrm{~V} / \mu \mathrm{m})$.

- The enhanced field emission results for these multistage emitters are a consequence of higher field concentration, which is $\sim 3$ times more than MWNTs.

\subsubsection{MULTISTAGE TUNGSTEN OXIDE NANOWIRES}

- Tungsten oxide $\left(\mathrm{W}_{18} \mathrm{O}_{49}\right)$ nanowires were grown by thermal chemical vapor deposition in a mixture of $\mathrm{CH}_{4}$ and $\mathrm{H}_{2}$ on an electrochemically sharpened tungsten tip.

- The field emission measurements showed a low threshold field of $\sim 0.95 \mathrm{~V} / \mu \mathrm{m}$ which is $\sim 2$ times lower than previously reported. High emission current of $\sim 170 \mu \mathrm{A}$ per single emitter was also achieved. The improved emission properties are a consequence of large field enhancement factor of $\sim 19800$.

- High emission current of a few $\mu \mathrm{A}$ was also observed in relatively poor vacuum at $3 \times 10^{-3}$ Torr, and the emission properties were recoverable at $1 \times 10^{-6}$ Torr. 


\section{4 FABRICATION OF NOVEL HIGH SECONDARY EMISSION MCP}

- Utilized the extremely high secondary emission properties of magnesium oxide coated carbon nanotubes (CNT) for the fabrication of a novel microchannel plate.

- The MCP was characterized for electron multiplication by placing it between a nanotube emitter array cathode and an anode.

- In addition, to enhancing the field emission by $\sim 25$ times (from $40 \mu \mathrm{A}$ with out MCP to $1.1 \mathrm{~mA}$ with MCP), the MCP was also observed to protect the nanotubes from irreversible damage during vacuum arcing.

- The obtained results are a significant technological advance and this research holds promise for electron source in new generation lightweight, efficient and compact microwave devices for telecommunications in satellites or space applications. 


\section{RECOMMENDATIONS FOR FUTURE WORK}

In the current work, new and efficient field emitters have been fabricated and a method to enhance the emission current from field emitters through an electron multiplier channel plate has been demonstrated. Improvisation of the present work are listed below.

\section{TUNGSTEN OXIDE NANOWIRE MULTISTAGE FIELD EMITTERS}

- Fabricate an electrochemically sharpened tungsten tip with a narrower diameter i.e. in the range of few nm to further improve field enhancement thus improving field emission properties.

- Design a control circuit that can control applied voltage thus achieving higher emission stability.

\section{CARBON NANOTUBE MULTISTAGE FIELD EMITTERS}

- Improve the density of CNT towers in the cathode utilizing lithographic techniques.

- Growth of aligned thin-MWNTs/SWNTs on top of the MWNT to improve the field enhancement factor thus improving emission properties.

\section{MICROCHANNEL PLATE}

- Fabricate high aspect ratio channels utilizing mechanical punch.

- Study various coating methods such as atomic layer deposition for CNT catalyst and $\mathrm{MgO}$ along MCP channels.

- Study amplification with various MgO coating thickness on CNTs.

- Characterize MCP with various tilt angles using thermionic gun and manipulator.

- Test MCP in discontinuous dynode form. 


\section{REFERENCES}

1. Choi, W. B; Chung, D. S; Kang, J. H; Kim, H. Y; Jin, J. Y; Han. I. T; Lee, Y. H; Jung, J. E; Lee, N. S; Park, G. S; Kim, J. M. (1999) Appl. Phys. Lett., 75, 3129.

2. Zhang, J., Yang, G., Lee, Y. Z., Chang, S., Lu, J. P., and Zhoi, O. (2006), Appl. Phys. Lett. 89064106.

3. Saito, Y. \& Uemura, S. (2000) Carbon 38, 169.

4. Teo, K. B. K; Minoux, E; Hudanski, L; Peauger, F; Schnell, J. P; Gangloff, L; Legagneux, P; Dieumegard, D; Amartunga, G. A. J; Milne, A. I. (2005) Nature, 437, 968 .

5. Seelaboyina, R., Huang, J., and Choi, W. B. (2006), Appl. Phys. Lett., 88194104.

6. Wiza, J. L. (1979), Nuclear Instruments and Methods, 162587.

7. Eschard, E.; Manley, B. W. (1971) Acta Electronica,14, 19.

8. Yi, W.; Jeong, T.; Jin, S.; Yu, S. G.; Lee, J.; Kim, J. M (2000) Review of Scientific Instruments, 71, 4165.

9. Yi, W.; Jin, S.; Jeong, T.; Lee, J.; Yu, S. G.; Choi, Y.; Kim, J. M. (2000), Appl. Phys. Lett., 771716.

10. Khazan, A. D. Transducers and Their Elements: Design and Application, Prentice Hall: New York, 1993.

11. Loty, C. Acta Electronica 1971, 14, 107-119.

12. Choi, Y. S., and Kim, J. M. (2000), IEEE Transactions on Electron Devices, 47 1293.

13. Yi, W; Yu, S. G.; Lee, W; Han, I, T; Jeong, T; Woo, Y; Lee, J; Jin, S; Choi, W. B; Heo, J; Jeon, D; Kim, J. M. (2001) Journal of Appl. Phys. 89, 4091.

14. Kim. W. S; Yi, W.; Jin, S.; Jeong, T.; Lee, J.; Yu, S. G.; Choi, Y.; Kim, J. M; Jeong, H, J; Shin, Y. M; Lee, Y. H.(2002) Appl. Phys. Lett., 81, 1098.

15. Heo, J. N; Lee, J. H; Jeong, T. W; Lee, C. S; Kim, W. S; Jin, Y. W; Kim, J. M; Yu, S. G; Yi, W. K; Park, S. H; Oh, T. S; Yoo, J. B. (2005) Appl. Phys. Lett., 87, 114105-1.

16. Cazaux, J. (1999), J. Appl. Phys., 851137. 
17. Deledik, K., Emelianchik, I. (2003), Nuclear Phys, 125394.

18. Govyadinov, A., Emelianchik I. (1998), Nuclear Instruments and Methods in Physics Research A, 41667.

19. Bonard, J. M., Salvetat, J. P., Sto"ckli, T., de Heer, W. A., Forro', L., and Cha^ telain, A. (1998), Appl. Phys. Lett. 73918.

20. Dean, K. A., and Chalamala, B. R. (1999), Appl. Phys. Lett. 753017.

21. Wadhawan, A., Stallcup R. E., Stephens, K. F., Perez, J. M. and Akwani, I. A.(2001), Appl. Phys. Lett. 791867.

22. Kim, D. H., Jang, H. K., Lee, S. Y., and Lee, H. R. (2004), Nanotechnology 15 1433.

23. Tondare, V. N., Druten, N. J., Hagen, C. W., and Kruit, P. (2003), J. Vac. Sci. Technol. B. 211602.

24. Zhirnov V V, Wojak G J, Choi W B, Cuomo J J and Hren J J (1997) J. Vac. Sci. Technol. A 151733.

25. Qiu, H., Lu, Y. F., and Mai, Z. H. (2002), J. Appl. Phys. 91440.

26. Solis, J. L., Saukko, S., Kish, L., Granqvist, C. G., and Lantto, V. (2001), Thin Solid Films 391255.

27. Vida, V. K., Josepovits, M., Gyor, and Deák, P. (2003), Microsc. Microanal. 9 337.

28. Gillet, M., Delamare, R. and Gillet, E. (2005), Eur. Phys. J. D 34291.

29. Fumio, O. (1971), J. Appl. Phys 42256.

30. Umnov, A.G., Shiratori, Y., Hiraoka, H. (2003) Appl. Phys. A 77159.

31. Lee, Y. H., Choi, C. H., Jang, Y. H., Kim, E. K., Ju, B. K., Min, N. K., Ahn, J. H. (2002), Appl. Phys. Lett. 81745.

32. Li, Y., Bando, Y., and Goldberg, D. (2003), Adv. Mater 151294.

33. Zhou, J., Li G., Zhi, S. D., Chen, J., She, J. C., Xu, N. S., Yang, R., and Wang, Z. L. (2005), Appl. Phys. Lett. 87223108.

34. Liu, J., Zhang, Z., Zhao, Y., Su, X., Liu, and Wang. (2005), Small 3310. 
35. Modinos, A., Field, Thermionic and Secondary Electron Emission Spectroscopy (1984), Plenum Press, New York.

36. Gomer, R., Field emission and field ionization (1961), Harvard university press, MA.

37. Fursey, G., Field emission in vacuum microelectronics (2003), Kluwer academic/ Plenum publishers, New York.

38. Oostrom, V. A. G. J. (1965), Validity of Fowler-Nordheim model for field electron emission, Thesis, University of Amsterdam.

39. Fowler, R. H. and Nordheim, L. W. (1928), Proc. R. Soc. London. Ser. A 119, 173.

40. Iijima, S. (1991), Nature 35456.

41. Saito, R., Dresselhaus, G., and Dresselhaus, M. S. (1998), Physical properties of carbon nanotubes, World scientific.

42. Rinzler, A. G., Hafner, J. H., Nikolaev, P., Lou, L., Kim, S. G., Tomanek, D., Nordlander, P., Colbert, D. T., and Smalley, R E. (1995), Science, 2691550.

43. Bonard, J. M., Salvetat, J. P., Stockli, T., Forro, L., and Châtelain, A. (1999), Appl. Phys. A., 69245.

44. Jong, N. D., Amaratunga, A. J., Teo, K. B. K. and Milne, W. I. (2005), Appl. Phys. Lett., 87133118.

45. Minoux, E., Groening, O., Teo, K. B. K., Dalal, H. S., Gangloff, L., Schnell P. J., Hudanski, L., Bu, Y. Y., Vincent, P., Legeneux, P., Amartunga J. A. G., Milne, W. I. (2005), Nanoletters, 52135.

46. Cheng, Y., and Zhou, O. (2003), C. R. Physique, 41021.

47. Choi, W. B., Yun, M. J., Jin, Y. W. (2005), US20056976897.

48. Fan, S. S., Chapline, M. G., Franklin, N. R., Tombler, T. W., Cassell, A. M. and Dai, H. (1999), Science, 283512.

49. Dai, H., Fan, S.S., Chapline, A., Franklin, M. P., Thomas, T. S. (2001), US20016232706.

50. Choi, W. B. (2002), US20026440761. 
51. Wei, Y., Xie, C., Dean, K. A. and Coll F. B. (2001), Appl. Phys. Lett., 794527.

52. Nilsson, L., Groening, O., Emmenegger, C., Kuettel, o., Schaller, E., Schlapbach, L., Kind, H., Bonard, J. M., and Kern. K. (2000), Appl. Phys. Lett., 762071.

53. Wang, Y. W., Lee, T. C., Lee, C.C. (2005), US2005682112.

54. Lee, C. J. (2003), US20036514113.

55. Zhou, O. Z., Lu, J. (2005), US20056850595.

56. Forbes, R. G., Edgcombe, C. J., and Valdre, U. (2003) Ultramicroscopy 9557.

57. Huang, J. Y., Kempa, K., Jo, S. H., Chen, S., and Ren, Z. F. (2005), Appl. Phys. Lett. 87053110 .

58. Lin, Y., and Joy, D. C. (2005), A new examination of secondary electron yield data, Surf. Interface Anal., 37895.

59. Bruining H., Physics and Applications of Secondary Electron Emission (1954), Pergamon Press: London.

60. Joy, D. C., UTK Metrology Group http://web.utk.edu/ srcutk/htm/interact.htm.

61. Dionne, G. F. (1975), J. Appl. Phys. 463347.

62. Nickles, N. E.(2002), The role of bandgap in the secondary electron emission in small bandgap semiconductors: studies of graphitic carbon, Ph.D Dissertation, Utah state university.

63. Thomson, C.D. (2004), Measurements of the secondary electron emission properties of insulators, Ph.D Dissertation, Utah state university.

64. Ascarelli, P., Cappelli, E., Pinzari, F., Rossi, M. C., Salvatori. S., Merli, P. G. and Migliori, A. (2000), J. Appl. Phys., 89689.

65. Whetten, N. R., and Laponsky, A. B. (1959), J. Appl. Phys., 30432.

66. Cazaux, J., Kim, K. H., Jbara, O., and Salace, G. (1991), J. Appl. Phys. 70960.

67. Seiler, H. J. (1983), J. Appl. Phys. 54 R1.

68. Salow, H. (1940), Z. techn. Phys., 41434.

69. Dekker, A. J., Solid State Phys. (1957), Prentice-Hall: NJ. 
70. Alig, R. C. and Bloom, S. (1978), J. Appl. Phys., 493476.

71. Kanaya, K., Ono, S., and Ishigaki, K. (1978), J. Phys. D: Appl. Phys., 112425.

72. Khairi, L. and Bastawros, A. M. (1981), J. Appl. Phys., 535239.

73. Cazaux, J. (2003), Thin Solid Films, 434303.

74. Adams, J., and Manley, B. W. (1966), IEEE Transactions on Nuclear Science, 13 88.

75. Lee, C.J., Kim, D.W., Lee, T.J., Choi, Y.C., Park, Y.S., Kim, W.S., Lee, Y.H., Choi, W. B., Lee, N. S., Kim, J. M., Choi, Y. G., and Yu, S.C. (1999), Appl. Phys. Lett. 75, 1721.

76. Kerfriden, S., Nahle, A. H., Campbell, A. S., Walsh, F.C., and Smith, J. R. (1998), Electrochimica Acta 43193.

77. Klinke, C., Hannon, J. B., Gignac, L., Reuter, K., and Avouris, P.(2005), Phy. Chem. B. Lett. 10911787.

78. Uri Cohen, Gene Patrick Bonnie, (2003) US2003 6635184.

79. Raj N. Singh, (1983) US19834381216.

80. Kalman F. Zsamboky, (1991) US19915058799.

81. Journal of Applied Physics, 922855.

82. Journal of Solid State Chemistry.

83. Applied Surface Science, 17462.

84. Therese, G. H., and Kamath, P. V. (2000), Chem. Mater., 121195.

85. Sarkar, P. and Nicholson, S. P. (1996), J. Am. Ceram. Soc, 791987.

86. Ferrari, B.,Moreno, R., Sarkar, and P., Nicholson, P.S., (2000), Journal of the European Ceramic Society, 2099.

87. Riccardis, M. F., Carbone, D., and Rizzo, A, (2007), Journal of Colloid and Interface Science, 307109.

88. Users gide for CPO2D and CPO3D (2004). 
89. Raspereza, A. V. (1997), Applied Surface Science 111, 295.

90. Lee, J., and Jeong, T., (2001) Applied Surface Science, 174, 62.

91. Heo, J. N, Kim, W. S, Jeong, T. W, Yu, S. G, Lee, J. H, Lee, C. S, Yi, W. K, Yoo, J. B and Kim, J. M (2002), Physica B, 323, 174.

92. Yu, S. G., Yi, W., Jeong, T., Kim, W. S., Lee, J., Heo, J., Lee, C. S., Yoo, J. B., Lee, H. Y., and Kim, J. M (2002), Physica B, 323, 177.

93. Jacobs, H., Freely, J., and brand, A. F. (1952), Phys. Rev., 84, 877.

94. Wenzhi Li, Hao Zhang, Chaoying Wang, Yun Zhang, Liwen Xu, Ke Zhu, and Sishen Xie, Appl. Phys. Let. 70, 2684 (1997).

95. Young Chul Choi, Y. M. Shin, D. J. Bae, S. C. Lim, Y. H. Lee and B. S. Lee, (2001), Diamond. Relate. Mater. 10, 1457.

96. S. Bandow, S. Asaka, Y. Saito, A.M. Rao, L. Grigorian, E. Richter, and P.C. Eklund, (1998) Phys. Rev. Lett. 80, 3779.

97. W.A. de Heer, J.-M. Bonard, K. Fauth, A. Chatelain, L. Forro, and D. Ugate, (1997) Adv. Mater. 9, 87.

98. J. M. Bonard, F. Maier, T. Stockli, A. Chatelain, W.A. de Heer, J.-P. Salvetat, and L.Forro, (1998) Ultramicroscopy 73, 7.

99. F.H. Kaatza, M.P. Siegalb, D.L. Overmyerb, P.P. Provenciob, and J.L. Jackson, (2003) Mater. Sci. \& Eng. C 23, 41.

100. Y.C. Choi, Y.M. Shin, S.C. Lim, D.J. Bae, Y.H. Lee, B.S. Lee, and D.-C. Chung, (2000) J. Appl. Phys. 88, 4898.

101. Frey, G. L., Rothschild A, Sloan J, Rosentsveig R, Popovitz-Biro R and Tenne R (2001) J. Solid State Chem., 162300.

102. Smith R C, Forrest R D, Carey J D, HsuWK and Silva S R P (2005) Appl. Phys. Lett., 87013111.

103. Lehmanna V and Rönnebeck, S (1999), Journal of The Electrochemical Society, 146, 2968.

104. Dresselhausas M S, Dresselhaus G, Saito R, and Jorio A (2005), Physics Reports, 40947. 
105. Seelaboyina R., Huang J, Park J, Kang D H, and Choi W B (2006), Nanotechnology 17, 4840.

106. Chang C S, Chattopadhyay S, Chen L C, Chen K H, Chen C W, Chen Y F, Collazo R, and Sitar Z (2003), Physical Review B 68, 125322. 


\section{APPENDIX 1}

\section{LAMINATION AND SINTERING PROCEDURE}

The lamination procedure for $\mathrm{Al}_{2} \mathrm{O}_{3}$ green tape is as follows,

1. Preheat the platens to a temperature of $80-90^{\circ} \mathrm{C}$ for $5-10$ minutes.

2. Stack desired number of tape layers from A side to B side with each layer perpendicular.

3. Place the above sample between two layers of mylar.

4. Cover the mylar with aluminum on top and bottom.

5. Place it in the press and use 1000-2000 psi of pressure.

6. Hold the pressure for 5 minutes.

7. Release the pressure and let it stand for a few minutes to cool.

8. Remove the laminated sample.

Note: Temperature and pressures should be adjusted depending on number of layers and size of the green tape to be laminated.

Following is the sintering cycle for $\mathrm{Al}_{2} \mathrm{O}_{3}$ green tape.

1. Room temperature to $500^{\circ} \mathrm{C}$ at a rate of $3^{\circ} \mathrm{C}$ /minute.

2. $500{ }^{\circ} \mathrm{C}-625^{\circ} \mathrm{C}$ at a rate of $1{ }^{\circ} \mathrm{C} /$ minute.

3. $625^{\circ} \mathrm{C}-1500^{\circ} \mathrm{C}$ at a rate of $5^{\circ} \mathrm{C} /$ minute.

4. $1500{ }^{\circ} \mathrm{C}-1550{ }^{\circ} \mathrm{C}$ at a rate of $1{ }^{\circ} \mathrm{C} /$ minute or desired sintering temperature.

5. Hold for 3 hours at $1550^{\circ} \mathrm{C}$.

6. $1550^{\circ} \mathrm{C}$ to room temperature furnace cooling. 
Note: Samples i.e. green tape should be sintered in air atmosphere, the samples should be placed between two porous $\mathrm{Al}_{2} \mathrm{O}_{3}$ cover plates with at least $0.77 \mathrm{gm} / \mathrm{cm}^{2}$ to maintain flatness. 


\section{APPENDIX 2}

\section{COPPER (CU) ELECTRO PLATING}

\section{CLEANING PROCEDURE}

1. Solvent decrease using isopropyl alcohol - Removes oil and grease; followed by vigorous water rinse.

2. Alkali Soak with $\mathrm{NaOH}$ for $5 \mathrm{Min}-\mathrm{Hot} 150^{\circ}$ to $180^{\circ} \mathrm{F}$ to further clean $\mathrm{Cu}$ surface, condition laminate, improve surface wetting followed by warm water rinse.

3. Persulfate Etch for 2 min - Produces matte pink copper surface: ammonium persulfate - $200 \mathrm{gm} / \mathrm{L}$; sulfuric acid - $10 \mathrm{ml} / \mathrm{L}$. Followed by water rinse.

4. $10 \%$ Sulfuric Immersion for 2 min - Removes insoluble salts (Step \#3). Followed by water rinse.

5. $33 \%$ HCL Immersion for $2 \mathrm{~min}-$ To protect from harmful drag-in.

Note: For $\mathrm{Al}_{2} \mathrm{O}_{3}$ most of the times the above mentioned procedure does not yield good results, so instead of the above cleaning solutions immerse sintered alumina in molten $\mathrm{NaOH}$ (heat $\mathrm{NaOH}$ to $425^{\circ} \mathrm{C}$ ) for $15 \mathrm{~min}$. Before immersing the alumina templates should also be heated till $425^{\circ} \mathrm{C}$, otherwise they may break due to thermal shock. Please refer patents 70-72 for other etching solutions and conditions.

\section{COPPER DEPOSITION}

After the above cleaning procedure follow the procedure explained below to deposit

$\mathrm{Cu}$, immerse the $\mathrm{Al}_{2} \mathrm{O}_{3}$ templates in the solutions for the specified time

1. Sensitizer Solution C for 2 min. - Seeds epoxy/glass laminate. Followed by water rinse. 
2. Activator Solution D for 2 min. immersion - Activates an autocatalytic layer for copper deposition. Followed by thorough water rinse.

3. 5\% Sulfuric dip for 3-5 min. - Speeds electroless copper deposition, protects from drag-in contamination. Followed by water rinse.

4. Electroless Copper Parts A and B for 5-10 min. - Solution were prepared by mixing $\mathrm{A}$ and $\mathrm{B}$ in equal volume and it was operated at $30-45^{\circ} \mathrm{C}$ with mild agitation. Followed by water rinse. 


\section{APPENDIX 3}

\section{CHARGED PARTICLE OPTICS (CPO) CODE FOR STRAIGHT CHANNEL SIMULATION}

CPO3DS, 81st example file, channel electron multiplier temp81.dat name of hidden output file, for processed data temp81a.dat name of main output data file, for ray data $\mathrm{p} \quad \mathrm{n} / \mathrm{p} / \mathrm{m} / \mathrm{a}$ for segment printing level

1100 voltage reflection symmetries in $\mathrm{x}, \mathrm{y}, \mathrm{z}, \mathrm{x}=\mathrm{y}$ planes 2 number of different voltages, time dependence

0.00110 allowed consistency error, side/length ratio check $\mathrm{n}$ apply inscribing correction $(\mathrm{a} / \mathrm{s} / \mathrm{n}=$ always/sometimes/never) edi evenly-divided disc $\quad * * *$ Source disc $0.07500-0.1 \quad$ radius, centre of disc 000 any other point on axis

11 numbers of 2 applied voltages (can be same)

20 number of subdivisions

cylindrical electrode $\quad * * *$ shield

$0.07500-0.1$ radius, centre of 1 st end

000 centre of 2 nd end

11 numbers of 2 applied voltages (can be same)

44 total nr of subdivs and 0 , or subdivs along and around axis disc, uneven radial division $* * *$ multiplier face

$0.075000 \quad 1$ st radius, centre of disc 
0.050012 nd radius, any other point on axis

11 numbers of 2 applied voltages (can be same)

24 total $\mathrm{nr}$ of subdivs and 0 , or subdivs along radius and around axis

cylindrical electrode $\quad * * *$ source end of channel

0.05000 radius, centre of 1 st end

000.05 centre of 2 nd end

12 numbers of 2 applied voltages (can be same)

05 z's of application points

88 total $\mathrm{nr}$ of subdivs and 0 , or subdivs along and around axis

cylindrical electrode $\quad * * *$ channel, middle part

0.05000 .05 radius, centre of 1 st end

004.95 centre of 2 nd end

12 numbers of 2 applied voltages (can be same)

05 z's of application points

1204 total $\mathrm{nr}$ of subdivs and 0 , or subdivs along and around axis

cylindrical electrode $\quad * * *$ anode end of channel

0.05004 .95 radius, centre of 1 st end

005 centre of 2 nd end

12 numbers of 2 applied voltages (can be same)

05 z's of application points

88 total $\mathrm{nr}$ of subdivs and 0 , or subdivs along and around axis

disc, uneven radial division $* * *$ multiplier face

0.0750051 st radius, centre of disc 
0.05005 .52 nd radius, any other point on axis

22 numbers of 2 applied voltages (can be same)

24 total $\mathrm{nr}$ of subdivs and 0 , or subdivs along radius and around axis

cylindrical electrode $\quad * * *$ shield

0.075005 radius, centre of 1 st end

005.1 centre of 2 nd end

22 numbers of 2 applied voltages (can be same)

44 total $\mathrm{nr}$ of subdivs and 0 , or subdivs along and around axis

edi evenly-divided disc $\quad * * *$ anode

$0.075005 .1 \quad$ radius, centre of disc

004.5 any other point on axis

22 numbers of 2 applied voltages (can be same)

20 number of subdivisions

end of electrode information

$\begin{array}{llll}0 & 0 & 0.5 & \text { final number of segments, number of steps, weight }\end{array}$

1e-07 charge inaccuracy, zero total charge

end of segment information

$0.0000000 \mathrm{E}+00 \quad$ cathode

$1.5000000 \mathrm{E}+03 \quad$ anode

n no more magnetic fields from menu

$\mathrm{n} \mathrm{n} \mathrm{n}$ no more potentials and fields along a line

start of ray information

d direct (d), or mesh (m) method and mesh spacing 
$\mathrm{p}$ 'n/p/m/a' for 'nearlyzero/partial/most/all' printing level, rho/radius

-0.50 .5 minimum and maximum $\mathrm{x}(\mathrm{mm})$ of screen $2 \mathrm{D}$ fields of view

-0.50 .5 minimum and maximum y

-0.25 .2 minimum and maximum $\mathrm{z}$

-0.50 .5 minimum and maximum $\mathrm{x}(\mathrm{mm})$ of rays

-0.50 .5 minimum and maximum y

-0.25 .2 minimum and maximum $\mathrm{z}$

$-1 \mathrm{e}+101 \mathrm{e}+10$ minimum and maximum $\mathrm{vx}(\mathrm{m} / \mathrm{s})$ of rays

$-1 \mathrm{e}+101 \mathrm{e}+10 \quad$ minimum and maximum vy

$-1 \mathrm{e}+101 \mathrm{e}+10$ minimum and maximum $\mathrm{vz}$

-0.50 .5 minimum and maximum rho $\left(=\operatorname{sqrt}\left(x^{* *} 2+y * * 2\right)\right)$

$+\mathrm{s}$ direction of time, secondaries option

rm $1111520-0.5-2504$ seed, min en, kT, add, mult

2e-05 final time (ms)

0.010 .010 initial and max step length, interpolation points

$0.00011 \mathrm{e}-05$ fractional inaccuracies for (1) ray tracing and (2) potentials and fields

1 nr test planes,mult cross, iter foc,phase spc,scatt,quant

0015.09 a,b,c,d of test plane defined by $a^{*} x+b * y+c * z=d$

el 'el' for electron; or 'co' or 'va' for other particles

k kinetic energy $(\mathrm{k})$; or total energy $(\mathrm{t})$ and potential

cylindrical beam

f c 1111 edge disc, random pupil, random re-seeding

$00-0.10$ centre and radius of window 
000 any other point on axis

000.050 .05 centre and radius of pupil

$1201001 \mathrm{e}-06$ n's for source disc and pupil, eV, mA

n calculate space-charges?

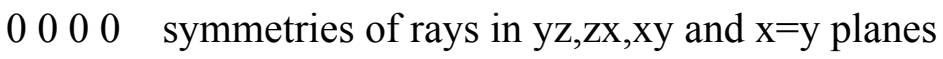

CPO3DS, 81st example file, channel electron multiplier (See xmpl3d82 for tilted channels.)

One channel is simulated. It has diameter $0.1 \mathrm{~mm}$, length $5 \mathrm{~mm}$. The 2 ends are shielded with small field-free boxes. The voltage difference between the ends is 1500V. 20 electrons are injected with energy of $100 \mathrm{eV}$.

When an electron hits the side of the channel it produces secondaries. The energy, direction and current of the primary ray are measured when the primary hits a side and these parameters are used to determine the energy, direction and current of the secondary ray. Each ray represents a set of electrons. In principle there should usually be more than one secondary electron per primary electron, but CPO3DS cannot generate more than one secondary electron, so the currents of the rays carry the information about the number of electrons that are represented by the ray.

3 options are triggered in this example: A Maxwellian distribution of the energies of the secondaries, Lambertian (cosine) distribution of directions, Poisson distribution of the currents. Details of these options are given in Help. The formula for the mean multiplication factor per collision is taken from A. V. Raspereza et al, Submicrochannel plate multipliers, Applied Surface Science 111 (1997) 295301. The formula is 


$$
\text { fmean }=\operatorname{fmax}^{*} \exp (-a m *(1 .-\mathrm{cs})) * 4 \cdot{ }^{*} \mathrm{x} /(1 .+\mathrm{x}) * * 2
$$

where

$$
\begin{aligned}
& \mathrm{x}=\operatorname{sqrt}(\mathrm{cs})^{*} \text { energy/emax, } \\
& \mathrm{cs}=\text { cosine of angle of primary to the normal to the surface, } \\
& \text { energy = primary energy. }
\end{aligned}
$$

According to Raspereza et al (see above):

$$
\text { fmax emax am }
$$

\section{Lead glass $\quad 4.0 \quad 250 \quad 0.5$}

\section{Alumina $\quad 4.8 \quad 350 \quad 0.5$}

They also say that the secondary electrons have a Maxwellian energy distribution, mean value 2.0ev, and a Lambertian (cosine) angular distribution.In the present example, this mean multiplication factor is used for each collision (that is, the 'meanpoisson' option is used, see Help). This represents an approximation to the real process (see Help) but is the only viable choice here.

The 'minimum incident energy' for collisions is given the value $5 \mathrm{eV}$, so if a primary ray has an energy smaller than this the ray is stopped. This is the main cause of rays not reaching the anode. 14 rays reach the anode at the end of the channel. The total of the final currents of all the rays is 6.51 microAmp (see the ray output file). Of this, 6.45 microAmp is due to the successful 14 rays. The initial total current of the 20 rays is 0.0005 microAmp. Therefore the multiplication factor is 12900 in this example. Using 500 rays, the multiplication factor is found to be 62220 .

Run with 500 rays.

Analysed by the program below, giving the output: 
Number of rays $=500$

Number of collisions $=4612$

Total current $=-3.302 \mathrm{E}-02$

Test plane current $=-3.111 \mathrm{E}-02$

Highest current $=-3.499 \mathrm{E}-03$

Program for analysing outputs:

! Program to analyze outputs from xmpl3d81,2, multichannel analysers

! Assumes 'partial' printing level for rays, and that CPO3DS has been used.

$!$

DIMENSION $\mathrm{x}(15)$

CHARACTER phrase*210, current*7, raynumber*10, thetotal*9, \& hitting*8, not_allowed*19

!

! To be set before running $* * * * * * * * * * * * * * * * * * *$

test_plane $=4.75$

OPEN (UNIT=1,FILE='tmp20a.dat',STATUS='OLD')

$! \quad=$ input numbers

! $\quad * * * * * * * * * * * * * * * * * * * * * * * * * * * * * * * * * * * * * * * * * * *$

!

OPEN (UNIT=2,FILE='tempout.dat',STATUS='UNKNOWN')

! $=$ results file

!

current $=$ 'Current' 
raynumber $=$ 'Ray number'

thetotal $=$ 'The total'

hitting $={ }^{\prime}($ Hitting'

not_allowed $=$ 'Secondaries are not'

$\mathrm{n}=0$ ! number of rays

$\mathrm{n} \_\mathrm{c}=0$ ! number of collisions

n_a $=0$ ! number of rays that reach anode

not_all $=0$ ! number of secondary collisions $>290$

highest_current $=0$.

100 CONTINUE

$\operatorname{READ}\left(1,{ }^{\prime}(\mathrm{A} 210)^{\prime}\right)$ phrase

IF (phrase(1:8).EQ.hitting) THEN

! Read the currents for the rays that have reached the test plane test_current $=0$.

DO $\mathrm{i}=1, \mathrm{n}$

$\operatorname{READ}(1, *)(x(j), j=1,15)$

IF (abs(x(10) - test_plane).LT.0.01) THEN

! This ray has reached the test plane

$\mathrm{n} \__{-} \mathrm{a}=\mathrm{n} \_\mathrm{a}+1$

test_current $=$ test_current $+\mathrm{x}(15)$

highest_current $=\min ($ highest_current, $\mathrm{x}(15))$

ENDIF

ENDDO 
WRITE $\left(2,{ }^{\prime}(" N u m b e r\right.$ of rays = ",I4)') n

WRITE (2,'("Number reaching anode = ",I4)') n_a

WRITE $\left(2,{ }^{\prime}(\right.$

\& "Number stopped because secondaries are not allowed"/

\& "to be produced more than 29 times =",I4)') not_all

WRITE (2,'("Total number of collisions = ",I4)') n_c

WRITE $(2$, ,("Total of final ray currents = ",1PE10.3)')

\& total_current

WRITE $(2$, ,'("Total current at test plane = ",1PE10.3)')

\& test_current

WRITE (2,'("Highest single current = ",1PE10.3)')

\& highest_current

STOP

ELSEIF (phrase(1:10).EQ.raynumber) THEN

$\mathrm{n}=\mathrm{n}+1$

ELSEIF (phrase(1:7).EQ.current) THEN

$\mathrm{n}_{-} \mathrm{c}=\mathrm{n}_{-} \mathrm{c}+1$

ELSEIF (phrase(1:19).EQ.not_allowed) THEN

not_all $=$ not_all +1

! Skip next 3 lines:

READ (1,'(A210)') phrase

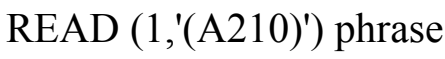

READ (1,'(A210)') phrase 
ELSEIF (phrase(1:9).EQ.thetotal) THEN

$\operatorname{READ}\left(1,{ }^{*}\right)$ total_current

ENDIF

GOTO 100

STOP

END

Output file for a non-tilted channel with aspect ratio 40 channel

\begin{tabular}{|c|c|c|c|c|c|c|}
\hline \multicolumn{7}{|c|}{ Intial values } \\
\hline $\mathrm{x}(\mathrm{mm})$ & $\mathrm{y}(\mathrm{mm})$ & $\mathrm{z}(\mathrm{mm})$ & $\mathrm{v}_{\mathrm{x}}(\mathrm{m} / \mathrm{sec})$ & $\mathrm{v}_{\mathrm{y}}(\mathrm{m} / \mathrm{sec})$ & $\mathrm{v}_{\mathrm{z}}(\mathrm{m} / \mathrm{sec})$ & en \\
\hline $0.00 \mathrm{E}+00$ & $0.00 \mathrm{E}+00$ & $-1.00 \mathrm{E}-01$ & $-1.38 \mathrm{E}+06$ & $9.61 \mathrm{E}+04$ & $5.77 \mathrm{E}+06$ & $1.00 \mathrm{E}+02$ \\
\hline $0.00 \mathrm{E}+00$ & $0.00 \mathrm{E}+00$ & $-1.00 \mathrm{E}-01$ & $-1.51 \mathrm{E}+06$ & $-2.96 \mathrm{E}+05$ & $5.73 \mathrm{E}+06$ & $1.00 \mathrm{E}+02$ \\
\hline $0.00 \mathrm{E}+00$ & $0.00 \mathrm{E}+00$ & $-1.00 \mathrm{E}-01$ & $-1.43 \mathrm{E}+06$ & $1.77 \mathrm{E}+05$ & $5.75 \mathrm{E}+06$ & $1.00 \mathrm{E}+02$ \\
\hline $0.00 \mathrm{E}+00$ & $0.00 \mathrm{E}+00$ & $-1.00 \mathrm{E}-01$ & $1.02 \mathrm{E}+06$ & $1.52 \mathrm{E}+06$ & $5.64 \mathrm{E}+06$ & $1.00 \mathrm{E}+02$ \\
\hline $0.00 \mathrm{E}+00$ & $0.00 \mathrm{E}+00$ & $-1.00 \mathrm{E}-01$ & $-8.41 \mathrm{E}+05$ & $-9.05 \mathrm{E}+05$ & $5.80 \mathrm{E}+06$ & $1.00 \mathrm{E}+02$ \\
\hline $0.00 \mathrm{E}+00$ & $0.00 \mathrm{E}+00$ & $-1.00 \mathrm{E}-01$ & $4.54 \mathrm{E}+05$ & $5.87 \mathrm{E}+05$ & $5.88 \mathrm{E}+06$ & $1.00 \mathrm{E}+02$ \\
\hline $0.00 \mathrm{E}+00$ & $0.00 \mathrm{E}+00$ & $-1.00 \mathrm{E}-01$ & $1.78 \mathrm{E}+06$ & $1.87 \mathrm{E}+05$ & $5.65 \mathrm{E}+06$ & $1.00 \mathrm{E}+02$ \\
\hline $0.00 \mathrm{E}+00$ & $0.00 \mathrm{E}+00$ & $-1.00 \mathrm{E}-01$ & $4.98 \mathrm{E}+05$ & $3.74 \mathrm{E}+05$ & $5.90 \mathrm{E}+06$ & $1.00 \mathrm{E}+02$ \\
\hline $0.00 \mathrm{E}+00$ & $0.00 \mathrm{E}+00$ & $-1.00 \mathrm{E}-01$ & $-1.42 \mathrm{E}+06$ & $-1.06 \mathrm{E}+06$ & $5.66 \mathrm{E}+06$ & $1.00 \mathrm{E}+02$ \\
\hline $0.00 \mathrm{E}+00$ & $0.00 \mathrm{E}+00$ & $-1.00 \mathrm{E}-01$ & $1.13 \mathrm{E}+06$ & $-2.59 \mathrm{E}+05$ & $5.82 \mathrm{E}+06$ & $1.00 \mathrm{E}+02$ \\
\hline $0.00 \mathrm{E}+00$ & $0.00 \mathrm{E}+00$ & $-1.00 \mathrm{E}-01$ & $-8.71 \mathrm{E}+05$ & $1.20 \mathrm{E}+05$ & $5.86 \mathrm{E}+06$ & $1.00 \mathrm{E}+02$ \\
\hline $0.00 \mathrm{E}+00$ & $0.00 \mathrm{E}+00$ & $-1.00 \mathrm{E}-01$ & $-1.27 \mathrm{E}+06$ & $-1.03 \mathrm{E}+06$ & $5.70 \mathrm{E}+06$ & $1.00 \mathrm{E}+02$ \\
\hline $0.00 \mathrm{E}+00$ & $0.00 \mathrm{E}+00$ & $-1.00 \mathrm{E}-01$ & $9.39 \mathrm{E}+05$ & $1.40 \mathrm{E}+06$ & $5.69 \mathrm{E}+06$ & $1.00 \mathrm{E}+02$ \\
\hline $0.00 \mathrm{E}+00$ & $0.00 \mathrm{E}+00$ & $-1.00 \mathrm{E}-01$ & $-5.53 \mathrm{E}+05$ & $-1.15 \mathrm{E}+06$ & $5.79 \mathrm{E}+06$ & $1.00 \mathrm{E}+02$ \\
\hline $0.00 \mathrm{E}+00$ & $0.00 \mathrm{E}+00$ & $-1.00 \mathrm{E}-01$ & $-1.55 \mathrm{E}+06$ & $4.32 \mathrm{E}+05$ & $5.71 \mathrm{E}+06$ & $1.00 \mathrm{E}+02$ \\
\hline $0.00 \mathrm{E}+00$ & $0.00 \mathrm{E}+00$ & $-1.00 \mathrm{E}-01$ & $2.57 \mathrm{E}+05$ & $1.39 \mathrm{E}+06$ & $5.76 \mathrm{E}+06$ & $1.00 \mathrm{E}+02$ \\
\hline $0.00 \mathrm{E}+00$ & $0.00 \mathrm{E}+00$ & $-1.00 \mathrm{E}-01$ & $3.40 \mathrm{E}+05$ & $1.83 \mathrm{E}+05$ & $5.92 \mathrm{E}+06$ & $1.00 \mathrm{E}+02$ \\
\hline $0.00 \mathrm{E}+00$ & $0.00 \mathrm{E}+00$ & $-1.00 \mathrm{E}-01$ & $4.63 \mathrm{E}+05$ & $-1.36 \mathrm{E}+06$ & $5.75 \mathrm{E}+06$ & $1.00 \mathrm{E}+02$ \\
\hline
\end{tabular}




\begin{tabular}{|c|c|c|c|c|c|c|}
\hline $0.00 \mathrm{E}+00$ & $0.00 \mathrm{E}+00$ & $-1.00 \mathrm{E}-01$ & $3.18 \mathrm{E}+05$ & $-1.13 \mathrm{E}+06$ & $5.81 \mathrm{E}+06$ & $1.00 \mathrm{E}+02$ \\
\hline $0.00 \mathrm{E}+00$ & $0.00 \mathrm{E}+00$ & $-1.00 \mathrm{E}-01$ & $-7.71 \mathrm{E}+05$ & $2.19 \mathrm{E}+05$ & $5.88 \mathrm{E}+06$ & $1.00 \mathrm{E}+02$ \\
\hline
\end{tabular}

\begin{tabular}{|c|c|c|c|c|c|c|c|}
\hline \multicolumn{8}{|c|}{ Final values } \\
\hline $\mathrm{x}(\mathrm{mm})$ & $\mathrm{y}(\mathrm{mm})$ & $\mathrm{z}(\mathrm{mm})$ & $\mathrm{v}_{\mathrm{x}}(\mathrm{m} / \mathrm{sec})$ & $\mathrm{v}_{\mathrm{y}}(\mathrm{m} / \mathrm{sec})$ & $\mathrm{v}_{\mathrm{z}}(\mathrm{m} / \mathrm{sec})$ & $\mathrm{t}(\mathrm{sec})$ & $\mathrm{I}(\mathrm{mA})$ \\
\hline $2.79 \mathrm{E}-02$ & $-1.83 \mathrm{E}-02$ & $5.09 \mathrm{E}+00$ & $5.33 \mathrm{E}+05$ & $1.25 \mathrm{E}+05$ & $5.15 \mathrm{E}+06$ & $1.34 \mathrm{E}-06$ & $-1.35 \mathrm{E}-04$ \\
\hline $3.59 \mathrm{E}-02$ & $-3.46 \mathrm{E}-02$ & $4.43 \mathrm{E}-01$ & $-1.16 \mathrm{E}+05$ & $-2.48 \mathrm{E}+05$ & $2.25 \mathrm{E}+05$ & $1.45 \mathrm{E}-07$ & $-5.12 \mathrm{E}-08$ \\
\hline$-4.89 \mathrm{E}-02$ & $-5.64 \mathrm{E}-03$ & $1.11 \mathrm{E}-01$ & $-1.27 \mathrm{E}+05$ & $-6.41 E+05$ & $2.18 \mathrm{E}+05$ & $5.11 \mathrm{E}-08$ & $-2.29 \mathrm{E}-08$ \\
\hline$-6.29 \mathrm{E}-02$ & $1.13 \mathrm{E}-03$ & $5.09 \mathrm{E}+00$ & $-1.15 \mathrm{E}+06$ & $2.66 \mathrm{E}+05$ & $1.53 \mathrm{E}+06$ & $9.17 \mathrm{E}-07$ & $-2.09 \mathrm{E}-06$ \\
\hline$-2.76 \mathrm{E}-02$ & $-3.59 \mathrm{E}-02$ & $5.09 \mathrm{E}+00$ & $-3.48 \mathrm{E}+05$ & $2.14 \mathrm{E}+05$ & $9.26 \mathrm{E}+06$ & $1.45 \mathrm{E}-06$ & $-5.77 \mathrm{E}-03$ \\
\hline $9.61 \mathrm{E}-03$ & $2.88 \mathrm{E}-04$ & $5.09 \mathrm{E}+00$ & $-1.99 \mathrm{E}+05$ & $-3.14 \mathrm{E}+05$ & $2.52 \mathrm{E}+06$ & $1.82 \mathrm{E}-06$ & $-2.53 \mathrm{E}-05$ \\
\hline $3.05 \mathrm{E}-02$ & $4.05 \mathrm{E}-03$ & $5.09 \mathrm{E}+00$ & $9.67 \mathrm{E}+04$ & $-1.21 \mathrm{E}+05$ & $1.96 \mathrm{E}+07$ & $7.15 \mathrm{E}-07$ & $-1.15 \mathrm{E}-06$ \\
\hline $3.20 \mathrm{E}-02$ & $2.07 \mathrm{E}-03$ & $5.09 \mathrm{E}+00$ & $7.33 \mathrm{E}+04$ & $2.62 \mathrm{E}+05$ & $1.03 \mathrm{E}+07$ & $1.17 \mathrm{E}-06$ & $-6.60 \mathrm{E}-05$ \\
\hline$-3.10 \mathrm{E}-02$ & $3.87 \mathrm{E}-02$ & $5.09 \mathrm{E}+00$ & $-2.73 \mathrm{E}+05$ & $5.75 \mathrm{E}+05$ & $8.72 \mathrm{E}+06$ & $1.04 \mathrm{E}-06$ & $-4.31 \mathrm{E}-06$ \\
\hline $3.97 \mathrm{E}-02$ & $-2.92 \mathrm{E}-02$ & $5.09 \mathrm{E}+00$ & $-3.74 \mathrm{E}+04$ & $-4.85 \mathrm{E}+05$ & $1.50 \mathrm{E}+07$ & $1.05 \mathrm{E}-06$ & $-1.08 \mathrm{E}-06$ \\
\hline$-1.93 \mathrm{E}-02$ & $4.61 \mathrm{E}-02$ & $3.49 \mathrm{E}-01$ & $-2.80 \mathrm{E}+05$ & $3.41 \mathrm{E}+05$ & $1.11 \mathrm{E}+06$ & $1.29 \mathrm{E}-07$ & $-1.50 \mathrm{E}-08$ \\
\hline $2.13 \mathrm{E}-02$ & $-2.60 \mathrm{E}-02$ & $5.09 \mathrm{E}+00$ & $1.30 \mathrm{E}+05$ & $-2.14 \mathrm{E}+05$ & $1.94 \mathrm{E}+07$ & $7.88 \mathrm{E}-07$ & $-3.53 \mathrm{E}-06$ \\
\hline $2.13 \mathrm{E}-02$ & 4.47E-02 & $5.09 \mathrm{E}+00$ & $6.47 \mathrm{E}+04$ & $3.06 \mathrm{E}+05$ & $1.13 \mathrm{E}+07$ & $1.24 \mathrm{E}-06$ & $-1.24 \mathrm{E}-04$ \\
\hline $3.88 \mathrm{E}-02$ & $-3.03 \mathrm{E}-02$ & $4.58 \mathrm{E}+00$ & $2.94 \mathrm{E}+04$ & $-9.53 \mathrm{E}+04$ & $8.51 \mathrm{E}+05$ & $9.81 \mathrm{E}-07$ & $-2.97 \mathrm{E}-05$ \\
\hline $4.01 \mathrm{E}-02$ & $2.71 \mathrm{E}-02$ & $5.09 \mathrm{E}+00$ & $3.97 \mathrm{E}+05$ & $1.26 \mathrm{E}+05$ & $1.83 \mathrm{E}+07$ & $8.61 \mathrm{E}-07$ & $-6.67 \mathrm{E}-06$ \\
\hline$-5.85 \mathrm{E}-04$ & $-4.99 \mathrm{E}-02$ & $3.15 \mathrm{E}-01$ & $-1.27 \mathrm{E}+05$ & $-3.22 \mathrm{E}+05$ & $5.79 \mathrm{E}+05$ & $1.29 \mathrm{E}-07$ & $-1.07 \mathrm{E}-07$ \\
\hline$-4.94 \mathrm{E}-02$ & $-2.79 \mathrm{E}-03$ & $4.32 \mathrm{E}+00$ & $-1.33 \mathrm{E}+05$ & $2.86 \mathrm{E}+05$ & $1.01 \mathrm{E}+06$ & $1.10 \mathrm{E}-06$ & $-3.02 \mathrm{E}-05$ \\
\hline$-4.46 \mathrm{E}-02$ & $-2.18 \mathrm{E}-02$ & $5.00 \mathrm{E}+00$ & $5.88 \mathrm{E}+05$ & $6.08 \mathrm{E}+05$ & $3.69 \mathrm{E}+05$ & $9.58 \mathrm{E}-07$ & $-5.95 \mathrm{E}-06$ \\
\hline $2.89 \mathrm{E}-03$ & $2.82 \mathrm{E}-03$ & $5.09 \mathrm{E}+00$ & $9.96 \mathrm{E}+04$ & $-3.29 \mathrm{E}+04$ & $2.23 \mathrm{E}+07$ & $5.41 \mathrm{E}-07$ & $-1.29 \mathrm{E}-08$ \\
\hline$-1.33 \mathrm{E}-02$ & $3.94 \mathrm{E}-02$ & $5.09 \mathrm{E}+00$ & $-2.97 \mathrm{E}+05$ & $4.50 \mathrm{E}+05$ & $9.47 \mathrm{E}+06$ & $1.54 \mathrm{E}-06$ & $-3.08 \mathrm{E}-04$ \\
\hline
\end{tabular}

For analyzing the output file refer to $Z$ final values if it is observed that the ray travels all the way from beginning to end of the channel. Currents of those rays 
should be summed us as final current value. Dividing this value with some of all rays input current gives us the gain of the channel. 


\section{APPENDIX 4}

\section{CHARGED PARTICLE OPTICS (CPO) CODE FOR TILTED CHANNEL SIMULATION}

CPO3DS, 82nd example file, channel electron multiplier, tilted channels temp82.dat name of hidden output file, for processed data temp82b.dat name of main output data file, for ray data $\mathrm{m} \mathrm{n} / \mathrm{p} / \mathrm{m} / \mathrm{a}$ for segment printing level

0100 voltage reflection symmetries in $\mathrm{x}, \mathrm{y}, \mathrm{z}, \mathrm{x}=\mathrm{y}$ planes 2 number of different voltages, time dependence

0.00120 allowed consistency error, side/length ratio check $\mathrm{n}$ apply inscribing correction $(\mathrm{a} / \mathrm{s} / \mathrm{n}=$ always/sometimes/never) ect -end con or cyl triangle *** $-0.003061940-0.01736512$ corner 1

$0.04770942-0.05-0.01736497$ corner 2 $-0.001530970-0.00868256$ corner 3 $0.047709420-0.01736497$ centre of cut bounding plane 1e-08 angle of cone $-868240.839094260-4924038.77374345$ point of cone $0.049240390-0.00868241$ any other point on axis in direction of opening 12 numbers of 2 applied voltages (can be same) $-0.01694 .924 \quad$ z's of application points

32 number of subdivisions ect -end con or cyl triangle $* * *$ 
$0.04770942-0.05-0.01736497$ corner 1

$0.098480780-0.01736482$ corner 2

$0.04924039-0.05-0.00868241 \quad$ corner 3

$0.047709420-0.01736497$ centre of cut bounding plane

1e-08 angle of cone

$-868240.839094260-4924038.77374345$ point of cone

$0.049240390-0.00868241$ any other point on axis in direction of opening

12 numbers of 2 applied voltages (can be same)

-0.01694 .924 z's of application points

32 number of subdivisions

cre -rectangle on cylinder $* * *$

$-0.001530970-0.00868256 \quad$ corner 1

$0.04770942-0.05-0.01736497$ corner 2

$0.04924039-0.05-0.00868241 \quad$ corner 3

$000 \quad$ corner 4

0.05 radius

$0.047709420-0.01736497$ first point on axis

$0.049240390-0.00868241$ second point on axis

12 numbers of 2 applied voltages (can be same)

$-0.01694 .924 \quad z$ 's of application points

900 total $\mathrm{nr}$ of subdivs and 0 , or subdivs along and around axis

cylindrical electrode $\quad * * *$ main part of channel

$0.050 .049240390-0.00868241$ radius, centre of 1 st end 
0.057922800 .04055798 centre of 2 nd end

12 numbers of 2 applied voltages (can be same)

$-0.01694 .924 \quad$ z's of application points

108 total $\mathrm{nr}$ of subdivs and 0 , or subdivs along and around axis

cylindrical electrode $\quad * * *$ main part of channel

0.050 .057922800 .04055798 radius, centre of 1 st end

0.9087988704 .86611597 centre of 2 nd end

12 numbers of 2 applied voltages (can be same)

$-0.01694 .924 \quad$ z's of application points

1008 total $\mathrm{nr}$ of subdivs and 0 , or subdivs along and around axis

cylindrical electrode $\quad * * *$ main part of channel

0.050 .9087988704 .86611597 radius, centre of 1st end

0.9174812804 .91535636 centre of 2 nd end

12 numbers of 2 applied voltages (can be same)

$-0.01694 .924 \quad$ z's of application points

108 total $\mathrm{nr}$ of subdivs and 0 , or subdivs along and around axis

ect -end con or cyl triangle ***

0.969783604 .92403906 corner 1

0.919012250 .054 .92403891 corner 2

0.9682526304 .9153565 corner 3

0.9190122504 .92403891 centre of cut bounding plane

1e-08 angle of cone

868241.8058159304924043 .6804174 point of cone 
0.9174812804 .91535636 any other point on axis in direction of opening

12 numbers of 2 applied voltages (can be same)

$-0.01694 .924 \quad$ z's of application points

32 number of subdivisions

ect -end con or cyl triangle ***

$0.919012250 .054 .92403891 \quad$ corner 1

0.8682408904 .92403877 corner 2

0.917481280 .054 .91535636 corner 3

0.9190122504 .92403891 centre of cut bounding plane

1e-08 angle of cone

868241.8058159304924043 .6804174 point of cone

0.9174812804 .91535636 any other point on axis in direction of opening

12 numbers of 2 applied voltages (can be same)

$-0.01694 .924 \quad$ z's of application points

32 number of subdivisions

cre -rectangle on cylinder $* * *$

0.9682526304 .9153565 corner 1

$0.919012250 .054 .92403891 \quad$ corner 2

0.917481280 .054 .91535636 corner 3

0.9667216604 .90667395 corner 4

0.05 radius

0.9190122504 .92403891 first point on axis

0.9174812804 .91535636 second point on axis 
12 numbers of 2 applied voltages (can be same)

-0.01694 .924 z's of application points

900 total $\mathrm{nr}$ of subdivs and 0 , or subdivs along and around axis

edi evenly-divided disc $\quad * * *$ Source disc

$0.0750 .0481470-0.066941$ radius, centre of disc

$0.0481470-0.016941$ any other point on axis

11 numbers of 2 applied voltages (can be same)

10 number of subdivisions

cylindrical electrode $\quad * * *$ Shield

$0.0750 .0481470-0.066941$ radius, centre of 1 st end

$0.0481470-0.016941$ centre of 2 nd end

11 numbers of 2 applied voltages (can be same)

58 total $\mathrm{nr}$ of subdivs and 0 , or subdivs along and around axis

disc, uneven radial division ${ }^{* * *}$ surround of source end of channel

$0.050 .0481470-0.016941 \quad 1$ st radius, centre of disc

0.0750 .04814700 .9830592 2nd radius, any other point on axis

11 numbers of 2 applied voltages (can be same)

38 total $\mathrm{nr}$ of subdivs and 0 , or subdivs along radius and around axis

disc, uneven radial division ***surround of end of channel

$0.050 .91904 .924 \quad 1$ st radius, centre of disc

$0.0750 .9190-0.0762$ 2nd radius, any other point on axis

22 numbers of 2 applied voltages (can be same)

38 total $\mathrm{nr}$ of subdivs and 0 , or subdivs along radius and around axis 
cylindrical electrode $\quad * * *$ shield

0.0750 .91904 .924 radius, centre of 1 st end

0.91904 .974 centre of 2 nd end

22 numbers of 2 applied voltages (can be same)

58 total $\mathrm{nr}$ of subdivs and 0 , or subdivs along and around axis

edi evenly-divided disc $\quad * * *$ anode

0.0750 .91904 .974 radius, centre of disc

$0.9190-0.076$ any other point on axis

22 numbers of 2 applied voltages (can be same)

10 number of subdivisions

end of electrode information

$\begin{array}{llll}0 & 0 & 0.5 & \mathrm{x}\end{array}$ final nmbr segments, nmbr steps, weight, disable overlap test

1e-07 charge inaccuracy, zero total charge

end of segment information

$0.0000000 \mathrm{E}+00 \quad$ cathode

$1.5000000 \mathrm{E}+03$

n no more magnetic fields from menu

$\mathrm{n} \mathrm{n} \mathrm{n}$ no more potentials and fields along a line

start of ray information

d direct (d), or mesh (m) method and mesh spacing

$\mathrm{p}$ 'n/p/m/a' for 'nearlyzero/partial/most/all' printing level, rho/radius

-0.081 .0 minimum and maximum $\mathrm{x}(\mathrm{mm})$ of screen $2 \mathrm{D}$ fields of view

-0.060 .06 minimum and maximum $\mathrm{y}$ 
-0.08 5.0 minimum and maximum $\mathrm{z}$

-0.13 minimum and maximum $\mathrm{x}(\mathrm{mm})$ of rays

-0.1 0.1 minimum and maximum y

-0.1 4.97 minimum and maximum $\mathrm{z}$

$-1 \mathrm{e}+101 \mathrm{e}+10$ minimum and maximum $\mathrm{vx}(\mathrm{m} / \mathrm{s})$ of rays

$-1 \mathrm{e}+101 \mathrm{e}+10$ minimum and maximum vy

$-1 \mathrm{e}+101 \mathrm{e}+10 \quad$ minimum and maximum $\mathrm{vz}$

-0.55 minimum and maximum rho $\left(=\operatorname{sqrt}\left(\mathrm{x}^{* *} 2+\mathrm{y}^{* * 2}\right)\right)$

$+\mathrm{s}$ direction of time, secondaries option

rm $1111520-0.5-2504$ seed, min en, kT, add, mult

2e-05 final time (ms)

0.010 .010 initial and max step length, interpolation points

-0.0001 1e-05 fractional inaccuracies for (1) ray tracing and (2) potentials and fields

1 nr test planes, mult cross, iter foc,phase spc,scatt,quant

0014.96 a,b,c,d of test plane defined by $a^{*} x+b^{*} y+c^{*} z=d$

el 'el' for electron; or 'co' or 'va' for other particles

k kinetic energy $(\mathrm{k})$; or total energy $(\mathrm{t})$ and potential

cylindrical beam

e edge distribution, non-random

$0.0481470-0.0570$ centre and radius of window

0.04814700 .005 any other point on axis

0.04814700 .0030 .05 centre and radius of pupil

$1201001 \mathrm{e}-06$ n's for source disc and pupil, eV, mA 
n calculate space-charges?

$00000 \quad$ symmetries of rays in yz,zx,xy and $\mathrm{x}=\mathrm{y}$ planes

CPO3DS, 82nd example file, channel electron multiplier, tilted channels. (See xmpl3d81 for non-tilted channels.)

This example is essentially the same as xmpl3d 81 except that the channel is tilted by 10 degrees. Further examples of tilts of 5 to 25 degrees are given at the end of the present file, and the results from them are presented below.

One channel is simulated. It has diameter $0.1 \mathrm{~mm}$, length $5 \mathrm{~mm}$. The 2 ends are shielded with small field-free boxes. The voltage difference between the ends is $1500 \mathrm{~V}$. The channel is tilted by 10 degrees. The procedure used to create this file is described below. 20 electrons are injected with an energy of $100 \mathrm{eV}$.

See $\mathrm{xmpl3d} 81$ for a description of the simulation of the secondary production.

The 'minimum incident energy' for collisions is given the value $5 \mathrm{eV}$, so if a primary ray has an energy smaller than this the ray is stopped. This is the main cause of rays not reaching the anode.

3 of the 20 rays reach the anode at the end of the channel (but this small number is a statistical result, the fraction that reach the anode is higher when more rays are used, see below). Using the analysis file given at the end of xmpl3d81.dat:

Number of rays $=20$

Number reaching anode $=3$

Number stopped because secondaries are not allowed

to be produced more than 29 times $=0$

Total number of collisions $=153$ 
Total of final ray currents $=-5.614 \mathrm{E}-03$

Total current at test plane $=-1.003 \mathrm{E}-03$

Highest single current $=-8.786 \mathrm{E}-04$

The initial total current of the 20 rays is $5 . \mathrm{E}-7 \mathrm{~mA}$.

Therefore the gain is approximately 2000 . But the statistical error is high with only

20 rays (in the present case, very high).

As given below, when 500 rays are used the fraction that reaches the anode is $43 \%$ and the gain is 311,000 .

\section{RESULTS FOR VARIOUS TILTED CHANNELS}

Analyses are with AnMCPf90.f90, given at end of xmpl3d81.dat.

\section{TILT ANGLE 0 DEGREES}

Number of rays $=500$

Number reaching anode $=332$

Number of collisions $=4612$

Total current $=-3.302 \mathrm{E}-02$

Test plane current $=-3.111 \mathrm{E}-02$

Highest current $=-3.499 \mathrm{E}-03$

TILT ANGLE 5 DEGREES

Number of rays $=500$

Number reaching anode $=313$

Number stopped because secondaries are not allowed to be produced more than 29 times $=0$ 
Total number of collisions $=5153$

Total of final ray currents $=-9.768 \mathrm{E}-02$

Total current at test plane $=-9.378 \mathrm{E}-02$

Highest single current $=-8.708 \mathrm{E}-03$

\section{TILT ANGLE 10 DEGREES}

Number of rays $=500$

Number reaching anode $=217$

Number stopped because secondaries are not allowed

to be produced more than 29 times $=1$

Total number of collisions $=5252$

Total of final ray currents $=-1.604 \mathrm{E}-01$

Total current at test plane $=-1.555 \mathrm{E}-01$

Highest single current $=-3.775 \mathrm{E}-02$

TILT ANGLE 15 DEGREES

Number of rays $=500$

Number reaching anode $=87$

Number stopped because secondaries are not allowed

to be produced more than 29 times $=8$

Total number of collisions $=5044$

Total of final ray currents $=-1.847 \mathrm{E}-01$

Total current at test plane $=-1.558 \mathrm{E}-01$

Highest single current $=-3.406 \mathrm{E}-02$ 


\section{TILT ANGLE 20 DEGREES}

Number of rays $=500$

Number reaching anode $=5$

Number stopped because secondaries are not allowed

to be produced more than 29 times $=8$

Total number of collisions $=4221$

Total of final ray currents $=-2.840 \mathrm{E}-02$

Total current at test plane $=-1.690 \mathrm{E}-03$

Highest single current $=-1.570 \mathrm{E}-03$

\section{TILT ANGLE 25 DEGREES}

(but max z was 5.07 instead of 4.60, so extra collisions)

(segs at ends of main cyl not concentrated)

Number of rays $=500$

Number reaching anode $=0$

Total number of collisions $=2514$

Total of final ray currents $=-2.970 \mathrm{E}-05$

Total current at test plane $=0.000 \mathrm{E}+00$

Highest single current $=0.000 \mathrm{E}+00$

\section{COMMENTS ON RESULTS}

A similar, but more limited, simulation of short channels has been described by Y.

S. Choi and J. M. Kim, Monte Carlo simulations for tilted-channel electron multipliers, IEEE Transactions on Electron Devices, Vol 47, No, 6, June 2000, 1293 1296. They found that the multiplication factor increases as the tilt angle is increased, 
reaching a maximum at an angle of approximately 25 degrees, and decreasing rapidly at higher angles. They noted that when the channel is tilted the rays tend to collide repeatedly with the higher-voltage side of the channel and suggest that this is the reason for the increase in multiplication. We find in the present simulations that in the non-tilted channel many of the rays travel near the axis of the channel for a substantial fraction of the channel length between collisions, particularly after their last collision before reaching the anode. We also find in the present simulation that when the channel is tilted the rays tend to collide more frequently with the highervoltage side than with the low-voltage side and that there are no rays that travel near the axis for long distances. The highest multiplication factor occurs at approximately 15 degrees, and is then larger than that at 0 degrees by a factor of approximately 5. At higher tilt angles the gain decreases sharply.

\section{PROCEDURE FOR SETTING UP A TILTED CHANNEL}

(1) Start by making a copy of xmpl3d82.dat (called temp.dat, say).

(2) Search temp.dat for the electrode called 'Source' and the 5 following electrodes called 'shield', 'surround', 'surround', 'shield' and 'anode'. We shall refer to these 6 electrodes as the 'field-free boxes'.

(3) Keep the field-free boxes but delete all the other electrodes.

(4) Exit from temp.dat, find prog26.dat and copy it to tempin.dat.

(5) Edit tempin.dat so that it has the form:

$0.05 \quad !=$ radius of cylinder

$0.008749 \quad !=$ highest $\mathrm{z}$ of cut cylinder (called zm below)

$8 \quad !=$ subdivision number around axis 
$100 !$ ! =approximate total number of segments

$1 \quad !=$ number of the applied voltage

(6) These data control the size and shape of the required wedge-shaped ends of the tilted channel. The data above were used in the construction of xmpl3d82.dat. Replace the radius by your required radius. Replace the 'highest $\mathrm{z}$ of cut cylinder' by the number $2 * \mathrm{r} * \tan ($ theta), where theta is the required angle of inclination.

(7) Find the executable file 'prog26' and run it. It reads from tempin.dat and produces the file tempout.dat, which holds the data for the electrodes of the wedgeshaped ends.

(8) Paste the contents of tempout.dat into the electrode area of temp.dat.

(9) Run CPO3DS on temp.dat but stop the ray tracing as soon as it starts. On the screen you will see the field-free boxes and a wedge-shaped end. Do not worry about their positions at this stage in the procedure.

(10) Find the numbers of the electrodes that make up the wedge-shaped end (perhaps 1, 2 and 3). Use the option /data builder/electrodes/transform/ to shift these electrodes by an arbitrary distance (say $4 \mathrm{~mm}$ ) in the $+\mathrm{z}$ direction. Do not shift the field-free boxes.

(11) Again paste the contents of tempout.dat into the electrode area of temp.dat.

(12) Again run CPO3DS on temp.dat. On the screen you will now see the field-free boxes and 2 wedge-shaped ends.

(13) Use the transform option to reflect the latest, left-hand, wedge in the $\mathrm{z}=0$ plane and then separately to rotate it by 180 degrees about the $\mathrm{z}$ axis. 
(14) Now insert a cylinder that has the required radius and length and that has its axis along the $z$ axis, starting at $z=0$. It is recommended (as in $x m p 13 d 82$ ) that the cylinder should be in 3 parts, with the short end parts having more axial divisions than the main middle part.

(15) Use the transform shifting option to place the wedge-shaped ends as nearly as possible at the ends of the cylinder, centered on the $\mathrm{z}$ axis. Find the required shifting distances by zooming into a relevant region and using the cursor to find the relevant coordinates.

(16) The cylinder and its ends now have to be rotated together about the $y=0$ axis by the required tilt angle. Do not move the field-free boxes yet.

(17) Now shift the field-free boxes to their correct positions.

(18) The tilted channel is now ready but voltage number 1 is at present applied to all the electrodes except the electrodes of the right-hand field-free box, which have the correct voltage number 2 . The left-hand field-free box also has the correct voltage number 1. All the other electrodes need the two applied voltages 1 and 2 , to give them a voltage gradient.

(19) The two applied voltages are 0 and the anode voltage (perhaps 1000 or 1500). We now need to find the "z's of application points" of these two voltages. The first voltage is applied at the $\mathrm{z}$ of the 'surround' of the left-hand field-free box. Similarly the second voltage is applied at the $\mathrm{z}$ of the 'surround' of the right-hand field-free box.

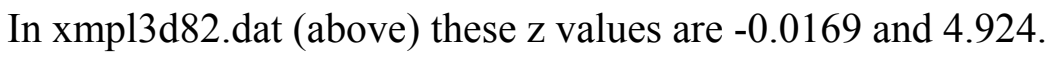

(20) Use these values for all the electrodes of the cylinder and the wedge-shaped ends. 
(21) Finally set up the source of electrons. Find the centre of the electrode labeled 'Source'. This is a suitable position for the source of the primary electrons (a more suitable position, as in $\mathrm{xmpl3d} 82$, is a short distance away from the 'Source' electrode).

(22) There are of course many other parameters that you can change, but those already in xmpl3d82.dat and temp.dat are suitable for an initial study. 
APPENDIX 5

LABVIEW BLOCK DIAGRAMS

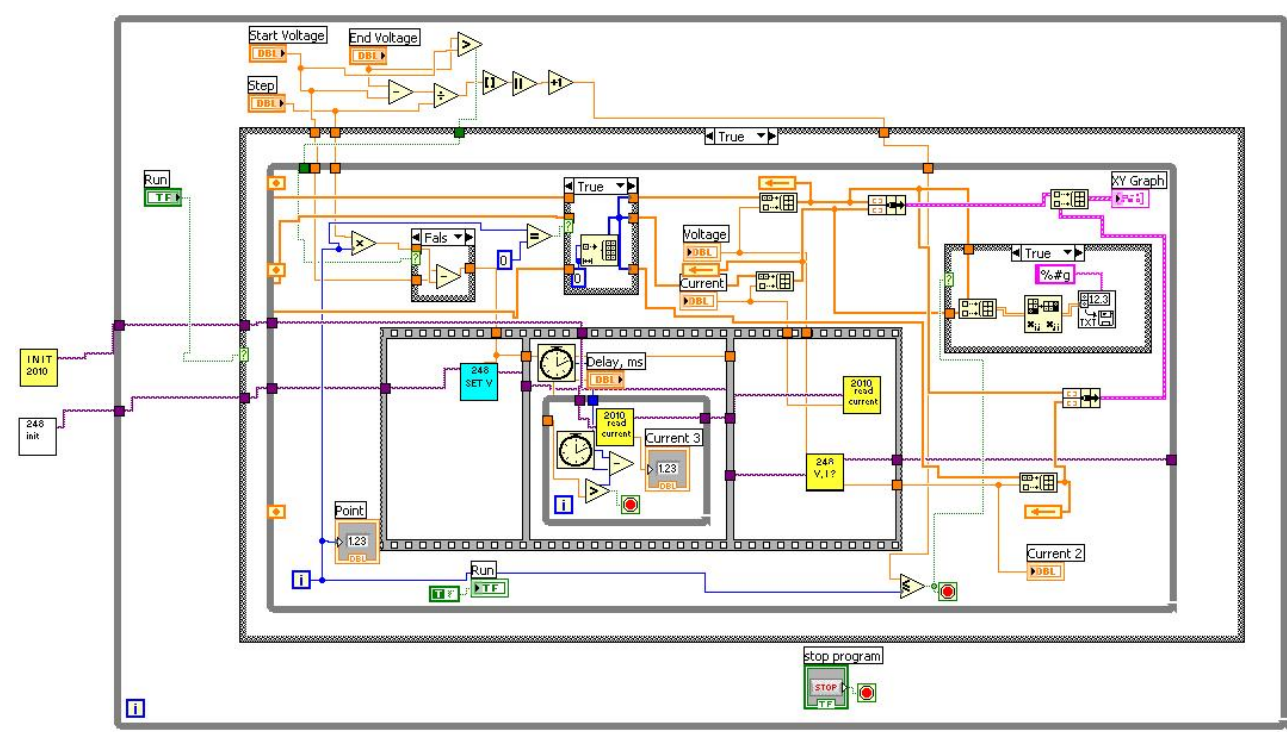

Block diagram for I-V program.

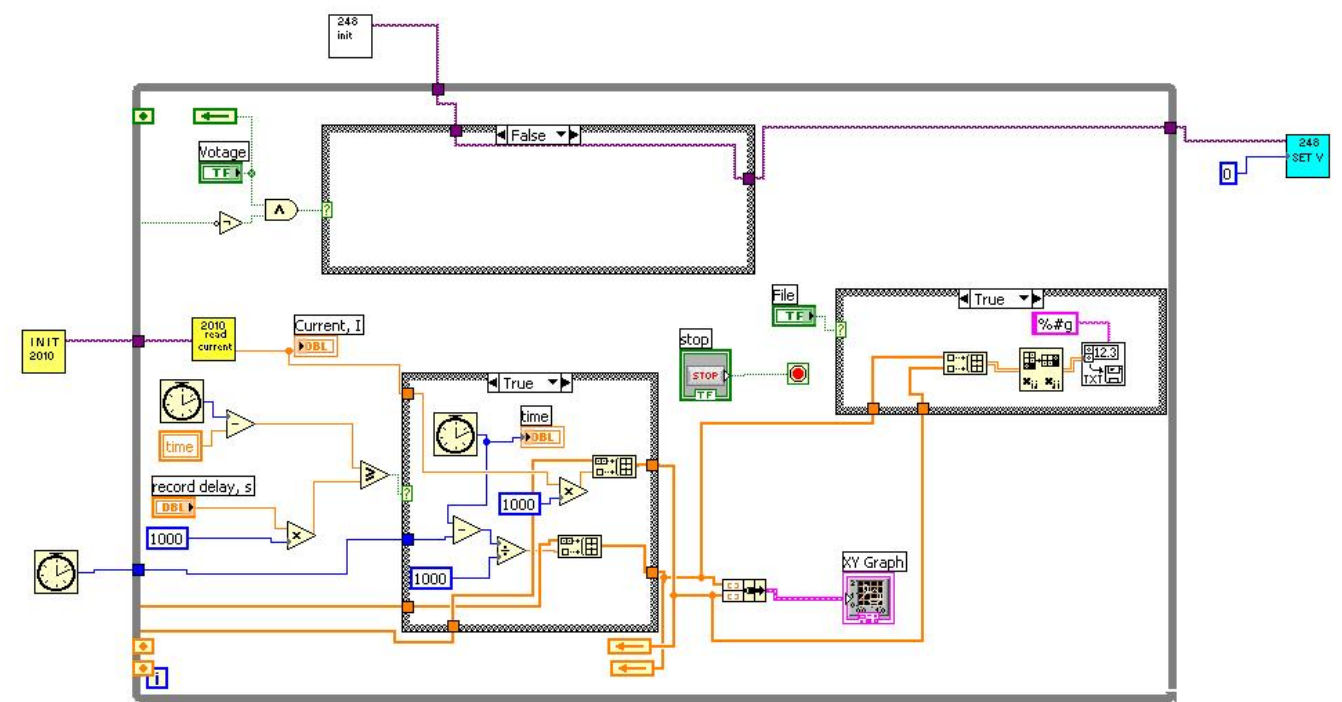

Block diagram for emission stability program. 


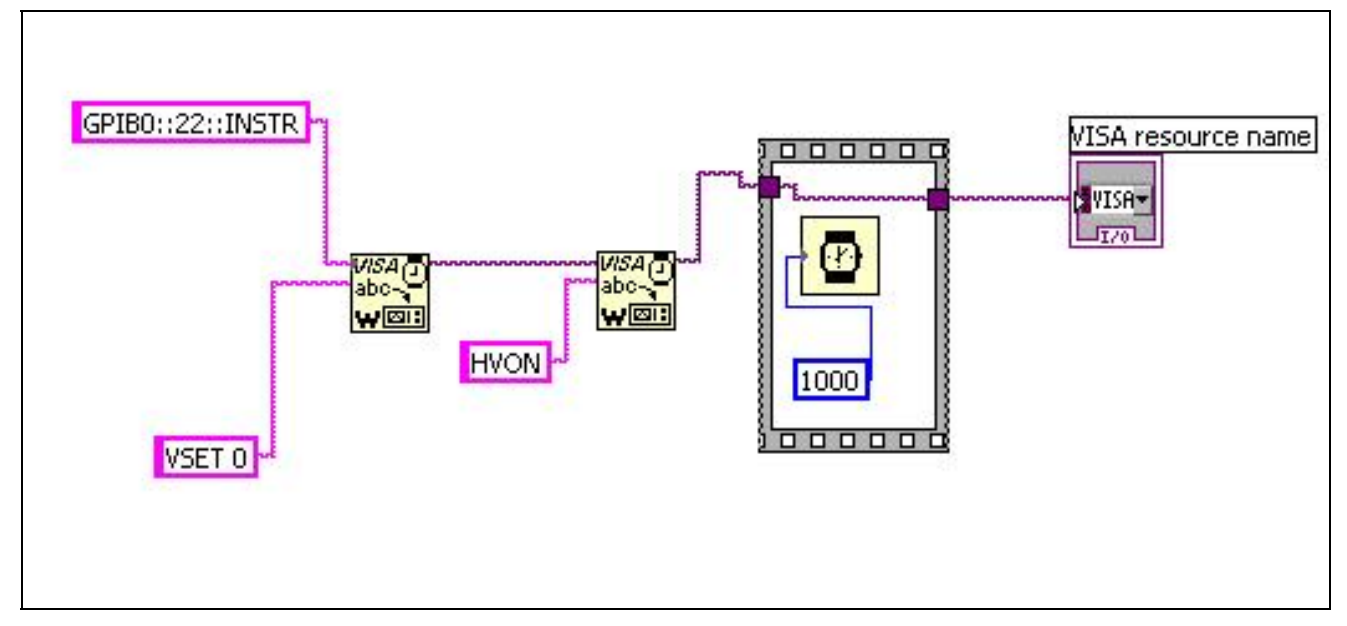

Block diagram for initialization voltage source 248 .

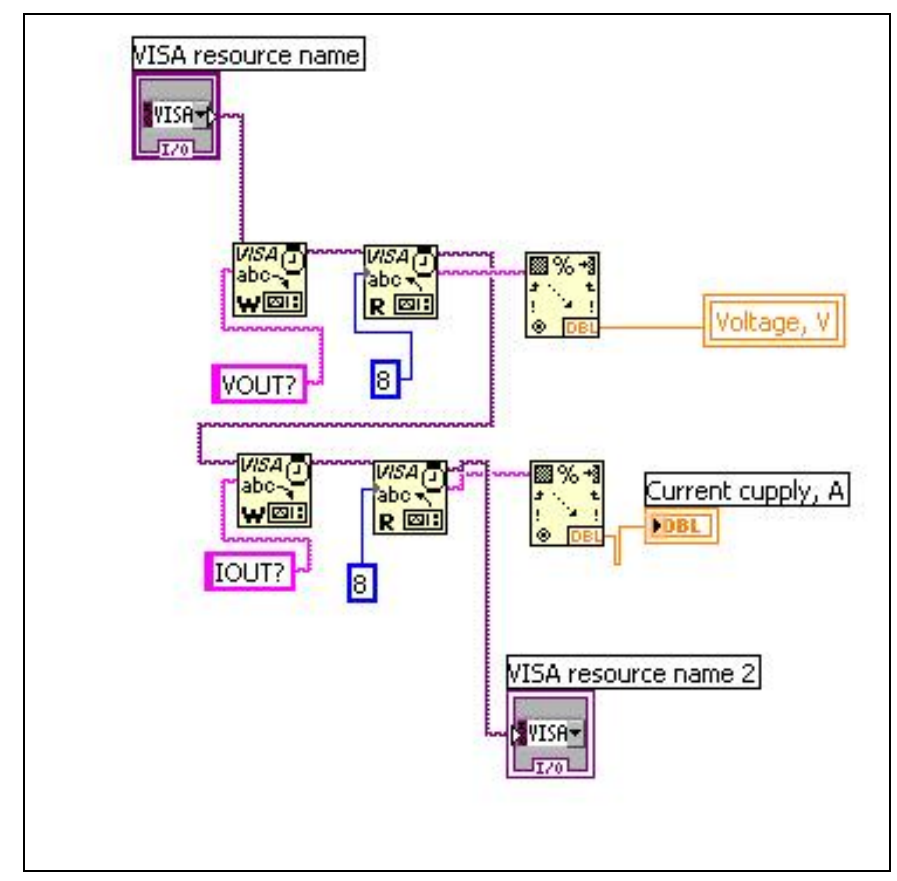

Block diagram for reading voltage from source 248 . 


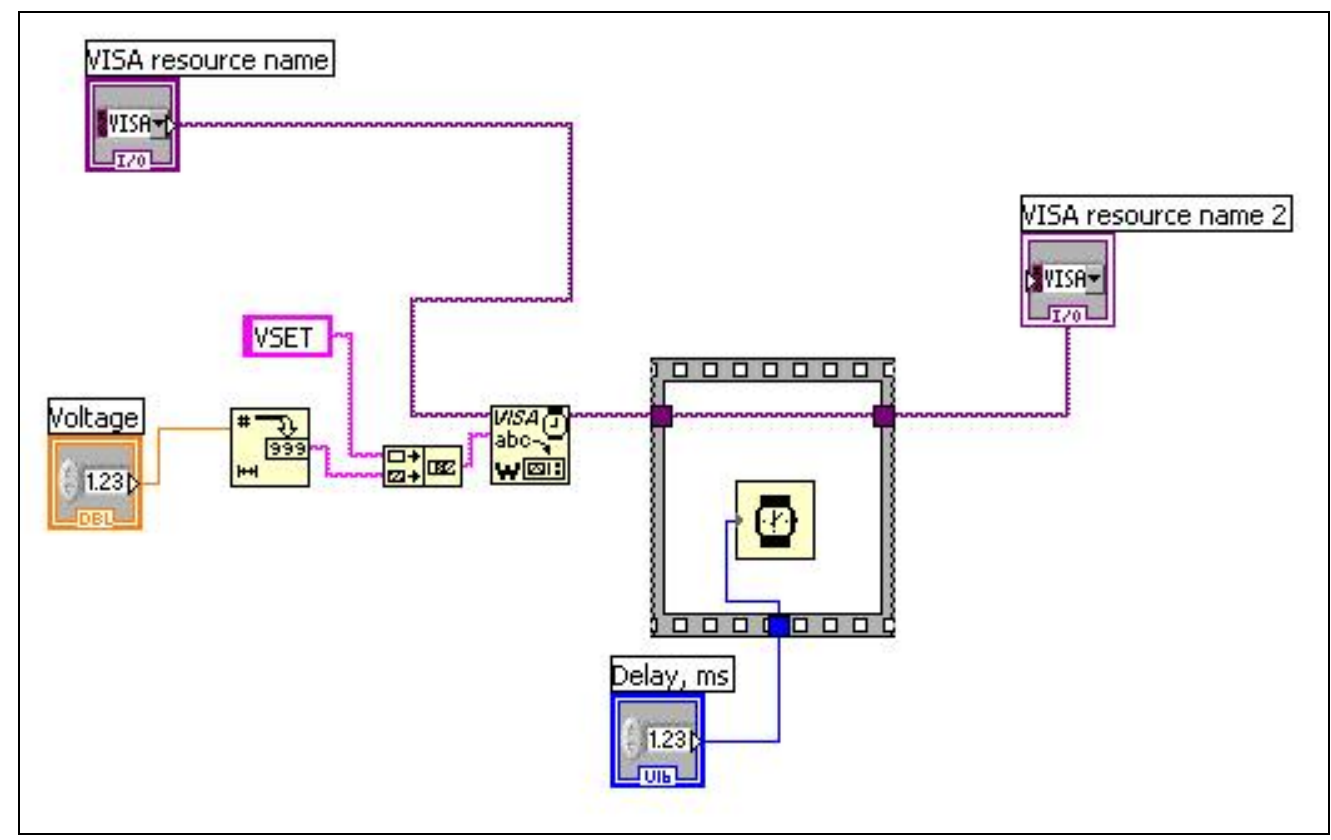

Block diagram for setting voltage on source 248 .

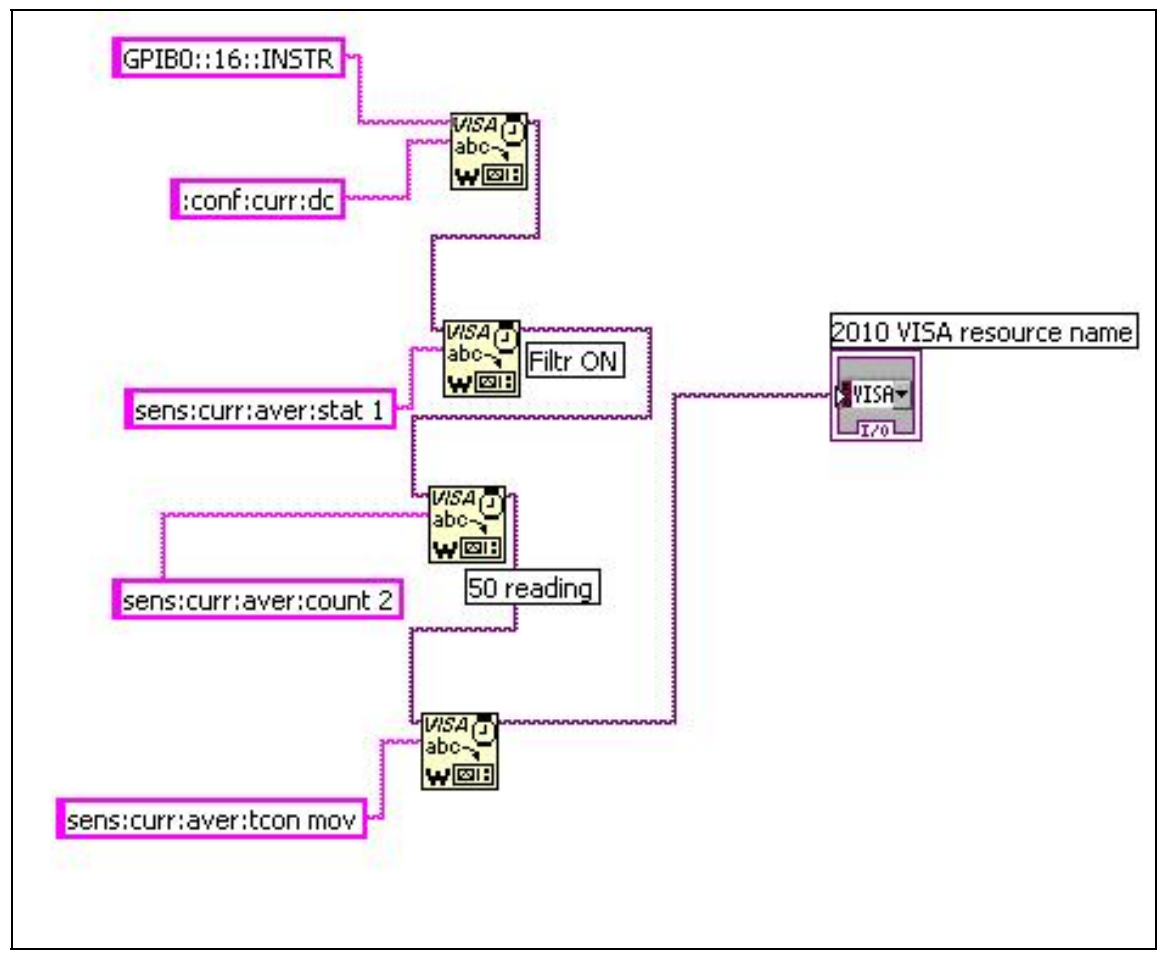

Block diagram for initialization of 2010 . 


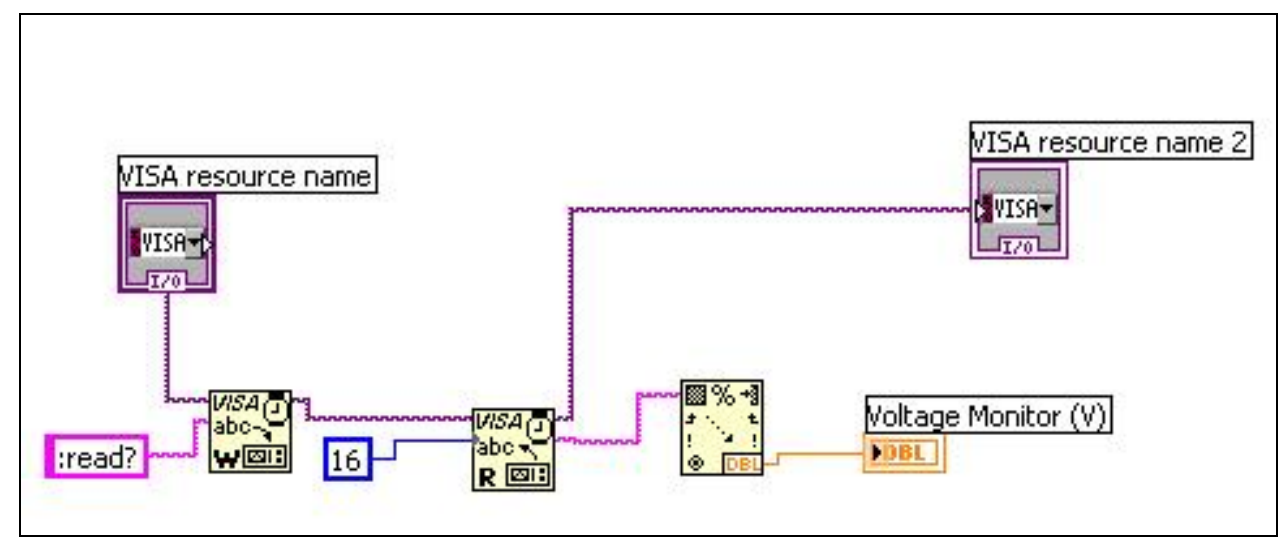

Block diagram for reading voltage from 2010. 


\title{
Lattice Dynamics of $\mathrm{MgO}$ at High Pressure: Theory and Experiment
}

\author{
Subrata Ghose, ${ }^{1}$ Michael Krisch, ${ }^{2}$ Artem R. Oganov, ${ }^{3}$ Alexandre Beraud, ${ }^{2}$ Alexey Bosak, ${ }^{2}$ Revansidha Gulve, ${ }^{4}$ \\ Raghunandan Seelaboyina, ${ }^{4}$ Hexiong Yang, ${ }^{4}$ and Surendra K. Saxena ${ }^{4}$ \\ ${ }^{1}$ Mineral Physics Group, Box 351310 , University of Washington, Seattle, Washington 98195, USA \\ ${ }^{2}$ European Synchrotron Radiation Facility, Boite Postale 220, F-38043 Grenoble Cedex, France \\ ${ }^{3}$ Laboratory of Crystallography, Department of Materials, ETH Zurich, CH-8093 Zurich, Switzerland \\ ${ }^{4}$ Center for Study of Matter at Extreme Conditions, Florida International University Park, VH-140, Miami, Florida 33199, USA \\ (Received 27 February 2005; published 26 January 2006) \\ The longitudinal acoustic and optical phonon branches along the $\Gamma-X$ direction of $\mathrm{MgO}$ at $35 \mathrm{GPa}$ have \\ been determined by inelastic $x$-ray scattering using synchrotron radiation and a diamond-anvil cell. The \\ experimentally observed phonon branches are in remarkable agreement with $a b$ initio lattice dynamics \\ results. The derived themodynamic properties, such as the specific heat $C_{V}$ and the entropy $S$ are in very \\ good accord with values obtained from a thermodynamically assessed data set involving measured data on \\ molar volume, heat capacity at constant pressure, bulk modulus and themal expansion.
}

The experimental determination of the phonon dispersion at high pressure constitutes an important ingredient for the characterization of the physical properties of materials in extreme conditions. It gives access to valuable quantitative information conceming elasticity, thermodynamic properties, and the dynamics of phase instabilities. Furthermore, the experimental data provide important tests for the accuracy of theoretical lattice dynamical models. Among these the most advanced ones are $a b$ initio quantum mechanical calculations, using density-functional perturbation theory. Critical inputs are the appropriate choice of the potential (all-electron or pseudopotential approaches) and the correct description of the exchangecorrelation term. If a good agreement with the experimental phonon dispersion is observed, these calculations can then be used with increased confidence to describe the physical properties such as elastic and dielectric constants, etc., and thermodynamic properties, such as specific heat, entropy, etc., at very high pressures beyond the reach of current experimental methods.

Until recently, inelastic neutron scattering (INS) has been extensively used to study the phonon dispersion throughout the Brillouin zone of the crystal. Phonon dispersion has been determined in a large number and variety of crystals by INS as well as their temperature dependence, particularly in connection with phase transitions and soft modes, i.e., phonons whose frequency approaches zero as $T$ approaches the transition temperature $T_{c}$. However, the weak interaction of neutrons with matter and the typical size of neutron beams require the use of relatively large (ca. $1 \mathrm{~cm}^{3}$ ) single crystals, which puts an upper limit for the measurement of phonon dispersion at high pressure at present to about 10-15 GPa by INS [1]. The technique of inelastic $x$-ray scattering (IXS) in conjunction with third generation synchrotron sources now makes it possible to measure the phonon dispersion at high pressures up to about $50 \mathrm{GPa}$ in crystals tens of microns in size mounted in a diamond-anvil cell. Using $\mathrm{He}$ as a pressure transmitting medium, the phonon dispersions in single crystals of Ar to $20 \mathrm{GPa}$ [2] and in hcp-Co to $39 \mathrm{GPa}$ [3] have thus been recorded. Without a pressure transmitting medium, pressures as high as $112 \mathrm{GPa}$ were reached on polycrystalline samples of iron and iron containing compounds [4,5].

In this Letter, we present the experimental determination of the longitudinal acoustic (LA) and optical (LO) phonon branches along the $\Gamma-X$ direction in $\mathrm{MgO}$ at $35 \mathrm{GPa}$, utilizing inelastic $x$-ray scattering, and the comparison with $a b$ initio calculations. Our choice of $\mathrm{MgO}$ is motivated by its importance in various fields of research. The material is regarded as the prototype oxide due to its simple structure and the large stability field (in pressure and temperature) of the $\mathrm{NaCl}$ structure [6]. Moreover, it is considered as a pressure calibration standard for highpressure sciences [7]. Furthermore, $\mathrm{MgO}$ is an important ceramic for industrial applications, and of great interest for Earth sciences, since it is a major mineral phase of the Earth's lower mantle [8].

A doubly polished single crystal of $\mathrm{MgO}$ of (100) orientation, $30 \times 50 \mu \mathrm{m}$ size and a thickness of $20 \mu \mathrm{m}$ was loaded in a diamond-anvil cell with $\mathrm{He}$ as pressure transmitting medium along with a few very small fragments of ruby for the pressure calibration. The pressures were determined from the ruby $R_{1}$ fluorescence line and were accurate to $\pm 0.5 \mathrm{GPa}$. The IXS experiment was performed on beamline ID28 at the European Synchrotron Radiation Facility, Grenoble, France. $X$ rays from an undulator source were monochromatized by a silicon (111) double crystal and a backscattering very high-energy resolution monochromator, utilizing the silicon (999) reflection order at $17794 \mathrm{eV}$. A cylindrical mirror in conjunction with a dynamically bent multilayer provided a focus of $25 \times$ $60 \mu \mathrm{m} H$ (horizontal) $\times V$ (vertical) full width at half maximum (FWHM) at the sample position. The scattered 


\title{
Enhanced field emission of thin multiwall carbon nanotubes by electron multiplication from microchannel plate
}

\author{
Raghunandan Seelaboyina, Jun Huang, and Won Bong Choi ${ }^{\mathrm{a}}$ \\ Center for Nanomaterials and Devices, Department of Mechanical and Materials Engineering, \\ Florida International University, Miami, Florida 33174
}

(Received 2 March 2006; accepted 27 March 2006; published online 11 May 2006)

We report on a method to amplify electron emission from thin multiwall carbon nanotube (MWCNTs) field emitters using an electron multiplier microchannel plate. High density thin MWCNTs were synthesized by themal chemical vapor deposition. Density of thin MWCNTs was increased by decreasing the amount of $\mathrm{Al}_{2} \mathrm{O}_{3}$ in the catalyst solution. The high density of nanotubes led to their vertical orientation. The thin MWCNTs showed low turn-on electric field and high electron emissivity which could be attributed to their wall thinness and high aspect ratio $(\sim 1500)$ resulting in high field enhancement factor $(\sim 9300)$. Emission current was stable with an average fluctuation of $2 \%$. A microchannel plate positioned between cathode and anode increased the total emission current by 7.5 times. 12006 American Institute of Physics.

[DOI: $10.1063 / 1.2203218$ ]

Unique electrical and mechanical properties of carbon nanotubes (CNTs) have led to their investigation for application as electron sources in vacuum microelectronics, field emission displays, and microwave sources. ${ }^{1-4}$ Though thermionic cathodes are primarily used for these applications they have several disadvantages such as long turn-on time, poisoning of materials, high operation temperature, and high power consumption. Where as low threshold field for emission, instantaneous response to field variation, resistance to temperature fluctuation, high durability, high current density, ${ }^{5}$ and power efficiency of CNTs make them ideal candidate for future electron emitters.

In this letter we report a method for achieving high emission current from CNTs and electron multiplier microchannel plate (MCP). Microchannel plates are used in many applications: $\mathrm{X}$-ray, astronomy, e-beam fusion studies, nuclear science, ${ }^{6}$ and high efficiency field emission display. ${ }^{7}$ Our new approach of combining CNT emitters and MCP will provide several benefits. MCP amplifies the emission current and also protects the CNTs from irreversible damage during vacuum arcing. ${ }^{8} \mathrm{MCP}$ operates by avalanche multiplication of secondary electrons, which are generated when incident electrons strike the channel walls of a MCP. A voltage applied across the ends of the MCP creates a field which accelerates the secondary electrons along the channel leading to avalanche multiplication.

Jeong et al. ${ }^{9}$ recently reported the synthesis of randomly oriented thin multiwall carbon nanotubes (MWCNTs) (diameter: $10 \mathrm{~nm}$, catalysts: $\mathrm{Fe}-\mathrm{Mo}-\mathrm{MgO}$ ) by catalytic chemical vapor deposition. This study involves synthesis of vertically oriented thin MWCNTs by thermal chemical vapor deposition. The synthesized nanotubes had small diameters, large aspect ratio, and high field enhancement factor resulting in good field emission (FE) properties. Because of the intermediate structural characteristics between singlewall carbon nanotubes (SWCNTs) and MWCNTs, thin MWCNTs have been suggested as good candidates for field emission appli-

\footnotetext{
2) Author to whom correspondence should be addressed; electronic mail:
} choiw e fiu.edu cations. SWCNTs have high aspect ratio and small radius of curvature $(\sim 1 \mathrm{~nm})$ and have been reported to have large field enhancement factor, low threshold voltage, and high emission current. ${ }^{4}$ However, degradation of emission current with time is an obstacle for practical application of SWCNTs as field emitters in harsh environment. ${ }^{10}$ While MWCNTs have longer life time, their large diameter result in higher turn-on voltage and low current density with small field enhancement factor. ${ }^{11}$ The objective of this study was to demonstrate a method for achieving high emission current from CNT field emitters, which are robust enough to endure intense environments and operate at reasonable power levels. In our unique approach we have investigated the emission property of thin MWCNT emitters with MCP for field emission multiplication (Fig. 1).

The thin MWCNTs were synthesized by thermal chemical vapor deposition (CVD) on $\mathrm{Nb} / \mathrm{SiO}_{2} / \mathrm{Si}$ substrates from a catalyst solution having Mo doped Fe particles supported by $\mathrm{Al}_{2} \mathrm{O}_{3}$ nanoparticles. Catalyst solution was prepared from iron (III) nitrate-nonahydrate, bis(acetylacetonato)dioxomolybdenum(VI), and $\mathrm{Al}_{2} \mathrm{O}_{3}$ nanoparticles in methanol. Uniform suspension of catalyst in methanol with various amounts of $\mathrm{Al}_{2} \mathrm{O}_{3}(30,15$, and $7.5 \mathrm{mg})$ was prepared by sonication. Catalyst solution was spin coated onto $\mathrm{Nb} / \mathrm{SiO}_{2} / \mathrm{Si}$ substrate followed by drying at room temperature and baking at $160^{\circ} \mathrm{C}$ for $5 \mathrm{~min}$ in Ar atmosphere. The prepared substrates were placed in the CVD system and heated to $700^{\circ} \mathrm{C}$ in $\mathrm{Ar}$ atmosphere. Ar was replaced by $\mathrm{C}_{2} \mathrm{H}_{4}$ after the temperature was stabilized at $700^{\circ} \mathrm{C}$ and gas flow was maintained for $30 \mathrm{~min}$. The CVD system was finally cooled in $\mathrm{Ar}$ atmosphere for $30 \mathrm{~min}$.

The role of $\mathrm{Al}_{2} \mathrm{O}_{3}$ in the catalyst solution was (i) to support $\mathrm{Mo} / \mathrm{Fe}$ during the growth process (catalyst are well distributed on the comugated $\mathrm{Al}_{2} \mathrm{O}_{3}$ ) and (ii) to control density of CNTs. Vertical alignment of nanotubes, grown by thermal CVD, is achieved by controlling their density. ${ }^{12}$ In this work it was observed from a series of experiments that the density of thin MWCNTs $\left(\mathrm{cm}^{-2}\right)$ was controlled by varying $\mathrm{Al}_{2} \mathrm{O}_{3}$ content in the catalyst solution. The growth of 


\title{
Multistage field enhancement of tungsten oxide nanowires and its field emission in various vacuum conditions
}

\author{
Raghunandan Seelaboyina ${ }^{1}$, Jun Huang ${ }^{1}$, Jucheol Park ${ }^{2}$, \\ Dong Hun $\mathrm{Kang}^{2}$ and Won Bong Choi ${ }^{1,3}$ \\ ${ }^{1}$ Nanomaterials and Devices Lab, Department of Mechanical and Materials Engineering. \\ Florida International University, Miami, FL. 33174, USA \\ 2 Samsung Advanced Institute of Technology, Gibueng-Eup Young-Si Gyecnggi-Da, 449-712, \\ Korea \\ E-mail: chciw $\odot$ fiu.edu
}

Received 13 June 2006, in final form 13 August 2006

Published 7 September 2006

Online at stacks.iop.org/ $/ \mathrm{Nano} / 17 / 4840$

\begin{abstract}
We report on the field emission properties of tungsten oxide nanowires grown on a tungsten tip and its emission performance in various vacuum conditions. Tungsten oxide $\left(\mathrm{W}_{18} \mathrm{O}_{49}\right)$ nanowires were grown by thermal chemical vapour deposition in a mixture of $\mathrm{CH}_{4}$ and $\mathrm{H}_{2}$ on an electrochemically sharpened tungsten tip. The field emission measurements showed a low threshold field of $\sim 0.95 \mathrm{~V} \mu \mathrm{m}^{-1}$, high emission current of $170 \mu \mathrm{A}$ and a lange field enhancement factor of $\sim 19800$. High emission current of a few $\mu$ A was also observed in relatively poor vacuum at $3 \times 10^{-3} \mathrm{Torr}$, and the emission properties were recoverable at $1 \times 10^{-6}$ Torr.
\end{abstract}

(Some figures in this article are in colour only in the electronic version)

\section{Introduction}

To date, field emitters based on various nanomaterials have been considered for several vacuum microelectronic applications These emitters utilize local field enhancement at the apex of each nanoscale protrusion or micro-tip to lower the threshold voltage for field emission. They have been demonstrated in various applications such as field emission displays, microwave sources and many more [1-3]. However, their emission property is degraded ineversibly in low vacuum and oxygen environments because of enhanced surface reactions such as ion back sputtering, physical etching and so on [4-6]. Ion back sputtering and physical etching especially at the emitter tip surface degrades the local geometry and can lead to irreversible damage of the emitters. Oxide materials are chemically inert, robust and have a relatively high melting temperature which leads to their stability in oxygen and low vacuum conditions i.e. $10^{-4}-10^{-5}$ Torr. So, field emitters based on oxide materials have been reported as alternative candidates for stable field emission in low vacuum conditions [7-9].

${ }^{3}$ Author to whom any correspondence should be addressed.
Among the oxide emitters, tungsten oxide is widely used in various applications such as high pressure discharge lamps, electron guns and scanning tunnelling microscopy tips. Tungsten oxide has also been demonstrated to be suitable for various other applications such as electrochromic, optochromic and gasochromic coatings for smart windows, information display and various sensors $[10,11]$. Tungsten oxide is an n-type semiconductor with a work function in the range of $5.59-5.7 \mathrm{eV}[12,13]$ which makes it attractive for the stated applications. Since the first report on tungsten oxide by Fumio ef al in 1971 [14], several other researchers have synthesized tungsten oxide nanowires [15-19]. Some of these synthesis methods include thermal treatment of tungsten films [16]. infrared irradiation heating of tungsten foils [17] and a themal oxidation approach [19]. Recently, Zhou et al reported the synthesis of tungsten oxide nano-tip arrays by two-step, high temperature, catalyst-free, physical vapour deposition [18]. Although field emission properties of tungsten oxide nanowires have been studied, to the best of our knowledge there are no reports on the multistage field enhancement effect and their emission performance in various vacuum conditions. In this study we have synthesized a two-stage field emitter in an attempt to maximize the emitter performance such as field 


\title{
Recent Progress of Carbon Nanotube Field Emitters and Their Application
}

\author{
Raghunandan Seelaboyina and Wonbong Choi*
}

Nanomaterials and Device Laboratory, Department of Mechanical and Materiais Engineering. Florida Intenational University. Miami, FL 33174, USA

Received: Fobnuary 8, 2007; Accepted: March 8, 2007; Revised: May 23, 2007

Abstract: The potential of utilizing carbon vanotube field emission properties is an atractive feature for future vacuum electronic devices including: high power microwave, miniature $\mathrm{x}$-ray, backlight for liquid crystal displays and flat panel displays. Their high emission current, bno scale geometry. chemical inertuess and low threshold voltage for emission are attractive features for the field emission applications. In this paper we review the recent developments of carbon unotube field emitters and their device applications. We also discuss the hatest results on field emission current amplification achieved with an electron multipler microchannel plate, and emission perfornance of nultistage field emitter based on oxide nmowire operated poor vacum

Keywords: Carbon nanotubes, field emission, field emitters, vacuum microelectronics, nanowires, miniature $\mathrm{x}$-ray, field emission display microchannel plate, microwave, emission unifomity, vacuum stability, tungsten oxide, multistage, chemical vapor deposition, field enbancement.

\section{INTRODUCTION}

Carbon nanotube (CNT) has been envisioned as one of the promising materials for field emitters, because of their bigh aspect ratio $(\sim 1000)$ and atomically sharp apex, which enhances local field and lowers threshold field for electron emission $(\sim 0.5-1 \mathrm{~V} / \mu \mathrm{m})$ [1]. They also possess high electrical and thermal conductivity and high chemical and temperature stability all these unique properties make CNT a robust emitter with high emission stability [2]. Field emission (FE) is a quantum mechanical phenomenon in which electrons tunnel from a condensed phase into vacuum under application of high electro static field. Quantitative description of the process is given Fowler-Nordheim (FN) theory which gives the relation between emission current and the applied electric field [3]. FE from CNTs was first reported for an individual multiwall carbon nanotube (MWNT) by Rinzler ot al [1]. In later years the mechanism of electron emission from CNTs was confimed to be FE process [4]. It has been well documented that CNTs have better FE properties than other sharp metal based emitters. Some of the drawbacks with metal based field emitters are bigh current fluctuations, high fabrication cost and limited life time owing to field-induced sharpening and ion sputtering, which leads to destruction of the emitters. CNT is robust and bighly crystalline covalent structure which is less susceptible to surface migration of carbon atoms because of their strong $\mathrm{C}-\mathrm{C}$ covalent bonding [5] Apart from these unique properties, vertically aligned CNTs (Fig. la) and tower array stmuctures that are vital for vacuum microelectronic applications have been demonstrated [6-9]. The vertically oriented high aspect ratio $\mathrm{CNT}$ structures will provide high field enhancement and low threshold voltage for electron emission. Ever since the first report on CNT FE there have been several reports on various devices. Some of the most promising ones are field emission displays (FED) [10], electron guns for next generation scanning electron microscopes (SEM) and transmission electron microscopes (TEM), backlight for liquid crystal displays (LCD) [11], miniature X-ray [12] and source for high powered nicrowave (HPM) devices [13]. Although there has been significaut advancement in CNT based FE devices, there still remain some obstacles in realizing the devices. In this paper, we provide a brief review on the recent developments and patents on CNT based FE devices.

- Address correspondence to this author at the Nanomaterials and Device Laboratory, Department of Mechanical and Materials Engineering. Florida Laboratory, Departuent of Mechanical and Matenials Engineening, Flonda
Interuational University, Minmi, FL 33174, USA; Tel: 1-305-348-1973; Fax: 1-305-348-1932; E-mail: choiw afinedu

$1872-2105 / 07 \$ 100.00+.00$

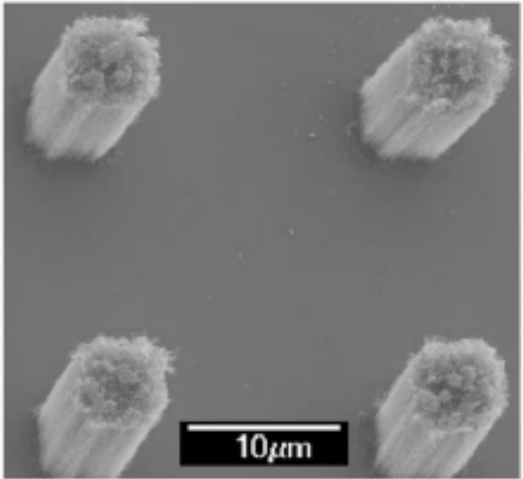

(a)

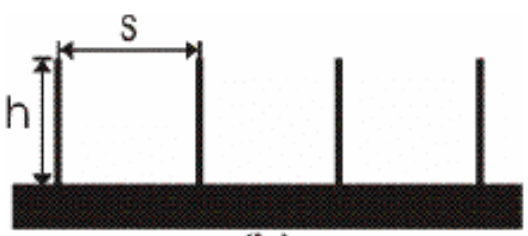

(b)

Fig. (1). (a) Aligned CNT towers (b) Schematic of CNT-CNT distance to minimize screening effect, where $h, 5$ are height and distance [16]

\section{CARBON NANOTUBE AS FIELD EMITTER}

A carbon nanotube consists of either one cylindrical graphene sheet, single-wall nanotube (SWNT) or of several nested cylinders with an interlayer spacing of $0.34-0.36 \mathrm{~mm}$, multi-wall carbon nanotube (MWNT). SWNTs and MWNTs lengths are usually well over $1 \mu \mathrm{m}$ with diameters ranging from $1 \mathrm{~nm}$ for $S W N T$ s to $\sim 30 \mathrm{~nm}$ for MWNTs. Nanotubes are usually closed at both ends by fullerene-like half spheres that contain both pentagons and hexagons [2]. Because of their large aspect ratio $(\sim 1000)$ and small tip radius, electron emission can be achieved at low electric fields due to bigher field concentration. A single CNT is capable of

○ 2007 Bentham Science Publishers Ltd. 


\title{
Catalysts effect on single-walled carbon nanotube branching
}

\author{
Jun Huang ${ }^{\text {a }}$, Do Hyun Kim ", Raghunandan Seelaboyina " , Banglore K. Rao ${ }^{\text {, }}$, Dake Wang ${ }^{\text {, }}$, \\ Minseo Park ${ }^{\circ}$, WonBong Choi ${ }^{2, *}$

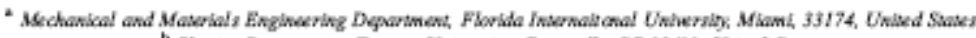

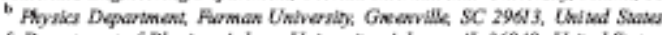

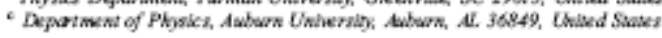 \\ Received 6 Apeil 2006; received in revised foem 29 Speom ter 2006 ; accoped 14 December 2006 \\ Avulable celine 8 Jaury 2007
}

Abstract

The catalytic effect cn the Yshaped singk-wallal carbon nanctubes (Y-SWNTs) branching is investignted. The formation of Y-shaped tranches is frund to be dependent on the catalysts composition, which can be correlated to the Gìbs free energy of metal carbide fomnation. Fasier cabide formex, like Mo or $\mathrm{Zr}$, have a strong tendency to attach to the sidewall of SWNTs, enhancing the degree of carbon namotube tranching. The electrical conductance of the Y-SWNTs demonstrated retificaticn chancteristics at room temperature, which is attrìuted to the Schot tky barrier fomned at the junction positicn. The Y-SWNTs can be used as a building blod for future nanodectronic, serser and composite. C 2007 Elsevier B.V. All rights reserved.

Keywords: Caboe nosoube; Catalyss; Yshapod

\section{Introduction}

Carbon nanotubes (CNTs) are among the promising candidates for next generation nanoelectronics because of the unique electrical properties and nanoscale size [1]. Among all the studies for CNTs, complex three-point nanotube junctions (Yand T-junctions) have been proposed as the building blocks for future nanoelectronics [2]. Y-shaped single-walled carbon nanotube (Y-SWNT) is a novel structure consisting of three terminals with different chirality which shows different electrical properties. Theoretical studies [3-5] predict the rectification behavior from symmetrical Y-SWNTs, implying that the switching behavior in nanoscale transistor can be expected by manipulating the third terminal of Y-SWNTs. In addition, the electrical characteristic of Y-SWNTs provides insights to the study of CNT networks (also known as CNT mats) which are expected to have immediate application in nanoelectronics and sensors [6].

For real applications, it is highly desirable to control the growth of Y-SWNTs. The synthesis of Y-shaped carbon nanotubes has been extensively studied by several groups.

\footnotetext{
- Correspoeding auther Tel $z+1305348$ 1973; fax: +1 3053481932

Emall address: choimafin odu (W. Cool).
}

Scuseria [3] and Chernozatonskii [4] proposed the first structural model of the Y-shaped carbon nanotubes by theoretical ealculations. The synthesis of Y-shaped carbon namotubes by chemical vapor deposition (CVD) method was reported by several groups $[7,8]$ by using an anodic ahuminum oxide (AAO) template to grow multi-walled carbon nanotubes (MWNTs). Since then, Y-shaped carbon nanotubes (MWNTs and SWNTs) have been synthesized by different techniques [5]. These techniques include AAO template-sssisted grow th [8], chemical vapor deposition (CVD) of products gener ated from a pyrolysis of metalbcenes, nanowelding of overlapping isolated namotubes using high intensity electron beam, thermal docomposition of $\mathrm{C}_{60}$ fullerene [9], using Ti-doped vapor catalyst [10] and using polystrene microspheres arrays to hold the catalysts for multiple junction formation [11], efc. Most of the experimental reports of the Y-shaped CNTs were based on the multi-walled carbon nanotubes. However, the Y-shaped MWNTs (Y-MWNTs) are metallic and transistor modulation was demonstrated only at low temperatures. Compared with $\mathrm{Y}$ MWNTs, Y-SWNTs are more practical for nanoscale electronic devices because of the possible ballistic switching and reliable transport properties at room temperature. Recently, we have also suocessfully synthesized Y-SWNTs using Mo-doped Fe catalyst by thermal CVD [12]. 


\title{
Enhanced field emission from aligned multistage carbon nanotube emitter arrays
}

Raghunandan Seelaboyina ${ }^{\mathrm{a}^{\wedge}}$, Srinivasarao Boddepalli ${ }^{\mathrm{a} / t}$, Kyungseok Noh $^{\mathrm{b}}$, Minhyon Jeon ${ }^{\mathrm{b}}$, Wonbong Choi ${ }^{\mathrm{a}^{*}}$

${ }^{a}$ Nanomaterials and Device Lab, Department of Mechanical and Materials Engineering, Florida International University, Miami, FL 33174, USA

${ }^{\mathrm{b}}$ School of Nano Engineering, Center for Nano Manufacturing, Inje University, Gimhae, GyungNam, South Korea 621-749

E-mail: choiw@fiu.edu

\begin{abstract}
In this work we report on the synthesis and field emission properties of carbon nanotube multistage emitter arrays grown on porous silicon by catalytic thermal chemical vapor deposition. The vertically oriented multistage array structures consisted of SWNT and thin-MWNT grown on MWNT, confirmed by TEM and Raman analysis. Higher field emission current $\sim 32$ times and low threshold field $\sim 1.5$ times were obtained for these structures in comparison to only MWNTs arrays. The enhanced field emission results for these multistage emitters are a consequence of higher field concentration, which is $\sim 3$ times more than MWNTs. This efficient emitter design with enhanced field emission properties can be used in future micro-vacuum electronic devices.
\end{abstract}

\section{Submitted (NANOTECHNOLOGY)}

${ }^{\mathrm{a} \#, \mathrm{a}{ }^{\wedge}}$ Both authors have equal contribution, experimental work was done by ${ }^{\mathrm{a} \#}$, most analysis was done by ${ }^{\mathrm{a}^{\wedge}}$ $\mathrm{a}^{*}$ To whom correspondence should be addressed 


\title{
Amplified Field Emission from MgO-coated Carbon nanotube Microchannel
}

\section{Plate}

Raghunandan Seelaboyina, ${ }^{a}$ Srinivasarao Bodepalli, ${ }^{a}$ Jucheol Park, ${ }^{b}$ Wonbong Choi ${ }^{a, 2}$

${ }^{a}$ Nanomaterials and Device Lab, Department of Mechanical and Materials Engineering, Florida International University, Miami, FL 33174, USA

${ }^{b}$ Samsung Advanced Institute of Technology, Gihueng-Eup Young-Si Gyeonggi-Do, Korea 449-712.

\begin{abstract}
We have utilized the extremely high secondary emission properties of magnesium oxide coated carbon nanotubes (CNT) for the fabrication of a novel microchannel plate (MCP). The MCP was characterized for electron multiplication by placing it between a nanotube emitter array cathode and an anode. In addition to enhancing the field emission by $\sim 18$ times the MCP also protects the nanotubes from irreversible damage during vacuum arcing. This unique system consisting of MCP and field emitters will provide a robust cold electron source operating at moderate power with enhanced life time.

\section{Submitted (NANOLETTERS)}

${ }^{2}$ Corresponding author email: choiw@fiu.edu

Phone:(305)348-1973, Fax:(305)348-1932 


\title{
Thin Multiwall Carbon Nanotube Field Emitters with Microchannel Plate for High Current Emission
}

Raghunandan Seelaboyina, Jun Huang, Won Bong Choi

Center for Nanomaterials and Devices, Department of Mechanical and Materials Engineering, Florida International University, Miami, FL 33174, USA, choiw@fiu.edu

\begin{abstract}
We report on a method to amplify the electron current of carbon nanotube field emitter cathode using an electron multiplier and synthesis of thin multiwall carbon nanotubes by thermal chemical vapor deposition. A commercial microchannel plate which is an array of microscopic electron multipliers was used for this purpose. A microchannel plate placed between the cathode and anode amplified the current 3 times and also acted as protection shield for nanotubes during vacuum arcing. The increase in current is attributed to the electron multiplication, and the increase in field enhancement factor from 9300 to $12800(37 \%)$ with microchannel plate. The thin multiwall carbon nanotubes were synthesized using $\mathrm{Mo} / \mathrm{Fe}$ catalyst solution. The inner and outer wall diameters of the nanotubes were in the range of $2.5-5 \mathrm{~nm}$ and $7-15 \mathrm{~nm}$ respectively, determined by Raman spectroscopy and transmission electron microscopy. The nanotubes had excellent field emission property which is attributed to their thin wall diameters $\sim 10 \mathrm{~nm}$, high aspect ratio of 800 and the resulting high field enhancement factor of 9300 . The emission current was stable with an average fluctuation of $2 \%$.
\end{abstract}

\section{INTRODUCTION}

Carbon nanotube field emitters have shown great promise as cathodes in many applications including field emission displays and high-power radio-frequency devices, [1-4]. Unique properties of carbon nanotubes (CNTs) include high current density [5], low threshold field for emission, instantaneous response to field variation, good on/off ratio, resistance to temperature fluctuations, high durability and power efficiency make them ideal candidate for many of these applications. Here we report a novel method of placing a microchannel plate, which is an array of microscopic electron multipliers on top of the CNT emitters. This new approach provides two benefits; it amplifies the electron current and protects CNTs from irreversible damage in the event of vacuum arcing. Microchannel plate (MCP) operates by avalanche multiplication of secondary electrons [6], secondary electrons are generated when incident electrons strike the channel walls of MCP. A voltage applied across the ends of the MCP creates a field which accelerates the secondary electrons along the channel generating more electrons leading to avalanche multiplication. Micro channel plates are used in many applications; one of them is in high efficiency field emission display as demonstrated by Lee et al. [7]. A commercial MCP made out of lead silicate glass obtained from Hamatsu was used for this study

We synthesized thin multiwall carbon nanotubes which have shown good field emission properties in comparison with single and multiwall carbon nanotubes. Singlewall carbon nanotubes (SWNTs) have high aspect ratio and small radius of curvature $(\sim 1 \mathrm{~nm})$; they have been reported to have large field enhancement factor, low threshold voltage, and high emission current [8]. However, the degradation of emission current with time is an obstacle to practical 
VITA

\section{RAGHUNANDAN SEELABOYINA}

\section{EDUCATION}

Florida International University, Miami, FL

- Doctoral Candidate in Mechanical Engineering, Dec 2007

- M.S. Material Science Engineering, Dec 2003

Sri Siddartha Institute of Technology, Tumkur, India

- B.S. Mechanical Engineering, Dec 2000

\section{PUBLICATIONS}

- Seelaboyina, R., Bodepalli. S., Park, J., and Choi, W. B.(2007), Amplified Field Emission from $\mathrm{MgO}$-coated Carbon nanotube Microchannel plate, Nanoletters (Submitted).

- Seelaboyina, R., Bodepalli, S., Park, J., Noh, K., Jeon, M., and Choi, W. B.(2007), Enhanced field emission from aligned multistage carbon nanotube emitter arrays, Nanotechnology (Accepted).

- Seelaboyina, R., and Choi, W. B.(2007), Recent Progress of Carbon Nanotube Field Emitters and Their Application, Recent Patents in nanotechnology, 1238.

- Zheng, F., Jones, W., Wu, W., and Seelaboyina, R. (2007), Nanoceramic Processing for High-Power Multi-channel Electron Multiplier in Low Temperature Cofire Ceramic (LTCC), Journal of microelectronics and electronic packaging,4 93.

- Huang, J., Kim, D.H., Seelaboyina, R., Rao, B.K., Wang, D., Park, M. and Choi, W.B.(2006), Catalysts effect on single-walled carbon nanotube branching, Diamond and related materials, 161524.

- Seelaboyina, R., Huang, J., Park, J., Kang, D.H., and Choi, W.B.(2006), Multistage field enhancement of tungsten oxide nanowires and its field emission in various vacuum conditions, Nanotechnology, 174840.

- Seelaboyina, R., Huang, J. and Choi, W.B.(2006), Enhanced field emission of thinmultiwall carbon nanotubes by electron multiplication from microchannel plate, Appl. Phys. Lett., 88194104 and Virtual Journal of Nanoscale Science and Technology, 1321.

- Ghose, S., Krisch, M., Oganov, A.R., Gulve, R.P., Seelaboyina, R., Yang, H. and Saxena, S.K.(2006), Lattice dynamics of $\mathrm{MgO}$ at high pressure: theory and experiment, Phys. Rev. Lett., 96035507.

- Seelaboyina, R., Huang, J. and Choi, W.B.(2006), Thin multiwall carbon nanotube field emitters with microchannel plate for high current emission, MRS Symposium Proceedings, (Progress in Semiconductor Materials V-Novel Materials and Electronic and Optoelectronic Applications), 271. 
- Seelaboyina, R., Phatak, N., Gulve, R.P, Leirmann, H.P. and Saxena, S.K.(2005), Thermal expansion of nanocrystalline titanium dioxide, zinc oxide, nickel oxide, Thermal Conductivity, 27647.

\section{PRESENTATIONS}

- Seelaboyina, R., Huang, J., Bodepalli, S., and Choi, W.B.(2007), 5th annual NanoMaterials for Defense Applications Symposium, Field Emitters and Electron Multiplier Microchannel Plate for High Power Applications, San Diego, CA (Poster).

- Seelaboyina, R., Huang, J. and Choi, W.B.(2007), Nanomaterials Symposium, Carbon nanotube emission enhancement by microchannel plate and field emission of tungsten oxide multistage field emitters, TMS Annual Meeting, Orlando, FL (Oral).

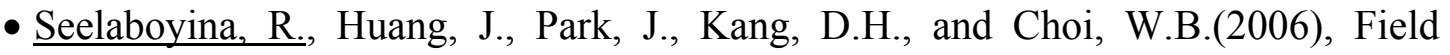
emission performance in various vacuum conditions and multistage field enhancement effect of tungsten oxide nanowires, AVS 53rd International Symposium and Exhibition, San Francisco, CA (Oral).

- Seelaboyina, R., Huang, J. and Choi, W.B.(2006), Amplification system for high current emission from thin-multiwall carbon nanotubes, ICNDST and ADC joint conference, Research triangle park, NC (Poster).

- Seelaboyina, R., Choi, Y.C., Huang, J., Saxena, S.K. and Choi, W.B.(2005), Synthesis and field emission properties of thin Multiwall Carbon nanotubes, SMEC, Miami, FL (Oral).

- Seelaboyina, R. and Saxena, S.K. (2004), Thermal Expansion of some refractory solids to melting temperatures, ITCC 28, Knoxville, TN (Oral).

- Seelaboyina, R., Leirmann, H.P. and Saxena, S.K.(2004), Thermal expansion of nano $\mathrm{TiO}_{2}$, ITES 16, Knoxville, TN (Poster).

AWARDS

- First place (2007), Material Advantage (ASM/TMS) technical oral competition, FIU, Miami FL.

- Third place (2007), Graduate student association (GSA) scholarly forum technical oral competition, FIU, Miami FL.

- Conference travel grants (2006) from FIU graduate school and AVS (Dorothy M. and Earl S. Hoffman travel grant) to attend AVS 53rd International Symposium and Exhibition, San Francisco, CA, November 12-17.

- First prize (2006), Material Advantage (ASM/TMS) poster competition, FIU, Miami FL. 